\title{
UNE INTERPRÉTATION MODULAIRE DE LA VARIÉTÉ TRIANGULINE
}

\author{
CHRISTOPHE BREUIL, EUGEN HELLMANN ET BENJAMIN SCHRAEN
}

\begin{abstract}
RÉSUMÉ. En utilisant le système de Taylor-Wiles-Kisin construit dans un travail récent de Caraiani, Emerton, Gee, Geraghty, Paškūnas et Shin, nous construisons un analogue de la variété de Hecke. Nous montrons que cette variété coïncide avec une union de composantes irréductibles de l'espace des représentations galoisiennes triangulines. Nous précisons les relations de cette construction avec les conjectures de modularité dans le cas cristallin ainsi qu'avec une conjecture de Breuil sur le socle des vecteurs localement analytiques de la cohomologie complétée. Nous donnons également une preuve d'une conjecture de Bellaïche et Chenevier sur l'anneau local complété en certains points des variétés de Hecke.

ABSTRACT. Using a patching module constructed in recent work of Caraiani, Emerton, Gee, Geraghty, Paškūnas and Shin we construct some kind of analogue of an eigenvariety. We can show that this patched eigenvariety agrees with a union of irreducible components of a space of trianguline Galois representations. Building on this we discuss the relation with the modularity conjectures for the crystalline case, a conjecture of Breuil on the locally analytic socle of representations occurring in completed cohomology and with a conjecture of Bellaïche and Chenevier on the complete local ring at certain points of eigenvarieties.
\end{abstract}

\section{TABLE DES MATIÈRES}

1. Introduction 2

2. Espaces de déformations $\quad 5$

2.1. Quelques définitions de géométrie analytique rigide 6

2.2. L'espace des représentations triangulines $\quad 7$

2.3. Espaces de déformations cristallines et poids de Sen 11

2.4. Espaces de formes automorphes 14

3. La variété de Hecke-Taylor-Wiles 17

3.1. Vecteurs localement $R$-analytiques 17

3.2. La variété de Hecke-Taylor-Wiles 19

3.3. Premières propriétés 21

3.4. Série principale localement $\mathbb{Q}_{p}$-analytique de $G_{p} \quad 25$

3.5. Densité des points classiques 26

3.6. Composantes irréductibles de $X_{p}(\bar{\rho}) \quad 33$

4. Application aux variétés de Hecke 35 
4.1. L'espace des représentations galoisiennes de pente finie

4.2. Un résultat de modularité infinitésimale

5. Appendice

5.1. Un peu d'analyse fonctionnelle non archimédienne

5.2. Vecteurs localement analytiques

5.3. Un lemme sur les systèmes de racines de type $A$

5.4. Quelques résultats de géométrie rigide

5.5. Descente de la lissité en géométrie rigide

\section{IntRoduCtion}

Soit $p>2$ un nombre premier. Cet article a pour but d'utiliser la méthode des systèmes de Taylor-Wiles telle que généralisée par Kisin, puis par Caraiani-Emerton-Gee-GeraghtyPaškūnas-Shin ([15]), pour étudier le problème des formes compagnons dans la théorie des formes automorphes $p$-adiques sur les groupes unitaires compacts à l'infini.

Considérons $F^{+}$un corps de nombres totalement réel, ainsi que $F$ une extension quadratique totalement imaginaire non ramifiée de $F^{+}$, et $G$ un groupe unitaire sur $F^{+}$déployé par $F$, compact à l'infini et isomorphe à $\mathrm{GL}_{n}$ au-dessus de toutes les places divisant $p$. Soit $L$ une extension finie de $\mathbb{Q}_{p}$. Si $U^{p}$ désigne un sous-groupe compact ouvert de $G\left(\mathbb{A}_{F^{+}}^{p \infty}\right)$, on désigne par $\widehat{S}\left(U^{p}, L\right)$ l'espace des fonctions continues $G\left(F^{+}\right) \backslash G\left(\mathbb{A}_{F^{+}}^{\infty}\right) / U^{p} \rightarrow L$. C'est un $L$-espace de Banach muni d'une action continue et unitaire du groupe $G\left(F^{+} \otimes_{\mathbb{Q}} \mathbb{Q}_{p}\right)$. On note $\widehat{S}\left(U^{p}, L\right)^{\text {an }} \subset \widehat{S}\left(U^{p}, L\right)$ le sous-espace des vecteurs localement analytiques pour l'action du groupe de Lie $p$-adique $G\left(F^{+} \otimes_{\mathbb{Q}} \mathbb{Q}_{p}\right)$. Si $\mathfrak{p}$ est un idéal maximal de la « grosse » algèbre de Hecke $\widehat{\mathbb{T}}\left(U^{p}, \mathcal{O}_{L}\right)\left[\frac{1}{p}\right]$, et $\delta$ un caractère continu du groupe $T\left(F^{+} \otimes_{\mathbb{Q}} \mathbb{Q}_{p}\right)$, on appelle forme surconvergente de poids $\delta$ associée à $\mathfrak{p}$ un vecteur propre, de caractère propre $\delta$, du monoïde d'Atkin-Lehner $T\left(F^{+} \otimes_{\mathbb{Q}} \mathbb{Q}_{p}\right)^{+}$agissant sur $\widehat{S}\left(U^{p}, L\right)[\mathfrak{p}]^{\text {an, } \mathrm{N}_{0}}$ où $N_{0}$ est un sous-groupe compact ouvert stable par $T\left(F^{+} \otimes_{\mathbb{Q}} \mathbb{Q}_{p}\right)^{+}$du groupe des matrices unipotentes supérieures $N\left(F^{+} \otimes_{\mathbb{Q}} \mathbb{Q}_{p}\right)$. Soit $\rho$ la représentation $p$-adique, de dimension $n$, du groupe de Galois absolu de $F$ associée à l'idéal p. Dans [11], l'un d'entre nous (C. B.) donne une description conjecturale des caractères $\delta$ pour lesquels il existe des formes surconvergentes associées à $\mathfrak{p}$ dans le cas où toutes les représentations $\rho_{\mathcal{G}_{F_{\tilde{v}}}}$ obtenues par restriction aux groupes de décomposition au-dessus de $p$ sont potentiellement cristallines et « génériques ». Cette conjecture a été par la suite étendue par Hansen ([32]) au cas où certaines $\left.\rho\right|_{\mathcal{G}_{\tilde{v}}}$ peuvent être triangulines.

Un des résultats de cet article est que l'existence des formes surconvergentes associées à tous les $\delta$ décrits dans [11] dans le cas cristallin est en fait une conséquence des conjectures de modularité dans le cas cristallin. 
Désignons par $\bar{\rho}$ la réduction de $\rho$ modulo l'idéal maximal de $\mathcal{O}_{L}$. La recherche des caractères $\delta$ associés à un idéal $\mathfrak{p}$ comme ci-dessus, ou de façon équivalente, à la représentation $\rho$ équivaut à déterminer les points de la $\bar{\rho}$-composante $\mathcal{E}\left(U^{p}\right)_{\bar{\rho}}$ de la variété de Hecke de niveau modéré $U^{p}$ (voir par exemple [18]) sur lesquels la (pseudo-)représentation canonique est isomorphe à $\rho$. De tels points sont appelés points compagnons. La variété de Hecke $\mathcal{E}\left(U^{p}\right)_{\bar{\rho}}$ est une sous-variété rigide analytique fermée du produit $\mathfrak{X}_{\bar{\rho}, S} \times \widehat{T}\left(F^{+} \otimes_{\mathbb{Q}} \mathbb{Q}_{p}\right)$ où $\mathfrak{X}_{\bar{\rho}, S}$ est un espace analytique rigide sur $L$ paramétrant certains relevés de $\bar{\rho}$ en caractéristique 0 et $\widehat{T}\left(F^{+} \otimes_{\mathbb{Q}} \mathbb{Q}_{p}\right)$ est l'espace analytique rigide paramétrant les caractères continus du groupe $T\left(F^{+} \otimes_{\mathbb{Q}} \mathbb{Q}_{p}\right)$. La variété $\mathcal{E}\left(U^{p}\right)_{\bar{\rho}}$ est ainsi munie d'une application poids $\omega: \mathcal{E}\left(U^{p}\right)_{\bar{\rho}} \rightarrow \widehat{T}\left(\mathcal{O}_{F^{+}} \otimes_{\mathbb{Z}} \mathbb{Z}_{p}\right)$. Le but de cet article est de construire, au moyen de systèmes de Taylor-Wiles-Kisin de niveau infini en $p$ construits dans [15], un espace analytique rigide $X_{p}(\bar{\rho})$ sur $L$ muni d'une application poids $\omega_{\infty}: X_{p}(\bar{\rho}) \rightarrow \widehat{T}\left(\mathcal{O}_{F}+\otimes_{\mathbb{Z}} \mathbb{Z}_{p}\right) \times \mathfrak{X}_{S_{\infty}}$, où $\mathfrak{X}_{S_{\infty}}$ est isomorphe à un produit de boules unité ouvertes, et un diagramme commutatif cartésien dans la catégorie des espaces analytiques rigides réduits

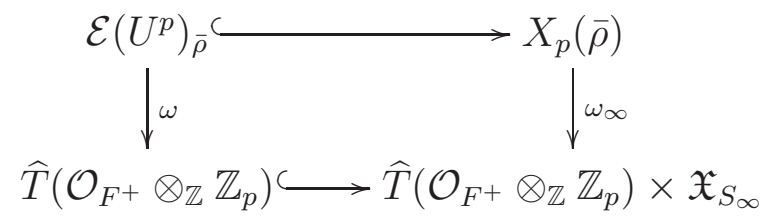

où la flèche horizontale du bas provient de l'inclusion d'un point fermé dans $\mathfrak{X}_{S_{\infty}}$. Par construction, l'espace rigide analytique $X_{p}(\bar{\rho})$ est un sous-espace analytique fermé de l'espace produit

$$
\prod_{v \mid p}\left(\mathfrak{X}_{\bar{\rho}_{\tilde{v}}}^{\square} \times \widehat{T}\left(F_{v}^{+}\right)\right) \times \mathbb{U}^{g}
$$

où $\mathfrak{X}_{\bar{\rho}_{\tilde{v}}}^{\square}$ désigne l'espace des relèvements (cadrés) de la représentation résiduelle $\bar{\rho}_{\tilde{v}}=\left.\bar{\rho}\right|_{\mathcal{G}_{F_{\tilde{v}}}}$ et $\mathbb{U}^{g}$ est un certain produit de boules unités ouvertes. Il est en fait nécessaire, dans tout ce qui précède de supposer $p>2 n+1$ et $\left.\bar{\rho}\right|_{\mathcal{G}_{F\left(\zeta_{p}\right)}}$ absolument irréductible dans l'utilisation des résultats de [15].

Si $v \mid p$ notons $X_{\text {tri }}^{\square}\left(\bar{\rho}_{\tilde{v}}\right)$ le plus petit sous-espace analytique fermé du produit $\mathfrak{X}_{\bar{\rho}_{\tilde{v}}}^{\square} \times \widehat{T}\left(F_{v}^{+}\right)$ contenant tous les couples $(r, \delta)$ où $r$ est un relèvement (cadré) triangulin de $\bar{\rho}_{\tilde{v}}$ et $\delta$ un paramètre de $r$. Cet espace a été introduit dans un cadre un peu différent par l'un d'entre nous (E. H.) dans [33] suivant une idée de Kisin ([41]). Nous prouvons alors le résultat suivant (Théorème 3.20 et Corollaire 3.19) :

Théorème 1.1. L'espace $X_{p}(\bar{\rho})$ est réduit et s'identifie à une union de composantes irréductibles de $\prod_{v \mid p} X_{\text {tri }}^{\square}\left(\bar{\rho}_{\tilde{v}}\right) \times \mathbb{U}^{g}$.

Au passage nous prouvons également le résultat suivant sur la structure de l'espace $X_{\text {tri }}^{\square}\left(\bar{\rho}_{\tilde{v}}\right)$ (Théorème 2.6).

Théorème 1.2. L'espace $X_{\text {tri }}^{\square}\left(\bar{\rho}_{\tilde{v}}\right)$ est équidimensionnel de dimension $n^{2}+\left[F_{v}^{+}: \mathbb{Q}_{p}\right] \frac{n(n+1)}{2}$.

Nous renvoyons le lecteur à l'énoncé du Théorème 2.6 pour des propriétés plus fines de l'espace $X_{\text {tri }}^{\square}(\bar{\rho})$. La preuve de ce résultat s'appuie essentiellement sur les travaux de Kedlaya, Pottharst et Xiao ([39]).

Nous introduisons alors la notion de composante irréductible automorphe de l'espace $\prod_{v \mid p} X_{\text {tri }}^{\square}\left(\bar{\rho}_{\tilde{v}}\right)$, il s'agit d'une composante irréductible $X$ de ce produit telle que $X \times \mathbb{U}^{g}$ apparaisse dans $X_{p}(\bar{\rho})$. Il nous semble alors naturel de conjecturer (Conjecture 3.22) : 
Conjecture 1.3. Une composante irréductible de $\prod_{v \mid p} X_{\mathrm{tri}}^{\square}\left(\bar{\rho}_{\tilde{v}}\right)$ est une composante irréductible automorphe si et seulement si son ouvert de lissité (plus précisément son intersection avec l'ouvert $\prod_{v \mid p} U_{\mathrm{tri}}^{\square}\left(\bar{\rho}_{\tilde{v}}\right)^{\mathrm{reg}}$ de $\prod_{v \mid p} X_{\mathrm{tri}}^{\square}\left(\bar{\rho}_{\tilde{v}}\right)$ défini dans la section 2) contient un point cristallin.

Nous prouvons alors le résultat suivant (Propositions 3.26 et 3.27) :

Théorème 1.4. La Conjecture 1.3 est équivalente aux conjectures de modularité dans le cas cristallin (plus précisément à la Conjecture 3.24).

Nous prouvons par ailleurs que cette conjecture implique une partie d'une conjecture de l'un d'entre nous (C. B.) concernant le module de Jacquet-Emerton des vecteurs localement analytiques de la cohomologie complétée (cf. Corollaire 4.4).

Dans la dernière partie, nous utilisons cette construction pour prouver un grand nombre de cas d'une conjecture de Bellaïche et Chenevier sur la structure infinitésimale en certains points de la variété de Hecke $Y$ d'un groupe unitaire $G$. On peut voir cette conjecture comme une conjecture $R=T$ infinitésimale. Nous renvoyons le lecteur au corps du texte pour tout ce qui concerne les notations et conventions relatives à ces variétés de Hecke.

Théorème 1.5. Supposons $p>2 n+1$ et $\left.\bar{\rho}\right|_{\mathcal{G}_{F\left(\zeta_{p}\right)}}$ absolument irréductible. Soit $z=(\pi, \chi)$ un point de la variété de Hecke $\mathcal{E}\left(U^{p}\right)_{\bar{\rho}}$ correspondant à une représentation automorphe $\pi$ de $G(\mathbb{A})$. Supposons qu'en restriction à tout groupe de décomposition au-dessus de $p$ la représentation galoisienne $\rho_{\pi}$ associée à $\pi$ soit cristalline et que les valeurs propres de son Frobenius cristallin $\varphi$ soient deux-à-deux distinctes. Sous ces hypothèses, le caractère $\chi$ définit un drapeau de $D_{\text {cris }}\left(\rho_{\pi}\right)$ stable par $\varphi$ que nous supposons être en position générale relativement à la filtration de de Rham. Alors l'anneau local complété $\hat{\mathcal{O}}_{\mathcal{E}\left(U^{p}\right)_{\bar{\rho}}, z}$ pro-représente le foncteur des déformations triangulines de $\rho$ munies d'une triangulation déformant la triangulation définie par le drapeau $\varphi$-stable de $D_{\text {cris }}\left(\rho_{\pi}\right)$.

Le plan de l'article est le suivant. Nous commençons dans la section 2 par définir les espaces locaux $X_{\text {tri }}^{\square}(\bar{r})$ pour $\bar{r}$ une représentation continue du groupe de Galois d'une extension finie de $\mathbb{Q}_{p}$ sur un espace vectoriel de dimension finie et de caractéristique $p$. Nous prouvons alors le Théorème 1.2 et donnons un certain nombre de résultats additionnels sur les points cristallins qui apparaissent dans cet espace. La section 3.2 contient la construction de l'espace $X_{p}(\bar{\rho})$ ainsi que la preuve de ses propriétés géométriques. Les Théorèmes 1.1 et 1.4, font l'objet de la section 3.6. La section 4 fait le lien avec les variétés de Hecke. Nous y prouvons le caractère cartésien du diagramme 1.1 ainsi que le Théorème 1.5 .

Remerciements : Nous remercions Ahmed Abbes pour avoir répondu à nos questions. Une partie de cet article a été écrite lors de différents séjours dans les institutions suivantes : I.H.É.S., Math. Institut de l'Université de Bonn et M.S.R.I. En particulier B. S. souhaite remercier l'I.H.É.S. pour son hospitalité prolongée lors de la rédaction de cet article, ainsi que le B.I.C.M.R. de Pékin. Par ailleurs, C. B. et B. S. sont membres du projet ANR ThéHopaD (ANR-2011-BS01-005), et E. H. est membre du SFB -TR 45 de la DFG.

Notations : On fixe un nombre premier $p$, une extension algébrique $\overline{\mathbb{Q}}_{p}$ de $\mathbb{Q}_{p}$, ainsi qu'un isomorphisme entre $\overline{\mathbb{Q}}_{p}$ et $\mathbb{C}$.

Si $L$ est un corps local non archimédien localement compact, on note $|\cdot|_{L}$ sa valeur absolue normalisée, c'est-à-dire telle que la norme d'une uniformisante de $L$ soit l'inverse 
du cardinal du corps résiduel de $L$. On note $|\cdot|_{p}$ la valeur absolue sur $\overline{\mathbb{Q}}_{p}$ telle que $|p|_{p}=p^{-1}\left(\right.$ donc $|\cdot|_{L}=|\cdot|_{p}^{\left[L: \mathbb{Q}_{p}\right]}$ sur $\left.L\right)$.

Si $K$ est un corps, on note $\mathcal{G}_{K}=\operatorname{Gal}(\bar{K} / K)$ le groupe de Galois absolu de $K$. Lorsque $K$ est de plus une extension finie de $\mathbb{Q}_{p}$, on note $\operatorname{rec}_{K}: K^{\times} \rightarrow \mathcal{G}_{K}^{\text {ab }}$ le morphisme de réciprocité de la théorie du corps de classes normalisé de telle sorte que l'image d'une uniformisante soit un élément de Frobenius géométrique. On note $\varepsilon$ le caractère $p$-adique cyclotomique de $\mathcal{G}_{K}$, de sorte que $\varepsilon \circ \operatorname{rec}_{K}$ est le caractère $x \mapsto N_{K / \mathbb{Q}_{p}}(x)|x|_{K}$ de $K^{\times}$. On adopte comme convention que le poids de Hodge-Tate de $\varepsilon$ est 1.

Si un groupe algébrique est désigné par une lettre majuscule romaine $(G, H, N, T, \ldots)$ on désigne par la lettre minuscule gothique correspondante $(\mathfrak{g}, \mathfrak{h}, \mathfrak{n}, \mathfrak{t}, \ldots)$ son algèbre de Lie.

Fixons brièvement quelques conventions concernant le groupe $\mathrm{GL}_{n}$. On note $B$ le sousgroupe de Borel des matrices triangulaires supérieures, et $T$ son sous-tore maximal constitué des matrices diagonales. Ce choix détermine un ensemble $\Delta=\left\{\alpha_{1}, \cdots, \alpha_{n-1}\right\}$ de racines simples, $\alpha_{i}$ désignant le caractère algébrique $\left(t_{1}, \cdots, t_{n}\right) \mapsto t_{i} t_{i+1}^{-1}$. Pour $1 \leq i \leq n$, on note $\beta_{i}$ le cocaractère $t \mapsto(\underbrace{t, \cdots, t}_{i}, 1, \cdots, 1)$, de telle sorte que l'on ait $\alpha_{i} \circ \beta_{j}(t)=t^{\delta_{i, j}}$. Nous convenons que la notion de dominance pour un caractère du tore $T$ ou de son algèbre de Lie $\mathfrak{t}$ est toujours relative à ce choix de racines simples. Si $I \subset\{1, \cdots, n-1\}$, on note $P_{I}$ le sous-groupe parabolique contenant $B$ correspondant à l'ensemble $\left\{\alpha_{i} \mid i \in I\right\}$, et $\bar{P}_{I}$ le sous-groupe parabolique opposé, on désigne par $L_{I}$ leur sous-groupe de Levi commun et $W_{I} \subseteq W$ son groupe de Weyl. Rappelons que si $M$ désigne un module de plus haut poids de $U\left(\mathfrak{g l}_{n}\right)$, relativement à l'algèbre de Lie $\mathfrak{b}$, le sous-groupe $P_{I}$ est dit adapté à $M$ si l'action de $\mathfrak{p}_{I}$ sur $M$ se prolonge en une représentation algébrique de $P_{I}$. Si $\rho$ désigne la demi-somme des racines positives, $\lambda$ un caractère de $\mathfrak{t}$ et $w$ un élément du groupe de Weyl de $G$, on définit $w \cdot \lambda=w(\lambda+\rho)-\rho$. Si $\lambda$ est un poids dominant entier de $\mathfrak{t}$, le $U(\mathfrak{g})$-module simple de plus haut poids $w \cdot \lambda$ est adapté à $\mathfrak{p}_{I}$, si et seulement si $w \cdot \lambda$ est un poids dominant de $L_{I}$, ce qui est encore équivalent à ce que $w \in{ }^{I} W$ l'ensemble des représentants de $W_{I} \backslash W$ de longueur minimale ([37, § 9.4]).

Soit $L$ et $K$ deux extensions finies de $\mathbb{Q}_{p}$, on note $K_{0}$ l'extension maximale non ramifiée sur $\mathbb{Q}_{p}$ contenue dans $K$ et $K_{0}^{\prime}$ l'extension maximale non ramifiée sur $\mathbb{Q}_{p}$ contenue dans $K\left(\left\{\zeta_{p^{n}} \mid n \geq 1\right\}\right)$ où $\zeta_{p^{n}}$ est un système compatible de racines $p^{n}$-ièmes de l'unité. On note de plus $\mathcal{R}_{K}$ l'anneau de Robba de $K$ et $\mathcal{R}_{L, K}=L \otimes_{\mathbb{Q}_{p}} \mathcal{R}_{K}$. On rappelle que $\mathcal{R}_{K}$ est l'anneau des séries formelles $f=\sum_{n \in \mathbb{Z}} a_{n} z^{n}$ avec $a_{n} \in K_{0}^{\prime}$ convergeant sur une couronne $r(f) \leq|z|_{p}<1([39, \S 2.2])$.

Si $H$ est un groupe de Lie $p$-adique abélien, on note $\widehat{H}$ l'espace analytique rigide sur $\mathbb{Q}_{p}$ représentant le foncteur associant à un espace affinoïde $X=\operatorname{Sp}(A)$ le groupe $\operatorname{Hom}_{\text {cont }}\left(H, A^{\times}\right)$des morphismes de groupes continus $H \rightarrow A^{\times}$(voir par exemple $[25, \S$ $6.4]$ ). Si $X$ est un espace analytique rigide sur $\mathbb{Q}_{p}$ et $L$ une extension finie de $\mathbb{Q}_{p}$, on note $X_{L}=\operatorname{Sp}(L) \times_{\operatorname{Sp}\left(\mathbb{Q}_{p}\right)} X$.

\section{Espaces DE DÉFormations}

Soit $L$ une extension finie de $\mathbb{Q}_{p}$ dans $\overline{\mathbb{Q}}_{p}$. On note $\mathcal{O}_{L}$ son anneau d'entiers et $k_{L}$ son corps résiduel. On fixe $\varpi_{L}$ une uniformisante de $\mathcal{O}_{L}$. 
2.1. Quelques définitions de géométrie analytique rigide. Soit $X$ un espace analytique rigide sur $L$. On appelle fermé analytique de $X$ un "analytic subset" au sens de $[9, \S 9.5 .2]$ (appelé aussi "analytic set" dans [24]).

Définition 2.1. Une partie $U \subset X$ est un ouvert de Zariski si son complémentaire est un fermé analytique de $X$.

Un ouvert de Zariski est en particulier un ouvert admissible que l'on munit de la structure d'espace analytique rigide sur $L$ induite par $X$.

Définition 2.2. (i) Une partie $Z$ de $X$ est dense au sens de Zariski dans $X$, ou Zariskidense dans $X$, si le plus petit fermé analytique de $X$ contenant $Z$ est l'espace sous-jacent $\grave{a} X$.

(ii) Si $A$ et $B$ sont deux parties de $X$, on dit que $A$ s'accumule en $B$ si pour tout $b \in B$ et pour tout voisinage affinoïde $U$ de $b$, il existe un voisinage affinoïde $V \subset U$ de $b$ tel que $A \cap V$ est Zariski-dense dans $V$. Un ensemble de $X$ est dit d'accumulation s'il s'accumule en lui-même (cf. [18, p. 979]).

Les composantes irréductibles d'un espace analytique rigide sont définies en [24, Def. 2.2.2]. On vérifie facilement en utilisant [24, Lem. 2.2.3] que, si $X$ est un espace analytique rigide irréductible et $U \subset X$ un ouvert de Zariski non vide de $X$, l'espace analytique rigide $U$ est connexe.

Soit $\mathrm{FS}_{\mathcal{O}_{L}}$ la catégorie des schémas formels $\mathcal{X}$ sur $\mathcal{O}_{L}$, localement noethériens et dont la réduction modulo un idéal de définition est un schéma localement de type fini sur $k_{L}$. On note $\mathcal{X} \mapsto \mathcal{X}^{\text {rig }}$ le foncteur construit par Raynaud et Berthelot de la catégorie $\mathrm{FS}_{\mathcal{O}_{L}}$ vers la catégorie des espaces analytiques rigides sur $L$ (cf. [38, § 7], [8, § 0.2]).

Si $R$ est une $\mathcal{O}_{L}$-algèbre locale, plate, complète, noethérienne et de corps résiduel fini $\left(\operatorname{sur} k_{L}\right)$, de sorte que le schéma formel $\operatorname{Spf}(R)$ est un objet de $\mathrm{FS}_{\mathcal{O}_{L}}$, il existe une bijection canonique entre l'ensemble des composantes irréductibles du schéma $\operatorname{Spec}\left(R\left[\frac{1}{p}\right]\right)$ et l'ensemble des composantes irréductibles de l'espace analytique rigide $\operatorname{Spf}(R)^{\text {rig }}$ définie de la façon suivante. Une composante irréductible de $\operatorname{Spec}\left(R\left[\frac{1}{p}\right]\right)$ correspond à un idéal premier minimal $\mathfrak{p}$ de $R\left[\frac{1}{p}\right]$. On obtient alors une composante irréductible de $\operatorname{Spf}(R)^{\text {rig }}$ en considérant l'image du morphisme $\operatorname{Spf}(R /(\mathfrak{p} \cap R))^{\text {rig }} \rightarrow \operatorname{Spf}(R)^{\text {rig }}$. Le caractère bijectif de cette application est une conséquence de [24, Thm. 2.3.1] et du fait que les idéaux premiers minimaux de $R$ sont en bijection avec les composantes connexes de la normalisation de $\operatorname{Spf}(R)$.

Si $R$ est une $\mathcal{O}_{L}$-algèbre comme ci-dessus, mais supposée de plus normale, par [38, Prop. 7.3.6] le morphisme $R\left[\frac{1}{p}\right] \rightarrow \mathcal{O}\left(\operatorname{Spf}(R)^{\text {rig }}\right)$ identifie l'anneau $R\left[\frac{1}{p}\right]$ à l'anneau des fonctions bornées sur $\operatorname{Spf}(R)^{\text {rig }}$.

Proposition 2.3. Si $X$ est un espace analytique rigide réduit, l'ensemble des points lisses de $X([9, \S 7.3 .2])$ est un ouvert de Zariski de $X$ qui est de plus Zariski-dense dans $X$.

Un espace analytique rigide $X$ est dit équidimensionnel de dimension $d$ si pour tout point $x \in X$, l'anneau local complété $\widehat{\mathcal{O}}_{X, x}$ de $X$ en $x$ est un anneau local équidimensionnel de dimension $d$. De même $X$ est dit sans composante immergée si pour tout point $x \in X$, tous les idéaux premiers associés de $\widehat{\mathcal{O}}_{X, x}$ sont des idéaux premiers minimaux.

Pour $f: X \rightarrow Y$ un morphisme entre espaces analytiques rigides et $x \in X$, on dit que $f$ est étale en $x$ si la condition de [36, Déf. 1.7.10 i)] est vérifiée. 
Si $X$ est un espace analytique rigide réduit, on note $\mathcal{O}_{X}^{+}$le sous-faisceau de $\mathcal{O}_{X}$ des fonctions de norme plus petite que 1 .

2.2. L'espace des représentations triangulines. Nous rappelons ici la construction de l'espace des déformations triangulines d'une représentation galoisienne en caractéristique $p$.

Fixons $K$ une extension finie de $\mathbb{Q}_{p}$. On suppose que l'extension $L$ de $\mathbb{Q}_{p}$ est suffisamment grande pour qu'il existe $\left[K: \mathbb{Q}_{p}\right]$ plongements de $K$ dans $L$, on note $\operatorname{Hom}(K, L)$ l'ensemble de ces plongements et $\mathcal{T}=\widehat{K^{\times}}$(l'espace analytique rigide sur $\mathbb{Q}_{p}$ paramétrant les caractères continus de $\left.K^{\times}\right)$. Si $\mathbf{k}=\left(k_{\tau}\right)_{\tau \in \operatorname{Hom}(K, L)} \in \mathbb{Z}^{\operatorname{Hom}(K, L)}$, on note $x^{\mathbf{k}}$ l'élément de $\mathcal{T}(L)$ défini par $x \mapsto \prod_{\tau \in \operatorname{Hom}(K, L)} \tau(x)^{k_{\tau}}$. Soit $n$ un entier strictement positif. Pour $\mathbf{k}=\left(k_{\tau, i}\right)_{1 \leq i \leq n, \tau: K \hookrightarrow L} \in\left(\mathbb{Z}^{n}\right)^{\operatorname{Hom}(K, L)}$, on note $\delta_{\mathbf{k}}$ l'élément de $\mathcal{T}^{n}(L)$ défini par $\left(x_{1}, \cdots, x_{n}\right) \mapsto \prod_{i, \tau} \tau\left(x_{i}\right)^{k_{\tau, i}}$ (où $\left(x_{1}, \cdots, x_{n}\right) \in\left(K^{\times}\right)^{n}$ ) et on s'autorise l'abus de langage consistant à désigner par le même signe sa restriction à $\left(\mathcal{O}_{K}^{\times}\right)^{n}$. Un tel poids est dit dominant si, pour tout $\tau: K \hookrightarrow L$, on a $k_{\tau, 1} \geq \cdots \geq k_{\tau, n}$. Si de plus, pour tout $\tau \in \operatorname{Hom}(K, L)$, on a $k_{\tau, 1}>\cdots>k_{\tau, n}$, on dit que le poids est strictement dominant.

Rappelons que, si $L^{\prime}$ désigne une extension finie de $\mathbb{Q}_{p}$, les éléments de $\mathcal{T}\left(L^{\prime}\right)$ sont canoniquement en bijection avec l'ensemble des classes d'isomorphisme de $\left(\varphi, \Gamma_{K}\right)$-modules de rang 1 sur $\mathcal{R}_{L^{\prime}, K}$ par la construction $\delta \mapsto \mathcal{R}_{L^{\prime}, K}(\delta)$ de [39, Cons. 6.2.4].

Soit $\bar{r}$ une représentation continue de $\mathcal{G}_{K}$ sur un $k_{L^{-}}$espace vectoriel $V_{k_{L}}$ de dimension $n$. Fixons une fois pour toute une base $\bar{b}$ de $V_{k_{L}}$, ce qui nous permet de définir la $\mathcal{O}_{L^{-}}$ algèbre locale noethérienne complète $R_{\bar{r}}^{\square}$ qui pro-représente le foncteur associant à une $\mathcal{O}_{L}$-algèbre artinienne locale $A$ de corps résiduel $k_{L}$ l'ensemble des classes d'équivalence de triplets $\left(V_{A}, \iota, b\right)$ où $V_{A}$ est un $A$-module libre de rang $n$ muni d'une action continue $A$-linéaire de $\mathcal{G}_{K}, \iota$ un isomorphisme $\mathcal{G}_{K}$-équivariant entre $V_{A} \otimes_{A} k_{L}$ et $V_{k_{L}}$, et $b$ une base de $V_{A}$ se réduisant sur $\bar{b}$ à travers $\iota$. On note $\mathfrak{X}_{\bar{r}}^{\square}$ l'espace analytique rigide sur $L$ associé au schéma formel $\operatorname{Spf}\left(R_{\bar{r}}^{\square}\right)$. On note $\mathcal{T}_{\text {reg }}$ le complémentaire dans $\mathcal{T}_{L}=\mathcal{T} \times_{\mathbb{Q}_{p}} L$ de l'ensemble des $L$-points $x \mapsto x^{-\mathbf{i}}$ et $x \mapsto x^{\mathbf{1 + i}}|x|_{K}$ pour $\mathbf{i} \in \mathbb{Z}_{\geq 0}^{\operatorname{Hom}(K, L)}$ et $\mathcal{T}_{\text {reg }}^{n} \subset \mathcal{T}_{L}^{n}$ l'ouvert de Zariski des points $\left(\delta_{1}, \cdots, \delta_{n}\right)$ tels que $\delta_{i} \delta_{j}^{-1} \in \mathcal{T}_{\text {reg }}$ pour $i \neq j$. On désigne par $U_{\text {tri }}^{\square}(\bar{r})^{\text {reg }}$ l'ensemble des points $(x, \delta) \in \mathfrak{X}_{\bar{r}}^{\square} \times \mathcal{T}_{\text {reg }}^{n}$ tels que, si $r_{x}$ désigne la représentation de $\mathcal{G}_{K}$ associée à $x$, son $\left(\varphi, \Gamma_{K}\right)$-module $D_{\text {rig }}^{\dagger}\left(r_{x}\right)$ est triangulin (voir [22], [4, § 2], [39, § 6]) et $\delta=\left(\delta_{1}, \cdots, \delta_{n}\right)$ est un système de paramètres de $r_{x}$ (ou plus simplement un paramètre de $\left.r_{x}\right)$, ce qui signifie qu'il existe une filtration croissante de $D_{\text {rig }}^{\dagger}\left(r_{x}\right)$ par des sous- $\mathcal{R}_{k(x), K^{-}}$ modules stable par $\varphi$ et $\Gamma_{K}$ (nécessairement facteurs directs comme $\mathcal{R}_{k(x), K^{-m o d u l e s}}$ ) dont les gradués successifs sont isomorphes à $\mathcal{R}_{k(x), K}\left(\delta_{1}\right), \cdots, \mathcal{R}_{k(x), K}\left(\delta_{n}\right)$ comme $\left(\varphi, \Gamma_{K}\right)$ modules, où $k(x)$ est le corps résiduel de $\mathfrak{X}_{\bar{r}}^{\square}$ au point $x$.

Définition 2.4. On appelle espace des déformations triangulines de $\bar{r}$, et on le note $X_{\mathrm{tri}}^{\square}(\bar{r})$, l'espace analytique rigide sur L obtenu comme adhérence de Zariski de $U_{\mathrm{tri}}^{\square}(\bar{r})^{\text {reg }}$ dans $\mathfrak{X}_{\bar{r}}^{\square} \times \mathcal{T}_{L}^{n}$, i.e. le fermé analytique intersection des fermés analytiques de $\mathfrak{X}_{\bar{r}}^{\square} \times \mathcal{T}_{L}^{n}$ contenant $U_{\mathrm{tri}}^{\square}(\bar{r})^{\mathrm{reg}}$ muni de sa structure de variété analytique rigide réduite $([9, \S 9.5 .3$ Prop. 4]).

Remarquons que la variété analytique rigide $X_{\text {tri }}^{\square}(\bar{r})$ est séparée car fermée dans la variété analytique rigide séparée $\mathfrak{X}_{\bar{r}}^{\square} \times \mathcal{T}_{L}^{n}$ (cf. [9, p. 393]).

Notons $\mathcal{W}=\widehat{\mathcal{O}_{K}^{\times}}$(l'espace analytique rigide sur $\mathbb{Q}_{p}$ paramétrant les caractères continus de $\left.\mathcal{O}_{K}^{\times}\right)$et $\mathcal{W}_{L}=\mathcal{W} \times_{\mathbb{Q}_{p}} L$. La restriction d'un caractère de $K^{\times}$à $\mathcal{O}_{K}^{\times}$induit un morphisme 
d'espaces analytiques rigides $\mathcal{T} \rightarrow \mathcal{W}$ sur $\mathbb{Q}_{p}$. On note $\omega^{\prime}$ le morphisme $X_{\text {tri }}^{\square}(\bar{r}) \rightarrow \mathcal{T}_{L}^{n}$ obtenu en composant l'inclusion de $X_{\text {tri }}^{\square}(\bar{r})$ dans $\mathfrak{X}_{\bar{r}}^{\square} \times \mathcal{T}_{L}^{n}$ avec la seconde projection, on obtient une application poids $\omega: X_{\text {tri }}^{\square}(\bar{r}) \rightarrow \mathcal{W}_{L}^{n}$ en composant $\omega^{\prime}$ avec le morphisme de restriction $\mathcal{T}_{L}^{n} \rightarrow \mathcal{W}_{L}^{n}$.

Remarque 2.5. Si on note $X_{\text {tri }}^{\square}(\bar{r})^{\text {reg }}$ l'image réciproque de $\mathcal{T}_{\text {reg }}^{n}$ par $\omega$, l'espace $X_{\text {tri }}^{\square}(\bar{r})^{\text {reg }}$ est un ouvert de Zariski de $X_{\mathrm{tri}}^{\square}(\bar{r})$. On a $U_{\mathrm{tri}}^{\square}(\bar{r})^{\mathrm{reg}} \subset X_{\mathrm{tri}}^{\square}(\bar{r})^{\mathrm{reg}}$ mais cette inclusion est toujours stricte. Il existe en effet des points $\left(r_{x}, \delta_{x}\right)$ de $X_{\text {tri }}^{\square}(\bar{r})$ tels que $\delta_{x}$ ne soit pas un paramètre de la représentation $r_{x}$, même si $r_{x}$ est trianguline et $\delta_{x}$ est régulier. Le Théorème 6.3.13 de [39] montre cependant que $r_{x}$ est toujours trianguline.

Le reste de cette section est presque entièrement consacré à la preuve du résultat suivant concernant la géométrie de l'espace $X_{\text {tri }}^{\square}(\bar{r})$.

Théorème 2.6. ( $i)$ L'espace $X_{\mathrm{tri}}^{\square}(\bar{r})$ est équidimensionnel de dimension $n^{2}+\left[K: \mathbb{Q}_{p}\right] \frac{n(n+1)}{2}$. (ii) L'ensemble $U_{\mathrm{tri}}^{\square}(\bar{r})^{\mathrm{reg}}$ est un ouvert de Zariski de $X_{\mathrm{tri}}^{\square}(\bar{r})$ qui est Zariski-dense dans $X_{\text {tri }}^{\square}(\bar{r})$.

(iii) L'ouvert $U_{\text {tri }}^{\square}(\bar{r})^{\text {reg }}$ est lisse sur $\mathbb{Q}_{p}$ et la restriction de l'application $\omega^{\prime}$ à $U_{\text {tri }}^{\square}(\bar{r})^{\text {reg }}$ est un morphisme lisse.

Démonstration. La stratégie de la preuve est la suivante. Nous allons construire un espace analytique rigide lisse $\mathcal{S}^{\square}(\bar{r})$ s'insérant dans un diagramme commutatif comme ci-dessous

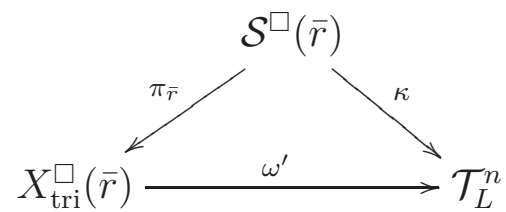

où le morphisme $\pi_{\bar{r}}$ est lisse de dimension relative $n$ et d'image $U_{\text {tri }}^{\square}(\bar{r})^{\text {reg }}$ et le morphisme $\kappa$ est lisse de dimension relative $n^{2}+\left[K: \mathbb{Q}_{p}\right] \frac{n(n-1)}{2}$.

L'espace analytique rigide $\mathcal{S}^{\square}(\bar{r})$ représente le foncteur

$$
X \mapsto\left\{\left(r, \mathrm{~F}_{\bullet}, \delta, \nu\right)\right\} / \sim
$$

où $X$ est un espace analytique réduit sur $L$ et $r$ est un morphisme continu $\mathcal{G}_{K} \rightarrow \mathrm{GL}_{n}\left(\mathcal{O}_{X}^{+}\right)$ tel qu'en tout point $x \in X$, la réduction de $r \otimes \mathcal{O}_{k(x)}$ coïncide avec $\bar{r}$. De plus $\mathrm{F}$ • est une filtration croissante de $\mathbf{D}_{\text {rig }}^{\dagger}(r)$ par des sous- $\left(\varphi, \Gamma_{K}\right)$-modules localement sur $X$ facteurs directs comme $\mathcal{R}_{X}$-modules tels que $F_{0}=0$ et $F_{n}=\mathbf{D}_{\text {rig }}^{\dagger}(r)$ et $\delta=\left(\delta_{1}, \ldots, \delta_{n}\right) \in \mathcal{T}_{\text {reg }}^{n}(X)$. Finalement $\nu=\left(\nu_{1}, \ldots, \nu_{n}\right)$ est une collection de trivialisations $\nu_{i}: \operatorname{gr}_{i}(D) \cong \mathcal{R}_{X}\left(\delta_{i}\right)$ (avec les notations de [35, §2]). La flèche $\kappa$ correspond alors au morphisme de foncteurs $\left(r, \mathrm{~F}_{\bullet}, \delta, \nu\right) \mapsto \delta$

Pour prouver la représentabilité de ce foncteur, nous suivons la stratégie de [19], [33] et $[35]$.

Soit $\mathcal{S}_{n}$ l'espace analytique rigide sur $L$ représentant le foncteur

$$
X \longmapsto\left\{\left(D, \operatorname{Fil}_{\bullet}(D), \delta, \nu\right)\right\} / \sim
$$

où $X$ est comme avant, $D$ est un $\left(\varphi, \Gamma_{K}\right)$-module sur $\mathcal{R}_{X}$ de rang $n$, et Fil• est une filtration croissante de $D$ par des sous- $\left(\varphi, \Gamma_{K}\right)$-modules localement sur $X$ facteurs directs comme $\mathcal{R}_{X}$-modules tels que $F_{0}=0$ et $F_{n}=D$. De plus $\delta=\left(\delta_{1}, \ldots, \delta_{n}\right) \in \mathcal{T}_{\text {reg }}^{n}(X)$ et $\nu=\left(\nu_{1}, \ldots, \nu_{n}\right)$ est une collection de trivialisations $\nu_{i}: \operatorname{gr}_{i}(D) \cong \mathcal{R}_{X}\left(\delta_{i}\right)$. Ce foncteur est représentable par un espace analytique rigide séparé d'après [35, Theorem 2.4]. Soit 
$\mathcal{S}_{n}^{\text {adm }} \subset \mathcal{S}_{n}$ le sous-ensemble admissible au sens de [34, Theorem 1.2], i.e. le sous-espace ouvert adique $^{1}$ maximal de $\mathcal{S}_{n}$ tel qu'il existe un fibré vectoriel $\mathcal{V}$ sur $\mathcal{S}_{n}^{\text {adm }}$ et un morphisme continu $\mathcal{G}_{K} \rightarrow \operatorname{Aut}_{\mathcal{O}_{\mathcal{S}_{n}}^{\text {adm }}}(\mathcal{V})$ tels que $\mathbf{D}_{\text {rig }}^{\dagger}(\mathcal{V})$ est l'image inverse du $\left(\varphi, \Gamma_{K}\right)$-module universel de $\mathcal{S}_{n}$ sur $\mathcal{S}_{n}^{\text {adm }}$. Cet ouvert est non vide car toute représentation cristalline à poids de Hodge-Tate deux-à-deux distincts et Frobenius suffisamment générique permet d'en construire un point.

Soit $\pi: \mathcal{S}_{n}^{\square, \text { adm }} \rightarrow \mathcal{S}_{n}^{\text {adm }}$ le $\mathrm{GL}_{n}$-torseur des trivialisations du fibré vectoriel $\mathcal{V}$. Il existe donc un isomorphisme $\beta: \pi^{*} \mathcal{V} \simeq \mathcal{O}_{\mathcal{S}_{n}^{\square, a d m}}^{n}$ sur $\mathcal{S}_{n}^{\square, \text { adm }}$ et l'action de $\mathcal{G}_{K}$ sur $\mathcal{V}$ est donnée par un morphisme continu

$$
\tilde{r}: \mathcal{G}_{K} \rightarrow \mathrm{GL}_{n}\left(\Gamma\left(\mathcal{S}_{n}^{\square, \mathrm{adm}}, \mathcal{O}_{\mathcal{S}_{n}^{\square, \mathrm{adm}}}\right)\right) .
$$

Comme $\mathcal{G}_{K}$ est topologiquement engendré par un nombre fini d'éléments (voir par exemple [59, Prop. II.14 et I.25 ]), l'ensemble des points $x$ de $\mathcal{S}_{n}^{\square, \text { adm }}$ où $\tilde{r}$ se factorise à travers

$$
\mathrm{GL}_{n}\left(\Gamma\left(\mathcal{S}_{n}^{\square, \mathrm{adm}}, \mathcal{O}_{\mathcal{S}_{n}^{\square, \mathrm{adm}}}^{+}\right)\right) \subset \mathrm{GL}_{n}\left(\Gamma\left(\mathcal{S}_{n}^{\square, \mathrm{adm}}, \mathcal{O}_{\mathcal{S}_{n}^{\square, \mathrm{adm}}}\right)\right)
$$

est un ouvert admissible de $\mathcal{S}_{n}^{\square \text {,adm }}$. Comme de plus $\mathcal{G}_{K}$ est compact, cet ouvert est non vide. Rappelons, que l'on a fixé une représentation résiduelle $\bar{r}: \mathcal{G}_{K} \rightarrow \mathrm{GL}_{n}\left(k_{L}\right)$. On définit alors $\mathcal{S}^{\square}(\bar{r}) \subset \mathcal{S}^{\square \text {,adm }}$ comme l'ouvert admissible non vide (en considérant des relevés cristallins de $\bar{r}$ ) des points $x$ où le morphisme $\tilde{r}_{x}$ se réduit sur $\bar{r}$ modulo l'idéal maximal de $k(x)^{+}$. Il est alors immédiat de vérifier que $\mathcal{S}^{\square}(\bar{r})$ représente bien le foncteur (2.2). Notons $r_{X}: \mathcal{G}_{K} \rightarrow \mathrm{GL}_{n}\left(\mathcal{O}_{\mathcal{S}^{\square}(\bar{r})}^{+}\right)$le morphisme universel sur $\mathcal{S}^{\square}(\bar{r})$.

On définit alors le morphisme $\kappa: \mathcal{S}^{\square}(\bar{r}) \rightarrow \mathcal{T}_{L}^{n}$ comme la composée du morphisme $\mathcal{S}^{\square}(\bar{r}) \hookrightarrow \mathcal{S}_{n}^{\square, \text { adm }} \rightarrow \mathcal{S}_{n}^{\text {adm }} \hookrightarrow \mathcal{S}_{n}$, qui est lisse de dimension relative $n^{2}$, et de la projection canonique $\mathcal{S}_{n} \rightarrow \mathcal{T}_{L}^{n}$, qui est lisse de dimension relative $\left[K: \mathbb{Q}_{p}\right] \frac{n(n-1)}{2}$ par [35, Theorem 2.4(i)]. Comme $\mathcal{T}_{L}$ est lisse de dimension $n\left[K: \mathbb{Q}_{p}\right]+n$, on en conclut que $\mathcal{S}^{\square}(\bar{r})$ est lisse et équidimensionnel de dimension $n^{2}+n+\frac{n(n+1)}{2}\left[K: \mathbb{Q}_{p}\right]$. On a également un morphisme $\mathcal{S}^{\square}(\bar{r}) \rightarrow \mathfrak{X}_{\bar{r}}^{\square} \times \mathcal{T}_{L}^{n}$ donné par $\left(r, \mathrm{~F}_{\bullet}, \delta, \nu\right) \mapsto(r, \delta)$. La description des points de $\mathcal{S}^{\square}(\bar{r})$ montre que l'image de ce morphisme est exactement $U_{\text {tri }}^{\square}(\bar{r})^{\text {reg }}$. On obtient ainsi un morphisme

d'image $U_{\text {tri }}^{\square}(\bar{r})^{\text {reg }}$.

$$
\pi_{\bar{r}}: \mathcal{S}^{\square}(\bar{r}) \longrightarrow X_{\text {tri }}^{\square}(\bar{r})
$$

Montrons tout d'abord que l'espace $X_{\text {tri }}^{\square}(\bar{r})$ est équidimensionnel, de dimension

$$
\operatorname{dim} X_{\text {tri }}^{\square}(\bar{r})=n^{2}+\left[K: \mathbb{Q}_{p}\right] \frac{n(n+1)}{2} .
$$

D'après le Lemme 2.12 de [35], les points de $U_{\text {tri }}^{\square}(\bar{r})^{\text {reg }}$ sont strictement triangulins au sens de [39, Def. 6.3.1]. Le Corollaire 6.3.10 de loc. cit. permet alors de construire un morphisme projectif et birationnel, donc surjectif, $f: \tilde{X}_{\text {tri }}^{\square}(\bar{r}) \rightarrow X_{\text {tri }}^{\square}(\bar{r})$ et, si $D:=\mathbf{D}_{\text {rig }}^{\dagger}\left(f^{*} r_{X}\right)$, une filtration Fil. $(D) \subset D$ de $D$ par des sous- $(\varphi, \Gamma)$-modules de $D$ ainsi que des suites exactes courtes pour $1 \leq i \leq n$

$$
0 \longrightarrow \operatorname{gr}_{i} D \longrightarrow \mathcal{R}_{\tilde{X}_{\mathrm{tri}}(\bar{r})}\left(\delta_{i}\right) \otimes \mathcal{L}_{i} \longrightarrow \mathcal{M}_{i} \longrightarrow 0
$$

où $\mathcal{L}_{i}$ est un fibré en droites sur $\tilde{X}_{\text {tri }}^{\square}(\bar{r})$ et $\mathcal{M}_{i}$ un $\mathcal{R}_{\tilde{X}_{\text {tri }}^{\square}(\bar{r})}$-module qui est (localement sur $\left.\tilde{X}_{\text {tri }}^{\square}(\bar{r})\right)$ tué par une puissance de $t$ et de support un fermé analytique $Z_{i} \subset \tilde{X}_{\text {tri }}^{\square}(\bar{r})$

1. Nous utilisons ici le fait qu'un ouvert de l'espace adique associé à un espace analytique rigide quasiséparé est quasi-séparé et donc provient lui-même d'un espace analytique rigide d'après [36, §1.1.11]. 
ne contenant aucune composante irréductible de $\tilde{X}_{\text {tri }}^{\square}(\bar{r})$. On a de plus $f^{-1}\left(U_{\text {tri }}^{\square}(\bar{r})^{\text {reg }}\right) \subset$ $\tilde{X}_{\text {tri }}^{\square}(\bar{r}) \backslash \bigcup_{i} Z_{i}$.

Soit $U \subset \tilde{X}_{\text {tri }}^{\square}(\bar{r})$ l'intersection du complémentaire de $\bigcup_{i} Z_{i}$ dans $\tilde{X}_{\text {tri }}^{\square}(\bar{r})$ avec l'image réciproque de $\mathcal{T}_{\text {reg }}^{n} \subset \mathcal{T}_{L}^{n}$ via $\tilde{X}_{\text {tri }}^{\square}(\bar{r}) \rightarrow X_{\text {tri }}^{\square}(\bar{r}) \rightarrow \mathcal{T}_{L}^{n}$. Il s'agit d'un ouvert de Zariski de $\tilde{X}_{\text {tri }}^{\square}(\bar{r})$ qui contient $f^{-1}\left(U_{\text {tri }}^{\square}(\bar{r})^{\text {reg }}\right)$, ce qui prouve qu'il est aussi Zariski dense dans $\tilde{X}_{\text {tri }}^{\square}(\bar{r})$. Notons $U^{\square} \rightarrow U$ le $\mathbb{G}_{m}^{n}$-torseur des trivialisations des fibrés en droites $\mathcal{L}_{i}$. L'espace $U^{\square}$ possède par construction la propriété suivante. Il existe des trivialisations canoniques des images inverses des faisceaux $\mathcal{L}_{i}$ sur $U^{\square}$ telles que, si $g: Y \rightarrow U$ est un morphisme, et $s_{i}$ des trivialisations $\mathcal{O}_{Y} \simeq g^{*}\left(\mathcal{L}_{i}\right)$, il existe un unique morphisme $Y \rightarrow U^{\square}$ relevant $g$ de sorte que les $s_{i}$ sont les images inverses des trivialisations canoniques sur $U^{\square}$. La propriété universelle de $\mathcal{S}^{\square}(\bar{r})$ nous permet par ailleurs de construire un morphisme $s: U^{\square} \rightarrow \mathcal{S}^{\square}(\bar{r})$ tel que $\pi_{\bar{r}} \circ s$ est la composée

$$
U^{\square} \longrightarrow U \stackrel{\left.f\right|_{U}}{\longrightarrow} X_{\mathrm{tri}}^{\square}(\bar{r}) .
$$

Comme $f$ est la composée d'éclatements et de normalisations, on peut trouver un ouvert de Zariski et Zariski dense $V$ de $X_{\text {tri }}^{\square}(\bar{r})$ tel que $f^{-1}(V) \subset U$ et $f$ induise un isomorphisme de $f^{-1}(V)$ sur $V$. Notons de même $V^{\square} \rightarrow f^{-1}(V)$ le $\mathbb{G}_{m}^{n}$-torseur des trivialisations des faisceaux $\left.\mathcal{L}_{i}\right|_{f^{-1}(V)}$, il est facile de vérifier que $V^{\square}$ n'est autre que l'image réciproque de $f^{-1}(V)$ dans $U^{\square}$. La propriété universelle de $V^{\square} \rightarrow V$ permet de construire un morphisme $\pi_{\bar{r}}^{\square}$ de $\pi_{\bar{r}}^{-1}(V)$ dans $V^{\square}$ dont la composée avec $V^{\square} \rightarrow f^{-1}(V)$ est $\left.f^{-1}\right|_{V} \circ \pi_{\bar{r}}$. On a alors $\left.\pi_{\bar{r}}^{\square} \circ s\right|_{V \square}=\operatorname{Id}_{V \square}$. Or, la description des points de $\mathcal{S}^{\square}(\bar{r})$ montre que le morphisme $\pi_{\bar{r}}^{\square}$ de $\pi_{\bar{r}}^{-1}(V)$ dans $V^{\square}$ est injectif, on en conclut que l'on a donc également $s \circ \pi_{\bar{r}}^{\square}=\operatorname{Id}_{\pi_{\bar{r}}^{-1}(V)}$, ce qui implique, puisque tous les espaces sont réduits, que $\pi_{\bar{r}}^{\square}$ induit un isomorphisme de $\pi_{\bar{r}}^{-1}(V)$ sur $V^{\square}$. Ainsi, $V^{\square}$ est équidimensionnel de dimension $n^{2}+n+\frac{n(n+1)}{2}\left[K: \mathbb{Q}_{p}\right]$, $V^{\square} \rightarrow V$ étant un $\mathbb{G}_{m}^{n}$-torseur, on en conclut que l'ouvert $V$ est équidimensionnel de dimension $n^{2}+\left[K: \mathbb{Q}_{p}\right] \frac{n(n+1)}{2}$. Comme $V$ est un ouvert dense de $X_{\text {tri }}^{\square}(\bar{r})$, il en est de même de $X_{\text {tri }}^{\square}(\bar{r})$, ceci prouve le point (i).

Prouvons à présent que l'application $\pi_{\bar{r}}$ de $\mathcal{S}^{\square}(\bar{r})$ vers $X_{\text {tri }}^{\square}(\bar{r})$ est lisse de dimension relative $n$. Pour ce faire, il suffit de prouver que si $x \in \mathcal{S}^{\square}(\bar{r})$ et $y=\left(r_{y}, \delta_{y}\right)=\pi_{\bar{r}}(x) \in$ $U_{\text {tri }}^{\square}(\bar{r})^{\text {reg }}$, il existe un isomorphisme d'anneaux locaux complets

$$
\widehat{\mathcal{O}}_{\mathcal{S}^{\square}(\bar{r}), x} \simeq \widehat{\mathcal{O}}_{X_{\mathrm{tri}}^{\square}(\bar{r}), y} \llbracket x_{1}, \ldots, x_{n} \rrbracket .
$$

Notons $A=\widehat{\mathcal{O}}_{X_{\text {tri }}^{\square}(\bar{r}), y}$ et $B=\widehat{\mathcal{O}}_{\mathcal{S} \square(\bar{r}), x}$ dans ce qui suit, de sorte que $\pi_{\bar{r}}$ induise un morphisme local d'anneaux locaux complets $A \rightarrow B$.

Le morphisme de projection $X_{\text {tri }}^{\square}(\bar{r}) \rightarrow \mathfrak{X}_{\bar{r}}^{\square}$ donne lieu à un morphisme d'anneaux locaux complets

$$
\widehat{\mathcal{O}}_{\mathfrak{X}_{\bar{r}}^{\square}, r_{y}} \longrightarrow \widehat{\mathcal{O}}_{X_{\text {tri }}^{\square}(\bar{r}), y} .
$$

En utilisant [43, Lem. 2.3.3 et Prop. 2.3.5], on voit qu'il existe de plus un isomorphisme topologique entre $\widehat{\mathcal{O}}_{\mathfrak{X} \overline{\bar{r}}, r_{y}}$ et $R_{r_{y}}^{\square}$, l'anneau de déformation universel cadré de $r_{y}$, la représentation galoisienne correspondant au point $y$. Soit $\mathcal{F}$ l'unique triangulation du $\left(\varphi, \Gamma_{K}\right)$ module $D$ de $r_{y}$ correspondant au point $y$ et $R_{r_{y}, \mathcal{F}}^{\square}$ l'anneau de déformation universel cadré de la paire $\left(r_{y}, \mathcal{F}\right)$ en un sens évident. Comme précédemment, l'anneau $B=\widehat{\mathcal{O}}_{\mathcal{S} \square(\bar{r}), x}$ est naturellement isomorphe à une $R_{r_{y}, \mathcal{F}^{-}}^{\square}$ algèbre locale complète lisse de dimension relative $n$. Fixons donc $\left(x_{1}, \ldots, x_{n}\right)$ une famille de générateurs topologiques de la $R_{r_{y}, \mathcal{F}^{-}}^{\square}$ algèbre $B$. On définit alors une application continue $A$-linéaire de $A \llbracket X_{1}, \ldots, X_{n} \rrbracket$ dans $B$ envoyant 
$X_{i}$ sur $x_{i}$. Comme l'application $R_{r_{y}}^{\square} \rightarrow B$ se factorise à travers l'anneau $A$, on obtient au final une suite de morphismes

$$
R_{r_{y}}^{\square} \llbracket X_{1}, \ldots, X_{n} \rrbracket \rightarrow A \llbracket X_{1}, \ldots, X_{n} \rrbracket \rightarrow B .
$$

D'après [4, Prop. 2.3.6 et 2.3.9], l'application naturelle $R_{r_{y}}^{\square} \rightarrow R_{r_{y}, \mathcal{F}}^{\square}$ est surjective. Comme l'anneau $B$ est engendré par l'image de $R_{r_{y}}^{\square}$ et par les $x_{1}, \ldots, x_{n}$, on en conclut que l'application $A \llbracket X_{1}, \ldots, X_{n} \rrbracket \rightarrow B$ est surjective. Ces deux anneaux locaux étant noethériens, complets et de même dimension, il suffit de prouver que $A \llbracket X_{1}, \ldots, X_{n} \rrbracket$ est intègre pour conclure que $A \llbracket X_{1}, \ldots, X_{n} \rrbracket \rightarrow B$ est un isomorphisme. Il suffit donc de prouver que l'anneau $A$ est intègre. Comme $X_{\text {tri }}^{\square}(\bar{r})$ est réduit, on déduit de $[9, \S 7.2$,Prop. 8] que $A$ est réduit. Il suffit donc de prouver que cet anneau n'a qu'un seul idéal premier minimal. Pour cela, il suffit de prouver que la fibre du normalisé $X_{\text {tri }}^{\square}(\bar{r})^{\prime}$ de $X_{\text {tri }}^{\square}(\bar{r})$ au dessus de $y$ est un singleton. Remarquons que par la propriété universelle du normalisé, le morphisme $\pi_{\bar{r}}$ se factorise de façon unique à travers $X_{\text {tri }}^{\square}(\bar{r})^{\prime}$ puisque $\mathcal{S}^{\square}(\bar{r})$ est lisse donc normal. Comme $\tilde{X}_{\text {tri }}^{\square}(\bar{r})$ est normal, il en est de même de $U$ et donc de $U^{\square}$. Ainsi le morphisme $U^{\square} \rightarrow X_{\text {tri }}^{\square}(\bar{r})$ se factorise de façon unique à travers $X_{\text {tri }}^{\square}(\bar{r})^{\prime}$, de sorte que le diagramme ci-dessous est commutatif.

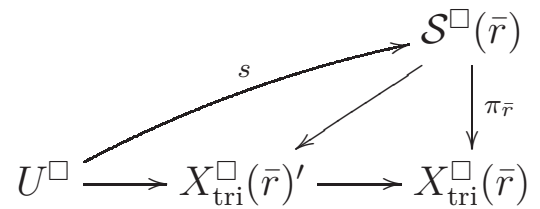

Puisque $f^{-1}\left(U_{\mathrm{tri}}^{\square}(\bar{r})^{\text {reg }}\right) \subset U$, l'existence de $s$ montre que tous les points de $X_{\mathrm{tri}}^{\square}(\bar{r})^{\prime}$ au dessus de $y \in U_{\text {tri }}^{\square}(\bar{r})^{\text {reg }}$ sont dans l'image de $\mathcal{S}^{\square}(\bar{r})$ dans $X_{\text {tri }}^{\square}(\bar{r})^{\prime}$. Comme la fibre de $\pi_{\bar{r}}$ au-dessus de $y$ est connexe (car isomorphe à $\mathbb{G}_{m}^{n}$ puisqu'il y a seulement une triangulation de $r_{y}$ de paramètre $\delta_{y}$ ), son image dans la fibre de $X_{\text {tri }}^{\square}(\bar{r})^{\prime}$ au dessus de $y$ doit également être connexe. Comme cette fibre est finie, il s'agit d'un singleton.

Il suffit à présent de prouver (ii). Pour ce faire, on raisonne comme dans [35, Thm. 2.11] . Comme le morphisme $\pi_{\bar{r}}$ est lisse, son image $U_{\text {tri }}^{\square}(\bar{r})^{\text {reg }}$ dans $X_{\text {tri }}^{\square}(\bar{r})$ est un ouvert adique par [36, Prop. 1.7.8]. L'existence de $s: U^{\square} \rightarrow \mathcal{S}^{\square}(\bar{r})$ telle que $\pi_{\bar{r}} \circ s$ égale $\left.f\right|_{U}$ composée avec $U^{\square} \rightarrow U$ montre que $U_{\mathrm{tri}}^{\square}(\bar{r})^{\mathrm{reg}}$ contient $f(U)$. Par ailleurs, $f$ étant surjective, l'inclusion $f^{-1}\left(U_{\text {tri }}^{\square}(\bar{r})^{\text {reg }}\right) \subset U$ montre que $f(U)=U_{\text {tri }}^{\square}(\bar{r})^{\text {reg }}$. Comme $f$ est un morphisme projectif, l'ensemble $f(U)=U_{\text {tri }}^{\square}(\bar{r})^{\text {reg }}$ est une partie constructible au sens de Zariski de $X_{\text {tri }}^{\square}(\bar{r})$ d'après [35, Lem. 2.14]. On déduit alors de [35, Lem. 2.13] que $U_{\text {tri }}^{\square}(\bar{r})^{\text {reg }}$ est un ouvert de Zariski de $X_{\text {tri }}^{\square}(\bar{r})$, en particulier admissible.

Le diagramme (2.1), la lissité des applications $\pi_{\bar{r}}$ et $\kappa$, ainsi que le Lemme 5.8 montrent alors que $U_{\text {tri }}^{\square}(\bar{r})^{\text {reg }}$ est lisse et que l'application $\omega^{\prime}$ est lisse, il en est donc de même de $\omega$.

Remarque 2.7. Des résultats analogues ont également été obtenus par Bergdall ([5]) et Liu ([48]).

2.3. Espaces de déformations cristallines et poids de Sen. Soit $\mathbf{k}$ un poids algébrique dominant. On note $R_{\bar{r}}^{\square, \mathbf{k}-\mathrm{cr}}=R_{\bar{r}}^{\square} / \cap \mathfrak{p}_{x}$ où l'intersection est prise sur l'ensemble des idéaux premiers $\mathfrak{p}_{x}=\operatorname{Ker}\left(x: R_{\bar{r}}^{\square} \rightarrow \overline{\mathbb{Q}}_{p}\right)$ tels que la représentation $r_{x}$ de $\mathcal{G}_{K}$ associée à $x$ est cristalline de poids de Hodge-Tate $\mathbf{k}$. C'est une $\mathcal{O}_{L}$-algèbre locale, plate, complète, noethérienne, réduite de même corps résiduel que $R_{\bar{r}}^{\square}$ et par [42], pour $A$ une $\overline{\mathbb{Q}}_{p}$-algèbre locale artinienne, un morphisme $x: R_{\bar{r}}^{\square} \rightarrow A$ se factorise à travers $R_{\bar{r}}^{\square, \mathbf{k}-\mathrm{cr}}$ si et seulement 
si $r_{x}$ est cristalline de poids de Hodge-Tate $\mathbf{k}$. On note $\mathfrak{X}_{\bar{r}}^{\square, \mathbf{k}-\mathrm{cr}}$ l'espace $\operatorname{Spf}\left(R_{\bar{r}}^{\square, \mathbf{k}-\mathrm{cr}}\right)^{\text {rig }}$ et on rappelle que ses composantes irréductibles sont en bijection canonique avec les composantes irréductibles du schéma $\operatorname{Spec}\left(R_{\bar{r}}^{\square, \mathbf{k}-\mathrm{cr}}\left[\frac{1}{p}\right]\right)$. Par [38, Lem. 7.1.9], on note que ses points sont aussi les mêmes que les points fermés de $\operatorname{Spec}\left(R_{\bar{r}}^{\square, \mathbf{k}-\mathrm{cr}}\left[\frac{1}{p}\right]\right)$.

Si $r$ est une représentation cristalline du groupe $\mathcal{G}_{K}$ sur un $L$-espace vectoriel, on appelle Frobenius cristallin linéarisé de $r$ l'endomorphisme $L$-linéaire $\varphi^{\left[K_{0}: \mathbb{Q}_{p}\right]}$ de

$$
D_{\text {cris }}(r) \otimes_{K_{0} \otimes_{\mathbb{Q}_{p}} L, \tau} L=\left(B_{\text {cris }} \otimes_{\mathbb{Q}_{p}} r\right)^{\mathcal{G}_{K}} \otimes_{K_{0} \otimes_{\mathbb{Q} p} L, \tau} L
$$

pour un plongement $\tau$ de $K_{0}$ dans $L$ (la classe de conjugaison de l'endomorphisme obtenu est alors indépendante du plongement).

Supposons désormais que $\mathbf{k}$ est strictement dominant. Soit $r$ une représentation de $\mathcal{G}_{K}$ de dimension $n$ sur $L$, cristalline de poids de Hodge-Tate k. Supposons que les valeurs propres du Frobenius cristallin linéarisé de $r$ soient deux-à-deux distinctes (en particulier $\varphi^{\left[K_{0}: \mathbb{Q}_{p}\right]}$ est diagonalisable quitte à agrandir $\left.L\right)$. Rappelons que l'on appelle raffinement de $r$ un ordre de ces valeurs propres $\varphi_{1}, \ldots, \varphi_{n}$. Le même raisonnement que dans $[4, \S 2.4 .2]$, mais en utilisant [39, Cons. 6.2.4], montre qu'il y a une bijection entre raffinements de $r$ et (systèmes de) paramètres de $r$. Soit $\varphi_{1}, \ldots, \varphi_{n}$ un raffinement de $r$, on peut alors définir une filtration croissante de $D_{\text {cris }}(r)$ par des sous- $K_{0} \otimes_{\mathbb{Q}_{p}} L$-modules libres, en posant

$$
\mathcal{F}_{i}\left(D_{\text {cris }}(r)\right)=\bigoplus_{j \leq i} D_{\text {cris }}(r)^{\varphi^{\left[K_{0}: \mathbb{Q}_{p}\right]}=\varphi_{j}} .
$$

Une telle filtration définit, comme dans $[4, \S 2.4 .1]$, un ordre sur les poids de Hodge-Tate du module filtré $D_{\mathrm{dR}}(r)_{K_{0} \otimes_{\mathbb{Q}_{p}} L, \tau} L$ pour tout $\tau \in \operatorname{Hom}(K, L)$. Le raffinement est alors dit non critique si, pour tout $\tau$, les poids de Hodge-Tate de $D_{\mathrm{dR}}(r)_{K_{0} \otimes_{\mathbb{Q}_{p}} L, \tau} L$ sont ordonnés dans l'ordre décroissant (attention que, contrairement à [4], nous supposons que le poids de $\varepsilon$ est 1$)$.

Définition 2.8. Un point $x$ de l'espace $\mathfrak{X}_{\bar{r}}^{\square, \mathbf{k}-\mathrm{cr}}$ est dit générique ${ }^{2}$ si les valeurs propres du Frobenius cristallin linéarisé de la représentation $r_{x}$ sont deux-à-deux distinctes, et si tous les raffinements de $r_{x}$ sont non critiques.

Un caractère continu $\delta \in \mathcal{T}(L)$ de $K^{\times}$dans $L^{\times}$est en particulier une fonction localement $\mathbb{Q}_{p}$-analytique sur $K^{\times}$. Sa différentielle $\mathrm{d} \delta$ en $1 \in K^{\times}$est alors une application $\mathbb{Q}_{p}$-linéaire de l'espace tangent de $K^{\times}$en 1 dans $L$. Une $\mathbb{Q}_{p}$-base de cet espace tangent est donnée par les différentielles en 1 des différents plongements $\tau: K \hookrightarrow L$. On appelle alors poids du caractère $\delta$ les coordonnées de $\mathrm{d} \delta$ dans cette base, on note ces poids $\omega_{\tau}(\delta)$. Plus précisément on a $\mathrm{d} \delta=\sum_{\tau} \omega_{\tau}(\delta) \mathrm{d} \tau$. D'après le Lemme 2.1 de [54], l'application $\left(\omega_{\tau}\right)$ ainsi construire de $\mathcal{T}(L)$ vers $L^{\left[K: \mathbb{Q}_{p}\right]}$ provient d'un morphisme d'espaces analytiques rigides $\mathcal{T}_{L} \rightarrow \mathbb{A}_{L}^{\left[K: \mathbb{Q}_{p}\right]}$

Si $V$ est une représentation continue de $\mathcal{G}_{K}$ sur un $L$-espace vectoriel de dimension finie. Si $\tau$ est un plongement de $K$ dans $L$, on appelle $\tau$-poids de Sen, les opposés des valeurs propres de l'endomorphisme $\Theta_{V}$ sur $\mathbf{D}_{\text {Sen }}(V) \otimes_{K \otimes_{\mathbb{Q}_{p}} L, \tau} L$ (cf. par exemple [6, §5.3]).

Proposition 2.9. Si $x=\left(r_{x}, \delta_{x}\right) \in X_{\text {tri }}^{\square}(\bar{r})$. Alors pour tout plongement $\tau$ de $K$ dans $L$, l'ensemble $\left\{\omega_{\tau}\left(\delta_{i, x}\right) \mid 1 \leq i \leq n\right\}$ est l'ensemble des $\tau$-poids de Sen de $r_{x}$.

2. Cette définition est une version faible de la définition de [18, §3.18], différente de la notion de généricité de [11, Déf. 5.2]. 
Démonstration. Comme $U_{\text {tri }}^{\square}(\bar{r})^{\text {reg }}$ est un ouvert de Zariski dense dans $X_{\text {tri }}^{\square}(\bar{r})$, il suffit de prouver l'assertion pour un point $x \in U_{\text {tri }}^{\square}(\bar{r})^{\text {reg }}$, puisque les applications $\omega_{\tau}$ sont analytiques d'après $[7, \S 7.2]$. Nous pouvons donc, en utilisant le diagramme (2.1), nous ramener à prouver le résultat pour les points de l'espace $\mathcal{S}^{\square}(\bar{r})$ et raisonner par récurrence pour se ramener au cas où $n=1$. Considérons donc la famille universelle $\mathcal{R}(\delta)$ sur $\mathcal{T}_{L}$. Par analyticité et Zariski-densité des caractère localement algébriques, il suffit de prouver l'assertion en un point $\delta_{x} \in \mathcal{T}$ tel que $\left.\delta_{x}\right|_{\mathcal{O}_{K}^{\times}}$est un caractère algébrique. Nous sommes donc ramenés à prouver l'assertion pour $D=\mathcal{R}\left(\delta_{x}\right)$ avec $\delta_{x}(z)=\prod_{\tau} \tau(x)^{k_{\tau}}$ lorsque $z \in \mathcal{O}_{K}^{\times}$. Un calcul facile montre que $\omega_{\tau}\left(\delta_{x}\right)=k_{\tau}$. Il reste donc à prouver que $\Theta_{D}$ agit sur $\mathbf{D}_{\text {Sen }}\left(\mathcal{R}\left(\delta_{x}\right)\right)$ par multiplication par $k_{\tau}$. Quitte à multiplier $\delta_{x}$ par un caractère non ramifié, on peut supposer que $\delta_{x}$ est à valeurs dans $\mathcal{O}_{k(x)}^{\times}$, et donc que $D$ est le $\left(\varphi, \Gamma_{K}\right)$ module associé au caractère $\delta_{x} \circ \mathrm{rec}^{-1}$. Or on sait qu'un tel caractère a pour $\tau$-poids de Hodge-Tate $k_{\tau}$.

Lemme 2.10. Soit $X$ un espace rigide analytique réduit et $D$ un $\left(\varphi, \Gamma_{K}\right)$-module sur $X$. Soit $D_{1}$ un sous- $\mathcal{R}_{X}$-module de $D$ stable par $\varphi$ et $\Gamma_{K}$. Supposons qu'il existe un morphisme injectif de $(\varphi, \Gamma)$-modules

$$
\alpha: D_{1} \longrightarrow G_{1}
$$

où $G_{1}$ est un $\left(\varphi, \Gamma_{K}\right)$-module localement sur $X$ libre de rang 1 . Si $i_{x}^{*}(\alpha)$ est un isomorphisme pour $x$ dans une partie Zariski-dense de $X$, alors le morphisme $\alpha$ est un isomorphisme.

Démonstration. C'est exactement le contenu de la preuve de [39, Cor. 6.3.10].

Lemme 2.11. Soit $x=\left(r_{x}, \delta_{x}\right)$ un point de $X_{\mathrm{tri}}^{\square}(\bar{r})$ tel que $r_{x}$ est une représentation cristalline générique. Alors le caractère $\delta_{x}$ est localement algébrique, strictement dominant et $x \in U_{\mathrm{tri}}^{\square}(\bar{r})^{\mathrm{reg}}$.

Démonstration. La preuve qui suit est dans le même esprit que [11, Prop. 9.2]. Comme dans la preuve du Théorème 2.6, il existe d'après [39, Cor. 6.3.10] un morphisme propre et birationnel $\tilde{X}=\tilde{X}_{\text {tri }}^{\square}(\bar{r}) \rightarrow X_{\text {tri }}^{\square}(\bar{r})$, ainsi qu'une filtration $F_{\bullet}$ de $\tilde{D}_{X}$, l'image inverse de $D_{X}=\mathbf{D}_{\text {rig }}^{\dagger}\left(r_{X}\right)$ par des sous- $\mathcal{R}_{X}$-modules stables par $\varphi$ et $\Gamma_{K}$ telle que $F_{0}=0$ et $F_{n}=\tilde{D}_{X}$, une famille $\left(\mathcal{L}_{i}\right)_{1 \leq i \leq n}$ de $\mathcal{O}_{\tilde{X}}$-modules inversibles ainsi que des morphismes injectifs

$$
\alpha_{i}: F_{i} / F_{i-1} \longrightarrow \mathcal{R}_{\tilde{X}}\left(\delta_{i, \tilde{X}}\right) \otimes_{\mathcal{O}_{\tilde{X}}} \mathcal{L}_{i}
$$

qui sont des isomorphismes sur une partie Zariski-dense de $\tilde{X}$. Par [39, Cor. 6.3.10.(2')], $\alpha_{1}$ est alors automatiquement un isomorphisme. Fixons $\tilde{x}$ un antécédent de $x$ dans $\tilde{X}$. En spécialisant le $\left(\varphi, \Gamma_{K}\right)$-module $D_{\tilde{X}}$ en $\tilde{x}$, on obtient en particulier une injection

$$
\alpha_{1, x}: \mathcal{R}_{k(x), K}\left(\delta_{1, x}\right) \hookrightarrow i_{\tilde{x}}^{*}\left(D_{\tilde{X}}\right) \simeq i_{x}^{*}\left(D_{X}\right)
$$

de $\left(\varphi, \Gamma_{K}\right)$-modules sur $k(x)$. Considérons le saturé de l'image de $\alpha_{1, x}$, c'est-à-dire l'intersection $\operatorname{Im}\left(\alpha_{1, x}\right)\left[\frac{1}{t}\right] \cap i_{x}^{*}\left(D_{x}\right)$. D'après [4, Prop. 2.2.2] et [22], il s'agit d'un $\left(\varphi, \Gamma_{K}\right)$-module de rang 1 , donc de la forme $\mathcal{R}_{k(x), K}\left(\delta_{1}^{\prime}\right)$ avec $\delta_{1}^{\prime} \in \mathcal{T}(k(x))$. Comme $\mathcal{R}_{k(x), K}\left(\delta_{1, x}\right)$ est isomorphe à un sous- $\left(\varphi, \Gamma_{K}\right)$-module de $\mathcal{R}_{k(x), K}\left(\delta_{1}^{\prime}\right)$, il existe $\mathbf{n} \in \mathbb{N}^{\left[K: \mathbb{Q}_{p}\right]}$ tel que $\delta_{1, x}=x^{\mathbf{n}} \delta_{1}^{\prime}$. Comme $i_{x}^{*}\left(D_{X}\right)$ est le $(\varphi, \Gamma)$-module de la représentation cristalline $r_{x}$, $\delta_{1}^{\prime}$ est le premier terme d'un paramètre $\delta^{\prime}=\left(\delta_{1}^{\prime}, \ldots, \delta_{n}^{\prime}\right)$ de $i_{x}^{*}\left(D_{X}\right)$. Les caractères $\delta_{x}$ et $\delta^{\prime}$ sont localement algébriques de poids respectifs $\mathbf{k}=\left(k_{\tau, i}\right)$ et $\mathbf{k}^{\prime}=\left(k_{\tau, i}^{\prime}\right)$ et comme la représentation galoisienne associée au $(\varphi, \Gamma)$-module $i_{x}^{*}\left(D_{X}\right)$ est générique, $\delta^{\prime}$ est un caractère dominant. Par ailleurs, on déduit de la Proposition 2.9 que les ensembles $\left\{k_{\tau, i} \mid 1 \leq i \leq n\right\}$ 
et $\left\{k_{\tau, i}^{\prime} \mid 1 \leq i \leq n\right\}$ sont égaux pour tout plongement $\tau$ de $K$ dans $L$, en particulier l'ensemble $\left\{k_{\tau, i}^{\prime} \mid 1 \leq i \leq n\right\} \cup\left\{k_{\tau, 1}\right\}$ est de cardinal $n$. Dans la suite d'inégalités

$$
k_{\tau, 1} \geq k_{\tau, 1}^{\prime}>k_{\tau, 2}^{\prime}>\cdots>k_{\tau, n}^{\prime}
$$

la première est donc nécessairement une égalité. Ceci étant vrai pour tout $\tau$, on a $\delta_{1, x}=$ $\delta_{1, x}^{\prime}$, donc $\mathcal{R}_{k(x), K}\left(\delta_{1, x}\right)$ est un sous-module saturé de $i_{x}^{*}\left(D_{X}\right)$. Par [4, Lem. 2.2.3], on en conclut que le $\mathcal{R}_{k(x), K}$-module $i_{x}^{*}\left(D_{X}\right) / \mathcal{R}_{k(x), K}\left(\delta_{1, x}\right)$ est libre. Ceci implique que, sur un voisinage affinoïde $U_{1}$ de $\tilde{x}$ dans $\tilde{X}_{\text {tri }}^{\square}(\bar{r})$, le quotient $\operatorname{Coker}\left(\alpha_{1}\right)$ est un $\left(\varphi, \Gamma_{K}\right)$-module. En particulier, le lemme 2.10 montre que l'application $\alpha_{2}$ est un isomorphisme sur $U_{1}$. Par récurrence, on construit une suite décroissante de voisinages $\left(U_{i}\right)_{1 \leq i \leq n}$ de $\tilde{x}$ telle que, sur $U_{i}$, les application $\alpha_{j}$ pour $j \leq i$ soient des isomorphismes. On conclut au final que $\delta_{x}$ est un paramètre du $\left(\varphi, \Gamma_{K}\right)$-module $i_{x}^{*}\left(D_{X}\right)$, c'est-à-dire que le point $x$ appartient à $U_{\text {tri }}^{\square}(\bar{r})^{\text {reg. }}$.

Rappelons que $T \cong \mathbb{G}_{m}^{n}$ est le tore diagonal de $\mathrm{GL}_{n}$. On introduit un automorphisme $\iota_{K}$ de $\widehat{T(K)}$ en posant

$$
\iota_{K}\left(\delta_{1}, \cdots, \delta_{n}\right)=\delta_{B} \cdot\left(\delta_{1}, \cdots, \delta_{i} \cdot\left(\varepsilon \circ \operatorname{rec}_{K}\right)^{i-1}, \cdots, \delta_{n} \cdot\left(\varepsilon \circ \operatorname{rec}_{K}\right)^{n-1}\right)
$$

où $\delta_{B}=\left.|\cdot|_{K}^{n-1} \otimes|\cdot|_{K}^{n-3} \otimes \cdots \otimes|\cdot|\right|_{K} ^{1-n}$ est le caractère module de $B(K)$. La raison d'être de cette paramétrisation est la suivante. Normalisons la correspondance locale de Langlands pour $\mathrm{GL}_{n}(K)$ de telle sorte que la représentation de Weil $\chi_{1} \oplus \cdots \oplus \chi_{n}$ corresponde à l'induite parabolique lisse non normalisée $\operatorname{Ind}_{B(K)}^{\mathrm{GL}_{n}(K)}\left(\delta_{B}^{1 / 2} \cdot\left(\chi_{1} \circ \operatorname{rec}_{K} \cdots \otimes \chi_{n} \circ \operatorname{rec}_{K}\right)\right)$. Si $r$ est une représentation cristalline de dimension $n$ de $\mathcal{G}_{K}$ sur $L$ de poids de HodgeTate $\mathbf{k}=\left(k_{\tau, i}\right)_{1 \leq i \leq n, \tau: K \hookrightarrow L}$ strictement dominant, et $\pi$ une représentation non ramifiée irréductible de $\mathrm{GL}_{n}(K)$ sur $\overline{\mathbb{Q}}_{p}$ telle que la représentation de Weil associée à $r([30])$ corresponde à $\pi|\operatorname{det}|_{\frac{1-n}{2}}^{\frac{1}{2}}$ par la correspondance de Langlands normalisée comme ci-dessus, alors $\left(\delta_{1}, \cdots, \delta_{n}\right)$ est un paramètre de $r$ si et seulement si $\iota_{K}\left(\delta_{1}, \cdots, \delta_{n}\right)$ est un système de valeurs propres de $T(K)$ agissant sur $J_{B}\left(\pi \otimes_{\overline{\mathbb{Q}}_{p}} W_{\mathbf{n}}\right)$ où $W_{\mathbf{n}}$ est la représentation algébrique irréductible de $\operatorname{Res}_{K / \mathbb{Q}_{p}}\left(\mathrm{GL}_{n}\right)$ sur $\overline{\mathbb{Q}}_{p}$ de plus haut poids $\mathbf{n}=\left(k_{\tau, i}+i-1\right)_{1 \leq i \leq n, \tau: K \hookrightarrow \overline{\mathbb{Q}}_{p}}$ relativement à $B$ et $J_{B}$ est le foncteur de Jacquet comme étendu par Emerton aux représentations localement algébriques, cf. [26, Prop. 4.3.6].

2.4. Espaces de formes automorphes. Fixons $F$ un corps CM, i.e. une extension quadratique totalement imaginaire d'un corps $F^{+} \subset F$ totalement réel et notons $c$ l'unique automorphisme non trivial de $F$ fixant $F^{+}$. Nous supposons de plus que toutes les places de $F^{+}$divisant $p$ sont complètement décomposées dans $F$ et que l'extension $F / F^{+}$est non ramifiée en toute place finie. Pour toute place finie $w$ de $F$, on fixe une uniformisante $\varpi_{w}$ de $F_{w}$. On choisit désormais le corps $L$ tel que pour toute place $v$ de $F^{+}$divisant $p$, on a $\left|\operatorname{Hom}\left(F_{v}^{+}, L\right)\right|=\left[F_{v}^{+}: \mathbb{Q}_{p}\right]$.

Supposons désormais $n$ impair ou $\left[F^{+}: \mathbb{Q}\right]$ pair. Soit $G$ un groupe unitaire à $n$ variables défini sur $F^{+}$, déployé par $F$, quasi-déployé en toute place finie et tel que $G\left(F^{+} \otimes_{\mathbb{Q}} \mathbb{R}\right)$ est compact. On fixe $U^{p} \subset G\left(\mathbb{A}_{F^{+}}^{p \infty}\right)$ un sous-groupe compact ouvert de la forme $\prod_{v} U_{v}$ où $U_{v}$ est un sous-groupe compact ouvert de $G\left(F_{v}\right)$. Ici $\mathbb{A}_{F^{+}}^{p \infty}$ désigne les adèles finis de $F^{+}$hors les places divisant $p$. On fixe aussi un ensemble fini $S$ de places finies de $F^{+}$contenant l'ensemble $S_{p}$ des places divisant $p$ ainsi que l'ensemble des places $v$ telles que $U_{v}$ n'est pas hyperspécial, et on suppose que si $v \in S$, alors $v$ est totalement décomposée dans $F$ (de manière équivalente, on suppose $U_{v}$ hyperspécial en toutes les places de $F^{+}$inertes dans $F)$. 
Si $v \in S$, fixons $\tilde{v}$ une place de $F$ divisant $v$ et notons $\tilde{v}^{c}$ son image par l'automorphisme c. L'inclusion de $F^{+}$dans $F$ induit donc deux isomorphismes $F_{v}^{+} \stackrel{\sim}{\rightarrow} F_{\tilde{v}}$ et $F_{v}^{+} \stackrel{\sim}{\rightarrow} F_{\tilde{v}^{c}}$. Fixons de plus un isomorphisme de groupes algébriques $G \times_{F^{+}} F \simeq \mathrm{GL}_{n, F}$. Si $v$ est décomposée dans $F$ et $w$ est une place de $F$ divisant $v$, on obtient un isomorphisme $i_{w}: G\left(F_{v}^{+}\right) \stackrel{\sim}{\rightarrow} \operatorname{GL}_{n}\left(F_{w}\right)$. On suppose de plus que, pour $v \notin S$, on a $U_{v}=i_{w}^{-1}\left(\mathrm{GL}_{n}\left(\mathcal{O}_{F_{w}}\right)\right)$, condition qui ne dépend pas du choix de $w$ divisant $v$. Pour $v \in S$, notons $K_{v}$ le sousgroupe compact maximal de $G\left(F_{v}^{+}\right)$image réciproque par $i_{\tilde{v}}$ du groupe $\operatorname{GL}_{n}\left(\mathcal{O}_{F_{\tilde{v}}}\right)$. On note aussi $G_{p}=\prod_{v \in S_{p}} G\left(F_{v}^{+}\right)$et $K_{p}=\prod_{v \in S_{p}} K_{v}$. On désigne par $B_{v}$ l'image inverse par $i_{\tilde{v}}$ du sous-groupe des matrices triangulaires supérieures de $\operatorname{GL}_{n}\left(F_{\tilde{v}}\right)$ et $T_{v}$ et $N_{v}$ les images inverses des sous-groupes de matrices diagonales et unipotentes supérieures. On pose alors

$$
\begin{array}{ll}
B_{p}=\prod_{v \in S_{p}} B_{v}, & B_{p}^{0}=B_{p} \cap K_{p}, \\
N_{p}=\prod_{v \in S_{p}} N_{v}, & N_{p}^{0}=N_{p} \cap K_{p}, \\
T_{p}=\prod_{v \in S_{p}} T_{v}, & T_{p}^{0}=T_{p} \cap K_{p} .
\end{array}
$$

Enfin, on note $\bar{B}_{p}$ et $\bar{N}_{p}$ les sous-groupes de Borel et unipotent opposés à $B_{p}$ et $N_{p}$. Si $I \subset\{1, \cdots, n\}$ et $v \in S_{p}$, on note $P_{v, I}, \bar{P}_{v, I}$ et $L_{v, I}$ les images réciproques de $P_{I}\left(F_{\tilde{v}}\right)$, $\bar{P}_{I}\left(F_{\tilde{v}}\right)$ et $L_{I}\left(F_{\tilde{v}}\right)$.

Soit $\lambda=\left(\lambda_{\tau, i}\right) \in\left(\mathbb{Z}^{n}\right)^{\operatorname{Hom}\left(F^{+}, L\right)}$. Un élément $\delta \in \widehat{T}_{p}(L)$ est dit algébrique de poids $\lambda$ s'il est de la forme $\bigotimes_{v \in S_{p}} \delta_{\lambda_{v}}$ où $\lambda_{v}=\left(\lambda_{\tau, i}\right)_{1<i<n, \tau: F_{v}^{+} \hookrightarrow L}$. Il est dit localement algébrique de poids $\lambda$ s'il coïncide avec $\bigotimes_{v \in S_{p}} \delta_{\lambda_{v}}$ sur un sous-groupe ouvert de $T_{p}$.

Si $V=\prod_{v} V_{v}$ est un sous-groupe compact ouvert de $G\left(\mathbb{A}_{F^{+}}^{\infty}\right)$ et $\sigma$ une représentation continue de $\prod_{v} V_{v}$ sur un $\mathcal{O}_{L^{-}}$-module topologique, on note $S(V, \sigma)$ le $\mathcal{O}_{L^{-} \text {-module des }}$ fonctions continues $f: G\left(F^{+}\right) \backslash G\left(\mathbb{A}_{F^{+}}^{\infty}\right) \rightarrow \sigma$ telles que $f(g \cdot u)=u^{-1}(f(g))$ pour tout $g \in \mathbb{A}_{F^{+}}^{\infty}$ et $u \in V$.

Si $v$ est une place de $F^{+}$n'appartenant pas à $S$ et décomposée dans $F$, on note $\mathbb{T}_{v}$ l'algèbre de Hecke sphérique du groupe $G\left(F_{v}^{+}\right)$relativement à $U_{v}$, c'est-à-dire

$$
\mathbb{T}_{v}=\mathcal{O}_{L}\left[U_{v} \backslash G\left(F_{v}^{+}\right) / U_{v}\right]
$$

On pose alors $\mathbb{T}^{S}=\lim _{\longrightarrow}\left(\bigotimes_{v \in I} \mathbb{T}_{v}\right)$, la limite inductive étant prise sur l'ensemble des parties finies de places finies de $F^{+}$décomposées dans $F$ et d'intersection nulle avec $S$. L'algèbre $\mathbb{T}^{S}$ agit alors naturellement sur $S\left(U^{p} U_{p}, \sigma\right)$ pour tout sous-groupe compact ouvert $U_{p}$ de $G_{p}$. De plus cette action est fonctorielle en $U_{p}$ et en $\sigma$ (en un sens évident). Si $w$ est une place de $F$ divisant $v$, on note $T_{w, i}$ l'élément de $\mathbb{T}_{v}$ de support $U_{v} \gamma_{w, i} U_{v}$ où $\gamma_{w, i}$ est l'élément de $G\left(F_{v}^{+}\right)$image réciproque par $i_{w}$ de la matrice diagonale

$$
\beta_{i}\left(\varpi_{w}\right)=(\underbrace{\varpi_{w}, \cdots, \varpi_{w}}_{i}, 1, \cdots, 1) .
$$

Considérons un corps quelconque $C$ et $\theta: \mathbb{T}^{S} \rightarrow C$ un morphisme d'anneaux. Si $\rho$ est une représentation $C$-linéaire de $\mathcal{G}_{F}=\operatorname{Gal}(\bar{F} / F)$ sur un $C$-espace vectoriel de dimension finie, on dit que $\rho$ est associée à $\theta$ si pour toute place $w$ de $F$ au-dessus d'une place de $F^{+}$ n'appartenant pas à $S$ et complètement décomposée dans $F$, le polynôme caractéristique de $\rho\left(\right.$ Frob $\left._{w}\right)$ est donné par

$$
X^{n}+\cdots+(-1)^{j}(N w)^{j(j-1) / 2} T_{w, j} X^{n-j}+\cdots+(-1)^{n}(N w)^{n(n-1) / 2} T_{w, n}
$$


où Frob ${ }_{w}$ est un Frobenius géométrique en $w$ et $N w$ le cardinal du corps résiduel de $F_{w}$. Si $\pi$ est une représentation automorphe de $G\left(\mathbb{A}_{F^{+}}\right)$non ramifiée hors de $S$, il existe une unique $\overline{\mathbb{Q}}_{p}$-représentation semi-simple $\rho_{\pi}$ de $G_{F}$ associée au caractère de $\mathbb{T}^{S}$ donné par l'action de $\mathbb{T}^{S}$ sur la $\overline{\mathbb{Q}}_{p}$-droite $\left(\pi^{S}\right)^{U^{S}} \otimes_{\mathbb{C}} \overline{\mathbb{Q}}_{p}$ où $U^{S}=\prod_{v \notin S} U_{v}$ (voir [20, Thm. 3.2.3]).

On dit qu'un idéal maximal $\mathfrak{m}$ de l'algèbre $\mathbb{T}^{S}$ est automorphe de niveau modéré $U^{p}$ s'il existe une représentation algébrique $\sigma$ de $K_{p}$ sur un $\mathcal{O}_{L}$-module (avec topologie discrète) qui est irréductible après extension des scalaires de $\mathcal{O}_{L}$ à $\overline{\mathbb{Q}}_{p}$ (donc de dimension finie sur $\left.\overline{\mathbb{Q}}_{p}\right)$ et un sous-groupe compact ouvert $U_{p}$ de $G_{p}$ tels que $S\left(U^{p} U_{p}, \sigma\right)_{\mathfrak{m}} \neq 0$. En particulier par ce qui précède (en procédant par exemple comme dans $[27, \S 3]$ ou $[4$, $\S 6.2 .3]$ ) il existe alors aussi une représentation continue $\bar{\rho}_{\mathfrak{m}}$ de $G_{F}$ sur un $\mathbb{T}^{S} / \mathfrak{m}$-espace vectoriel de dimension $n$ associée au caractère $\mathbb{T}^{S} \rightarrow \mathbb{T}^{S} / \mathfrak{m}$. On dit que l'idéal maximal $\mathfrak{m}$ est de plus non Eisenstein si $\bar{\rho}_{\mathfrak{m}}$ est absolument irréductible. Fixons désormais un tel idéal maximal $\mathfrak{m}$ automorphe de niveau modéré $U^{p}$ non Eisenstein et une représentation algébrique $\sigma$ comme ci-dessus. On vérifie facilement à partir de (2.3) et des définitions que les représentations irréductibles $\bar{\rho}_{\mathfrak{m}}^{\vee} \circ c$ et $\bar{\rho}_{\mathfrak{m}} \otimes \bar{\varepsilon}^{n-1}$ sont isomorphes (où $\bar{\varepsilon}$ est la réduction de $\varepsilon$ modulo $p$ ). Quitte à agrandir $L$, on suppose $\mathbb{T}^{S} / \mathfrak{m}=k_{L}$. On suppose également $p>2$.

Soit $\bar{\rho}$ une représentation continue absolument irréductible de $\mathcal{G}_{F}$ sur un $k_{L}$-espace vectoriel de dimension $n$ telle que $\bar{\rho}^{\vee} \circ c \cong \bar{\rho} \otimes \bar{\varepsilon}^{n-1}$ et $\bar{\rho}$ est non ramifiée hors de $S$. Alors le foncteur associant à toute $\mathcal{O}_{L}$-algèbre locale artinienne $A$ de corps résiduel $k_{L}$ l'ensemble des classes d'isomorphisme de déformations $\rho_{A}$ de $\bar{\rho}$ à $A$ non ramifiées en dehors de $S$ et vérifiant $\rho_{A}^{\vee} \circ c \simeq \rho_{A} \otimes \varepsilon^{n-1}$ est pro-représentable par une $\mathcal{O}_{L}$-algèbre locale complète noethérienne de corps résiduel $k_{L}$ notée $R_{\bar{\rho}, S}$.

Pour tout sous-groupe compact ouvert $U_{p}$ de $G_{p}$ on note $\mathbb{T}^{S}\left(U^{p} U_{p}, \sigma\right)_{\mathfrak{m}}$ l'image de $\mathbb{T}^{S}$ dans l'algèbre des endomorphismes de $S\left(U^{p} U_{p}, \sigma\right)_{\mathfrak{m}}$. De [60, Prop. 6.7] (notons que l'on utilise ici $p \neq 2$ ) on déduit qu'il existe un morphisme canonique de $\mathcal{O}_{L}$-algèbres locales noethériennes complètes

$$
\psi: R_{\bar{\rho}_{\mathfrak{m}}, S} \longrightarrow \mathbb{T}^{S}\left(U^{p} U_{p}, \sigma\right)_{\mathfrak{m}}
$$

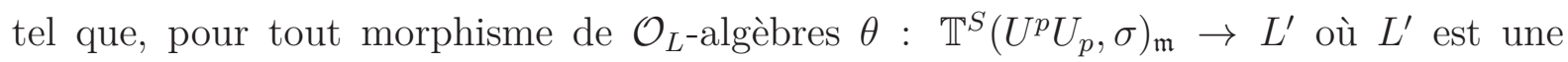
extension finie de $L$, la composée $\theta \circ \psi$ définit une déformation de $\bar{\rho}_{\mathfrak{m}}$ associée au caractère $\theta$.

On définit l'espace de cohomologie complétée du groupe $G$ de niveau modéré $U^{p}$, ou espace des formes automorphes $p$-adiques de niveau modéré $U^{p}$, comme la complétion de l'espace $\lim _{U_{p}} S\left(U^{p} U_{p}, L\right)$ pour la norme définie par le réseau $\lim _{U_{p}} S\left(U^{p} U_{p}, \mathcal{O}_{L}\right)$, la limite inductive étant prise sur les sous-groupes compacts ouverts $U_{p}$ de $G_{p}$. On note $\widehat{S}\left(U^{p}, L\right)$ cet espace de Banach $p$-adique, qui s'identifie également à l'espace des fonctions continues $G\left(F^{+}\right) \backslash G\left(\mathbb{A}_{F^{+}}^{\infty}\right) / U^{p} \rightarrow L$ muni de la norme sup. Il est naturellement muni d'une action continue de $G_{p}$ par translation à droite sur ces fonctions et d'une action continue de $\mathbb{T}^{S}$ qui commute à celle de $G_{p}$, ces actions préservant la boule unité $\widehat{S}\left(U^{p}, \mathcal{O}_{L}\right)$ (i.e. l'adhérence de $\lim _{U_{p}} S\left(U^{p} U_{p}, \mathcal{O}_{L}\right)$ dans $\left.\widehat{S}\left(U^{p}, L\right)\right)$.

Notons que ces actions s'étendent au localisé $\widehat{S}\left(U^{p}, L\right)_{\mathfrak{m}}$, un espace de Banach $p$-adique sur $L$ qui s'identifie au complété de $\lim _{U_{p}} S\left(U^{p} U_{p}, L\right)_{\mathfrak{m}}$ par rapport à $\varliminf_{\longrightarrow} S\left(U^{p} U_{p}, \mathcal{O}_{L}\right)_{\mathfrak{m}}$, dont elles préservent la boule unité $\widehat{S}\left(U^{p}, \mathcal{O}_{L}\right)_{\mathfrak{m}}$. On en déduit que $\widehat{S}\left(U^{p}, L\right)_{\mathfrak{m}}$ et $\widehat{S}\left(U^{p}, \mathcal{O}_{L}\right)_{\mathfrak{m}}$ sont munis via $\psi$ d'une action continue de la $\mathcal{O}_{L}$-algèbre $R_{\bar{\rho}_{\mathrm{m}}, S}$ commutant à l'action de $G_{p}$ (et qui coïncide sur $\mathcal{O}_{L}$ avec la multiplication scalaire). 
Si $v$ est une place de $F^{+}$divisant $p$, on note $\bar{\rho}_{\tilde{v}}$ la restriction de $\bar{\rho}_{\mathfrak{m}}$ au groupe de décomposition en $\tilde{v}$. Rappelons que l'on a défini un sous-espace analytique rigide fermé et réduit $X_{\text {tri }}^{\square}\left(\bar{\rho}_{\tilde{v}}\right)$ de $\operatorname{Spf}\left(R_{\bar{\rho}_{\tilde{v}}}^{\square}\right)^{\text {rig }} \times \widehat{T}_{v, L}$ correspondant aux déformations triangulines de $\bar{\rho}_{\tilde{v}}$ (Définition 2.4) ainsi qu'un ouvert admissible Zariski-dense $U_{\text {tri }}^{\square}\left(\bar{\rho}_{\tilde{v}}\right)^{\text {reg }}$ de $X_{\text {tri }}^{\square}\left(\bar{\rho}_{\tilde{v}}\right)$ (Théorème 2.6). On note $X_{\text {tri }}^{\square}\left(\bar{\rho}_{p}\right)$ l'espace analytique rigide produit $\prod_{v \in S_{p}} X_{\text {tri }}^{\square}\left(\bar{\rho}_{\tilde{v}}\right)$, qui est donc un sous-espace analytique rigide fermé et réduit de $\operatorname{Spf}\left(R_{\bar{\rho}_{p}}^{\square}\right)^{\text {rig }} \times \widehat{T}_{p, L}$ où $R_{\bar{\rho}_{p}}^{\square}$ désigne $\widehat{\bigotimes}_{v \in S_{p}} R_{\bar{\rho}_{\tilde{v}}}^{\square}$. De même, on note $U_{\text {tri }}^{\square}\left(\bar{\rho}_{p}\right)^{\text {reg }}=\prod_{v \in S_{p}} U_{\text {tri }}^{\square}\left(\bar{\rho}_{\tilde{v}}\right)^{\text {reg }}$, un ouvert admissible et Zariski-dense de $X_{\text {tri }}^{\square}\left(\bar{\rho}_{p}\right)$.

\section{La variété De HeCKe-TAYlor-Wiles}

Nous conservons les notations et hypothèses de la partie précédente.

3.1. Vecteurs localement $R$-analytiques. Soit $R$ une $\mathcal{O}_{L}$-algèbre locale complète noethérienne commutative de corps résiduel $k_{L}$. On munit $R$ de sa topologie $\mathfrak{m}_{R}$-adique où $\mathfrak{m}_{R}$ est l'idéal maximal de $R$. On désigne par la lettre $G$ un groupe de Lie $p$-adique, par $G_{0}$ un sous-groupe compact ouvert de $G$, par $D\left(G_{0}, L\right)$ les distributions localement $\left(\mathbb{Q}_{p^{-}}\right)$analytiques sur $G_{0}$ à valeurs dans $L$ (i.e. le dual continu des fonctions localement $\mathbb{Q}_{p}$-analytiques $G_{0} \rightarrow L$, cf. [55], [57], [25]) et par $R \llbracket G_{0} \rrbracket$ l'algèbre d'Iwasawa de $G_{0}$ à coefficients dans $R$. Rappelons que $\mathcal{O}_{L} \llbracket G_{0} \rrbracket[1 / p]$ est le dual continu de l'espace de Banach des fonctions continues $G_{0} \rightarrow L$.

Définition 3.1. Soit $\Pi$ un espace de Banach sur $L$ muni d'une action continue de $G$ et d'une action de l'algèbre $R$ commutant à $G$ dont la restriction à $\mathcal{O}_{L}$ est la multiplication scalaire et telle que l'application canonique $R \times \Pi \rightarrow \Pi$ est continue. On dit que $\Pi$ est une représentation continue $R$-admissible si son dual continu $\Pi^{\prime}$ est un $R \llbracket G_{0} \rrbracket[1 / p]$-module de type fini.

Précisons que l'action de $G$ sur $\Pi^{\prime}$ est par translation à droite sur les fonctions et l'action de $R$ donnée par $(r \cdot f)(v)=f(r \cdot v)\left(r \in R, f \in \Pi^{\prime}, v \in \Pi\right)$.

Exemple 3.1. Les espaces de Banach $\widehat{S}\left(U^{p}, L\right)$ et $\widehat{S}\left(U^{p}, L\right)_{\mathfrak{m}}$ du $\S 2.4$ sont des représentations continues $R_{\bar{\rho}_{\mathfrak{m}}, S}$-admissibles de $G_{p}$ sur $L$ (car leurs duaux sont en fait des $\mathcal{O}_{L} \llbracket K_{p} \rrbracket[1 / p]$-modules de type fini).

Fixons dans la suite une présentation $\mathcal{O}_{L} \llbracket X_{1}, \cdots, X_{s} \rrbracket \rightarrow R$ de $R$. On a un isomorphisme de $\mathcal{O}_{L}$-algèbres topologiques $\mathcal{O}_{L} \llbracket X_{1}, \cdots, X_{s} \rrbracket \simeq \mathcal{O}_{L} \llbracket \mathbb{Z}_{p}^{s} \rrbracket$ défini par $X_{i} \mapsto \delta_{i}-1$ où $\left(\delta_{1}, \cdots, \delta_{s}\right)$ désigne la base canonique de $\mathbb{Z}_{p}^{s}$. Donc $\Pi^{\prime}$ (comme dans la Définition 3.1) est en fait un $\mathcal{O}_{L} \llbracket \mathbb{Z}_{p}^{s} \times G_{0} \rrbracket$-module de type fini. Par [52, Thm. 3.5], on voit que $\Pi$ est une représentation continue admissible de $\mathbb{Z}_{p}^{s} \times G$ sur $L$ et que la Définition 3.1 est indépendante du choix du sous-groupe ouvert compact $G_{0}$ de $G$. Inversement, toute représentation continue admissible de $\mathbb{Z}_{p}^{s} \times G$ sur $L$ dont l'action induite de $\mathcal{O}_{L} \llbracket \mathbb{Z}_{p}^{s} \rrbracket$ se factorise par $R$ est une représentation continue $R$-admissible de $G$.

Définition 3.2. Soit $\Pi$ une représentation continue $R$-admissible de $G$ sur $L$. Un élément de $\Pi$ est dit localement $R$-analytique s'il est localement analytique pour l'action de $\mathbb{Z}_{p}^{s} \times$ $G$ au sens de $[55, \S 7]$ (on montre ci-dessous que cela ne dépend pas du choix de la présentation choisie $\mathcal{O}_{L} \llbracket \mathbb{Z}_{p}^{s} \rrbracket \rightarrow R$ de $R$ ). 
On note $\Pi^{R-\text { an }}$ le sous-espace de ces vecteurs localement analytiques. Rappelons que c'est un $L$-espace vectoriel localement convexe de type compact stable sous l'action de $\mathbb{Z}_{p}^{s} \times G([55, \S 7])$.

Notons $R^{\text {rig }}=\mathcal{O}\left(\operatorname{Spf}(R)^{\text {rig }}\right)$ l'algèbre des fonctions globales sur l'espace analytique rigide $\operatorname{Spf}(R)^{\text {rig }}$. Concrètement, on peut en donner la description suivante (cf. [38, Def. 7.1.3]). Pour tout $n \geq 1$, on a dans $R[1 / p]$ le sous-anneau $R\left[\mathfrak{m}_{R}^{n} / \varpi\right]$ (avec des notations évidentes) dont on note $R_{n}^{\circ}$ la complétion $\varpi$-adique. On a alors

$$
R^{\text {rig }}={\underset{n}{\lim }}_{n}\left(R_{n}^{\circ} \otimes_{\mathcal{O}_{L}} L\right) \text {. }
$$

De plus [38, Lem. 7.2.2] montre que $R^{\text {rig }}$ est une $L$-algèbre de Fréchet-Stein au sens de $[55, \S 3]$. Enfin, si $S$ est une autre $\mathcal{O}_{L}$-algèbre locale complète noethérienne, on déduit de [38, Prop. 7.2.4.g)] un isomorphisme

$$
R^{\mathrm{rig}} \widehat{\otimes}_{L} S^{\mathrm{rig}} \simeq\left(R \widehat{\otimes}_{\mathcal{O}_{L}} S\right)^{\mathrm{rig}}
$$

(où le produit tensoriel à gauche est dans la catégorie des $L$-algèbres de Fréchet-Stein et à droite dans la catégorie des $\mathcal{O}_{L}$-algèbres locales complètes noethériennes). Rappelons par ailleurs que $\mathcal{O}_{L} \llbracket \mathbb{Z}_{p}^{s} \rrbracket^{\text {rig }}$ s'identifie à l'algèbre $D\left(\mathbb{Z}_{p}^{s}, L\right)$ des distributions localement analytiques sur $\mathbb{Z}_{p}^{s}$ à valeurs dans $L$ (voir par exemple [54, Thm. 2.2]) et que l'on a un isomorphisme de $L$-algèbres de Fréchet-Stein $D\left(\mathbb{Z}_{p}^{s} \times G_{0}, L\right) \cong D\left(\mathbb{Z}_{p}^{s}, L\right) \widehat{\otimes}_{L} D\left(G_{0}, L\right)$ ([57, Prop. A.3]) où $D\left(\mathbb{Z}_{p}^{s} \times G_{0}, L\right)$ est l'algèbre des distributions localement analytiques sur $\mathbb{Z}_{p}^{s} \times G_{0}$ à valeurs dans $L$. La surjection $\mathcal{O}_{L} \llbracket \mathbb{Z}_{p}^{s} \rrbracket \rightarrow R$ induit une application continue d'algèbres de Fréchet-Stein $D\left(\mathbb{Z}_{p}^{s}, L\right) \cong \mathcal{O}_{L} \llbracket \mathbb{Z}_{p}^{s} \rrbracket^{\text {rig }} \rightarrow R^{\text {rig }}$ (qui en fait est encore surjective par le Lemme 5.1 en appendice). Par [55, Thm. 7.1], le dual continu de l'espace des vecteurs localement analytiques de $\Pi$ pour l'action de $\mathbb{Z}_{p}^{s} \times G$ s'identifie à $D\left(\mathbb{Z}_{p}^{s} \times G_{0}, L\right) \otimes_{\mathcal{O}_{L} \llbracket \mathbb{Z}_{p}^{s} \times G_{0} \rrbracket} \Pi^{\prime}$.

Lemme 3.3. Soit $\Pi$ une représentation continue $R$-admissible de $G$ sur $L$. On a un isomorphisme de $D\left(\mathbb{Z}_{p}^{s}, L\right) \widehat{\otimes}_{L} D\left(G_{0}, L\right)$-modules de type fini

$$
D\left(\mathbb{Z}_{p}^{s} \times G_{0}, L\right) \otimes_{\mathcal{O}_{L} \llbracket \mathbb{Z}_{p}^{s} \times G_{0} \rrbracket} \Pi^{\prime} \simeq\left(R^{\text {rig }} \widehat{\otimes}_{L} D\left(G_{0}, L\right)\right) \otimes_{R \llbracket G_{0} \rrbracket} \Pi^{\prime} .
$$

Démonstration. Soit $I$ le noyau du morphisme $\mathcal{O}_{L} \llbracket \mathbb{Z}_{p}^{s} \rrbracket \rightarrow R$. D'après le Lemme 5.1 en appendice, on a une suite exacte courte (topologique) d'espaces de Fréchet sur $L$

$$
0 \rightarrow D\left(\mathbb{Z}_{p}^{s}, L\right) I \rightarrow D\left(\mathbb{Z}_{p}^{s}, L\right) \rightarrow R^{\text {rig }} \rightarrow 0 .
$$

De plus par [55, Thm. 4.11] $D\left(\mathbb{Z}_{p}^{s}, L\right)$ est (fidèlement) plat sur $L \otimes_{\mathcal{O}_{L}} \mathcal{O}_{L} \llbracket \mathbb{Z}_{p}^{s} \rrbracket$, d'où un isomorphisme d'espaces de Fréchet $D\left(\mathbb{Z}_{p}^{s}, L\right) \otimes_{\mathcal{O}_{L} \llbracket \mathbb{Z}_{p}^{s} \rrbracket} I \simeq D\left(\mathbb{Z}_{p}^{s}, L\right) I$. Comme $D\left(G_{0}, L\right)$ est un espace de Fréchet sur $L$, le foncteur $D\left(G_{0}, L\right) \widehat{\otimes}_{L}-$ préserve les suites exactes courtes d'espaces de Fréchet sur $L$ (cf. par exemple [58, Lem. 4.13]), d'où une suite exacte d'espaces de Fréchet

$$
0 \rightarrow D\left(G_{0}, L\right) \widehat{\otimes}_{L}\left(D\left(\mathbb{Z}_{p}^{s}, L\right) I\right) \rightarrow D\left(G_{0}, L\right) \widehat{\otimes}_{L} D\left(\mathbb{Z}_{p}^{s}, L\right) \rightarrow D\left(G_{0}, L\right) \widehat{\otimes}_{L} R^{\text {rig }} \rightarrow 0
$$

et un isomorphisme

$$
D\left(G_{0}, L\right) \widehat{\otimes}_{L}\left(D\left(\mathbb{Z}_{p}^{s}, L\right) I\right) \simeq\left(D\left(G_{0}, L\right) \widehat{\otimes}_{L} D\left(\mathbb{Z}_{p}^{s}, L\right)\right) \otimes_{\mathcal{O}_{L} \llbracket \mathbb{Z}_{p}^{s} \rrbracket} I
$$

(rappelons que $I$ est un $\mathcal{O}_{L} \llbracket \mathbb{Z}_{p}^{s} \rrbracket$-module de type fini). Comme on a un isomorphisme $D\left(G_{0} \times \mathbb{Z}_{p}^{s}, L\right) \simeq D\left(G_{0}, L\right) \widehat{\otimes}_{L} D\left(\mathbb{Z}_{p}^{s}, L\right)$, on obtient une suite exacte courte

$$
0 \rightarrow D\left(G_{0} \times \mathbb{Z}_{p}^{s}, L\right) \otimes_{\mathcal{O}_{L} \llbracket \mathbb{Z}_{p}^{s} \rrbracket} I \rightarrow \underset{18}{D\left(G_{0} \times \mathbb{Z}_{p}^{s}, L\right) \rightarrow D\left(G_{0}, L\right) \widehat{\otimes}_{L} R^{\text {rig }} \rightarrow 0}
$$


et donc un isomorphisme $D\left(G_{0} \times \mathbb{Z}_{p}^{s}, L\right) \otimes_{\mathcal{O}_{L} \llbracket \mathbb{Z}_{p}^{s} \rrbracket} R \simeq D\left(G_{0}, L\right) \widehat{\otimes}_{L} R^{\text {rig }}$. Avec l'isomorphisme (évident) $R \otimes_{\mathcal{O}_{L} \llbracket \mathbb{Z}_{p}^{s} \rrbracket} \mathcal{O}_{L} \llbracket \mathbb{Z}_{p}^{s} \times G_{0} \rrbracket \cong R \llbracket G_{0} \rrbracket$, on en déduit donc des isomorphismes de $D\left(\mathbb{Z}_{p}^{s} \times G_{0}, L\right)$-modules (de type fini)

$$
\begin{aligned}
& D\left(\mathbb{Z}_{p}^{s} \times G_{0}, L\right) \otimes_{\mathcal{O}_{L} \llbracket \mathbb{Z}_{p}^{s} \times G_{0} \rrbracket} \Pi^{\prime} \simeq\left(D\left(\mathbb{Z}_{p}^{s} \times G_{0}, L\right) \otimes_{\mathcal{O}_{L} \llbracket \mathbb{Z}_{p}^{s} \times G_{0} \rrbracket} R \llbracket G_{0} \rrbracket\right) \otimes_{R \llbracket G_{0} \rrbracket} \Pi^{\prime} \\
\simeq & \left(D\left(\mathbb{Z}_{p}^{s} \times G_{0}, L\right) \otimes_{\mathcal{O}_{L} \llbracket \mathbb{Z}_{p}^{s} \times G_{0} \rrbracket}\left(R \otimes_{\mathcal{O}_{L} \llbracket \mathbb{Z}_{p}^{s} \rrbracket} \mathcal{O}_{L} \llbracket \mathbb{Z}_{p}^{s} \times G_{0} \rrbracket\right)\right) \otimes_{R \llbracket G_{0} \rrbracket} \Pi^{\prime} \\
\simeq & \left(D\left(\mathbb{Z}_{p}^{s} \times G_{0}, L\right) \otimes_{\mathcal{O}_{L} \llbracket \mathbb{Z}_{p}^{s} \rrbracket} R\right) \otimes_{R \llbracket G_{0} \rrbracket} \Pi^{\prime} \\
\simeq & \left(R^{\mathrm{rig}} \widehat{\otimes}_{L} D\left(G_{0}, L\right)\right) \otimes_{R \llbracket G_{0} \rrbracket} \Pi^{\prime} .
\end{aligned}
$$

On a donc un isomorphisme d'espaces de Fréchet nucléaires sur $L$

$$
\left(\Pi^{R-\mathrm{an}}\right)^{\prime} \simeq\left(R^{\mathrm{rig}} \widehat{\otimes}_{L} D\left(G_{0}, L\right)\right) \otimes_{R \llbracket G_{0} \rrbracket} \Pi^{\prime} .
$$

qui montre bien par bidualité ([53, Cor. 1.4]) que le sous-espace des vecteurs localement $R$-analytiques de $\Pi$ ne dépend pas du choix de la présentation de $R$.

On termine cette section avec la définition suivante. Si $J \subset R$ est un idéal et $\Pi$ une représentation de Banach $R$-admissible de $G$, on note $\Pi[J]$ le sous- $L$-espace vectoriel des $v \in \Pi$ tels que $J \cdot v=0$ muni de la topologie induite. Si $J$ est engendré par un seul élément $r \in R$, en dualisant la suite exacte $0 \rightarrow \Pi[J] \rightarrow \Pi \stackrel{r}{\rightarrow} \Pi$ (qui reste exacte car $\Pi$ est une représentation admissible de $\mathbb{Z}_{p}^{s} \times G$ ) on obtient un isomorphisme

$$
\Pi[J]^{\prime} \simeq \Pi^{\prime} / J \Pi^{\prime}
$$

compatible à $G$ et $R$. Par une récurrence immédiate (car $J$ est de type fini), on en déduit ce même isomorphisme pour tout idéal $J$. En particulier on voit que $\Pi[J]$ est une représentation $R / J$-admissible de $G$.

3.2. La variété de Hecke-Taylor-Wiles. On fixe $\mathfrak{m}$ un idéal maximal automorphe non Eisenstein de niveau modéré $U^{p}$ de $\mathbb{T}^{S}$ tel que $\mathbb{T}^{S} / \mathfrak{m}=k_{L}$ comme au $\S 2.4$, et on note simplement $\bar{\rho}$ au lieu de $\bar{\rho}_{\mathfrak{m}}$.

On fixe $g \geq 1$ un entier (pour l'instant quelconque) et on note $R_{\bar{\rho}_{\tilde{v}}}^{\bar{\square}}$ le quotient maximal

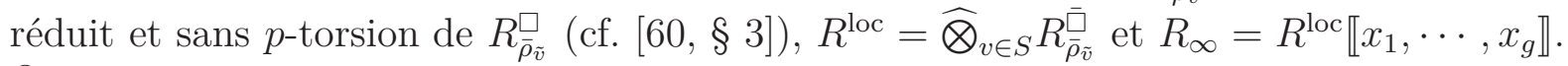
On pose aussi

$$
S_{\infty}=\mathcal{O}_{L} \llbracket y_{1}, \cdots, y_{q} \rrbracket
$$

où $q=g+\left[F^{+}: \mathbb{Q}\right] \frac{n(n-1)}{2}+|S| n^{2}$ et on note $\mathfrak{a}=\left(y_{1}, \cdots, y_{q}\right) \subset S_{\infty}$. Quitte à rapetisser le niveau modéré $U^{p}$ en des places totalement décomposées (et à augmenter $S$ en conséquence), on suppose que pour tout $h \in G\left(\mathbb{A}_{F^{+}}^{\infty}\right)$ il vérifie

$$
G\left(F^{+}\right) \cap\left(h U^{p} K_{p} h^{-1}\right)=\{1\} .
$$

Notons que la $\mathcal{O}_{L}$-algèbre $\mathbb{T}^{S}\left(U^{p} U_{p}, L\right)_{\mathfrak{m}}$ du $\S 2.4$ est réduite (et sans $p$-torsion) pour tout sous-groupe ouvert compact $U_{p}$ de $G_{p}$ : cela se déduit de la semi-simplicité de la $G\left(\mathbb{A}_{F^{+}}^{\infty}\right)$-représentation $\lim _{U} S(U, L) \otimes_{L} \overline{\mathbb{Q}}_{p}$ où la limite inductive est prise sur les sousgroupes compacts ouverts $U$ de $G\left(\mathbb{A}_{F^{+}}^{\infty}\right)$ (cf. par exemple [21, Prop. 3.3.2]). En particulier l'action de $R_{\bar{\rho}, S}$ sur $\widehat{S}\left(U^{p}, L\right)_{\mathfrak{m}}$ se factorise par $R_{\bar{\rho}, S} \rightarrow R_{\bar{\rho}, \mathcal{S}}$ où $R_{\bar{\rho}, \mathcal{S}}$ est l'anneau de déformation associé au problème de déformation (avec les notations de $[21, \S 2.3]$ )

$$
\mathcal{S}=\left(F / F^{+}, S, \widetilde{S}, \mathcal{O}_{L}, \bar{\rho}, \varepsilon^{1-n} \delta_{F / F^{+}}^{n},\left\{R_{\bar{\rho}_{\tilde{v}}}^{\overline{\bar{v}}}\right\}_{v \in S}\right)
$$


avec $\widetilde{S}=\{\widetilde{v} \mid v \in S\}$ et $\delta_{F / F^{+}}$le caractère quadratique de $\operatorname{Gal}\left(\bar{F} / F^{+}\right)$associé à l'extension $F / F^{+}$. Notre problème global de déformations n'est pas cadré, l'anneau $R_{\bar{\rho}, \mathcal{S}}$ est donc ici l'anneau noté $R_{\mathcal{S}}^{\text {univ }}$ dans [60].

Rappelons qu'une représentation continue $R_{\infty}$-admissible de $G_{p}$ sur $L$ est dite unitaire si pour un choix de norme la boule unité est stable par $G_{p}$ et par $R_{\infty}$. Le théorème suivant est essentiellement contenu dans les résultats de $[15, \S 2]$.

Théorème 3.4. Supposons $p \geq 2 n+2$ et que $\bar{\rho}$ reste absolument irréductible après restriction à $G_{F\left(\zeta_{p}\right)}$ où $\zeta_{p}$ est une racine primitive p-ième de l'unité. Il existe un entier $g \geq 1$ tel que l'on ait une représentation continue $R_{\infty}$-admissible unitaire $\Pi_{\infty}$ de $G_{p}$ sur $L$ et des morphismes de $\mathcal{O}_{L}$-algèbres locales $S_{\infty} \rightarrow R_{\infty}$ et $R_{\infty} \rightarrow R_{\bar{\rho}, \mathcal{S}}$ vérifiant les propriétés suivantes

(i) il existe une boule unité $\Pi_{\infty}^{0} \subset \Pi_{\infty}$ stable par $G_{p}$ et $R_{\infty}$ telle que

$$
M_{\infty}=\left(\Pi_{\infty}^{0}\right)^{\prime}=\operatorname{Hom}_{\mathcal{O}_{L}}\left(\Pi_{\infty}^{0}, \mathcal{O}_{L}\right)
$$

est un $S_{\infty} \llbracket K_{p} \rrbracket$-module projectif de type fini (via $S_{\infty} \rightarrow R_{\infty}$ );

(ii) il existe un isomorphisme $R_{\infty} / \mathfrak{a} R_{\infty} \simeq R_{\bar{\rho}, \mathcal{S}}$ de $\mathcal{O}_{L}$-algèbres locales noethériennes complètes et un isomorphisme de représentations continues $R_{\infty} / \mathfrak{a} R_{\infty}$-admissibles unitaires de $G_{p}$ sur $L$

$$
\Pi_{\infty}[\mathfrak{a}] \simeq \widehat{S}\left(U^{p}, L\right)_{\mathfrak{m}}
$$

Démonstration. Une telle représentation continue est essentiellement construite dans [15, Cor. 2.9] en prenant pour $\Pi_{\infty}$ le dual continu du $R_{\infty} \llbracket K_{p} \rrbracket$-module de type fini $M_{\infty}$ de loc. cit. (cf. [52, Thm. 3.5], on utilise comme au $\S 3.1$ que $R_{\infty} \llbracket K_{p} \rrbracket$ est un quotient de l'algèbre d'Iwasawa de $\mathbb{Z}_{p}^{s} \times K_{p}$ pour $s$ convenable). La seule différence avec $[15, \S 2]$ est qu'il faut remplacer la place $\mathfrak{p}$ de $F^{+}$fixée au-dessus de $p$ par l'ensemble $S_{p}$ (i.e. toutes les places au-dessus de $p$ ) et l'ensemble $T=S_{p} \cup\left\{v_{1}\right\}$ (où $v_{1}$ est une place hors $p$ décomposée particulière dont le but est d'obtenir des propriétés de multiplicité 1 dont nous n'avons pas besoin, cf. $[15, \S 2.3]$ ) par l'ensemble $T=S$. En remplaçant donc le problème de déformation $\mathcal{S}_{Q_{N}}$ de $[15, \S 2.5]$ par le problème de déformation (en adaptant les notations de loc. cit.)

$$
\left(F / F^{+}, S \cup Q_{N}, \widetilde{S} \cup \widetilde{Q}_{N}, \mathcal{O}_{L}, \bar{\rho}, \varepsilon^{1-n} \delta_{F / F^{+}}^{n},\left\{R_{\bar{\rho}_{\tilde{v}}}^{\overline{\bar{v}}}\right\}_{v \in S} \cup\left\{R_{\bar{\rho}_{\tilde{v}}}^{\bar{\psi}_{\tilde{v}}}\right\}_{v \in Q_{N}}\right),
$$

une preuve analogue à celle de [15, Cor. 2.9] donne le $M_{\infty}$ recherché.

La représentation continue $\Pi_{\infty}$ du Théorème 3.4 est donc à la fois une représentation $R_{\infty}$-admissible de $G_{p}$ et une représentation $S_{\infty}$-admissible.

Rappelons que nous avons fixé au $\S 2.4$ un sous-groupe de Borel $B_{p} \subset G_{p}$ de tore diagonal $T_{p}$. En appliquant le foncteur $J_{B_{p}}$ défini par Emerton dans [26, Def. 3.4.5] à la représentation $\Pi^{R_{\infty} \text {-an }}$ de $G_{p}$ (cf. Remarque 3.5 ci-dessous) et en prenant le dual continu, on obtient un $R_{\infty}^{\mathrm{rig}} \widehat{\otimes}_{L} \mathcal{O}\left(\widehat{T}_{p, L}\right)$-module coadmissible $J_{B_{p}}\left(\Pi_{\infty}^{R_{\infty}-\text { an }}\right)^{\prime}$ au sens de [55, $\S 3]$ (voir aussi $[25, \S 1.2]$ ), qui lui-même correspond à un unique faisceau cohérent $\mathcal{M}_{\infty}$ sur $\operatorname{Spf}\left(R_{\infty}\right)^{\text {rig }} \times_{L} \widehat{T}_{p, L}$ (au sens de $\left.[9, \S 9.4]\right)$ tel que

$$
\Gamma\left(\operatorname{Spf}\left(R_{\infty}\right)^{\mathrm{rig}} \times_{L} \widehat{T}_{p, L}, \mathcal{M}_{\infty}\right) \simeq J_{B_{p}}\left(\Pi_{\infty}^{R_{\infty}-\mathrm{an}}\right)^{\prime}
$$

(voir encore par exemple $[55, \S 3]$ et la discussion qui précède le Lemme 3.3). 
Remarque 3.5. Précisons un peu le point précédent. À proprement parler, Emerton ne construit le foncteur $J_{P}$ que pour une représentation localement analytique du groupe des $\mathbb{Q}_{p}$-points d'un sous-groupe parabolique $P$ d'un groupe réductif défini sur $\mathbb{Q}_{p}$. Mais si l'on fixe une présentation $\mathcal{O}_{L} \llbracket \mathbb{Z}_{p}^{s} \rrbracket \rightarrow R_{\infty}$ de l'algèbre $R_{\infty}$ comme au $\S 3.1$, on peut voir $\Pi_{\infty}^{R_{\infty} \text {-an }}$ comme une représentation localement analytique du groupe $\mathbb{Z}_{p}^{s} \times G_{p}$ (qui ne dépend pas de la présentation, cf. § 3.1). Il ne s'agit pas du groupe des $\mathbb{Q}_{p}$-points d'un groupe réductif, mais il n'est pas difficile de vérifier que la construction de $J_{B_{p}}$ s'étend au groupe $\mathbb{Z}_{p}^{s} \times G_{p}$.

On suppose que l'extension $L$ de $\mathbb{Q}_{p}$ est suffisamment grande de telle sorte que, pour tout $v \in S$, les composantes irréductibles du schéma $\operatorname{Spec}\left(R_{\overline{\bar{\rho}} \tilde{\bar{v}}}^{\bar{\square}}[1 / p]\right)$ soient géométriquement irréductibles (i.e. $C \times_{L} \overline{\mathbb{Q}}_{p}$ reste un schéma irréductible pour toute composante irréductible $C$ de $\left.\operatorname{Spec}\left(R_{\overline{\bar{\rho}}_{\tilde{v}}}^{\bar{\square}}[1 / p]\right)\right)$.

Si $X$ est un espace analytique rigide et $\mathcal{M}$ un faisceau cohérent sur $X$, on appelle support schématique de $\mathcal{M}$ la variété analytique rigide dont l'espace sous-jacent est le fermé analytique $\left\{x \in X \mid \mathcal{M}_{x} \neq 0\right\}$ de $X$ (cf. ([9, § 9.5.2 Prop.4])) et le faisceau structural est donné par $\mathcal{O}_{X} / \mathcal{I}$ où $\mathcal{I} \subset \mathcal{O}_{X}$ est le faisceau cohérent d'idéaux annulateurs de $\mathcal{M}$.

Définition 3.6. On note $X_{p}(\bar{\rho}) \subset \operatorname{Spf}\left(R_{\infty}\right)^{\mathrm{rig}} \times_{L} \widehat{T}_{p, L}$ le support schématique du faisceau cohérent $\mathcal{M}_{\infty}$. On note également $X_{p}(\bar{\rho})^{\mathrm{red}}$ la nilréduction de $X_{p}(\bar{\rho})$, au sens de $[9, \mathrm{p}$. 389], que l'on appelle variété de Hecke-Taylor-Wiles.

Nous prouverons en fait plus loin que $X_{p}(\bar{\rho})$ est un espace analytique réduit, de sorte que $X_{p}(\bar{\rho})=X_{p}(\bar{\rho})^{\text {red }}$.

Il sera utile dans la suite de désigner par $\mathfrak{X}_{\bar{\rho}^{l}}^{\square}$ 'espace analytique rigide $\operatorname{Spf}\left(\widehat{\bigotimes}_{v \in S \backslash S_{p}} R_{\bar{\rho}_{\tilde{v}}}^{\overline{\bar{v}}}\right)^{\text {rig }}$ et par $\mathfrak{X}_{\bar{\rho}_{p}}^{\square}$ l'espace $\operatorname{Spf}\left(\widehat{\bigotimes}_{v \in S_{p}} R_{\bar{\rho}_{\tilde{v}}}^{\overline{\bar{v}}}\right)^{\text {rig }}$. On note $\mathbb{U}$ la boule ouverte de rayon 1 , autrement dit l'espace analytique rigide $\operatorname{Spf}\left(\mathcal{O}_{L} \llbracket T \rrbracket\right)^{\text {rig. }}$. On a $\operatorname{Spf}\left(R_{\infty}\right)^{\text {rig }} \simeq \mathfrak{X}_{\bar{\rho}_{p}}^{\square} \times \mathfrak{X}_{\bar{\rho}^{p}}^{\square} \times \mathbb{U}^{g}$ par [38, Prop. 7.2.4.g)].

Si $V$ est une représentation localement analytique de $T_{p}$ et $\delta$ est un caractère localement analytique de $T_{p}$, on note $V^{T_{p}=\delta}$ le sous-espace de $V$ où l'action de $T_{p}$ se factorise par $\delta$.

Proposition 3.7. Soit $x=(y, \delta) \in\left(\operatorname{Spf}\left(R_{\infty}\right)^{\text {rig }} \times \widehat{T}_{p, L}\right)(L)$. Notons $\mathfrak{p}_{x}$ l'idéal maximal de $R_{\infty}[1 / p]$ correspondant au point $y$. Alors $x \in X_{p}(\bar{\rho})$ si et seulement si $\left(J_{B_{p}}\left(\Pi_{\infty}\left[\mathfrak{p}_{x}\right]^{\text {an }}\right)\right)^{T_{p}=\delta}$ est non nul.

Démonstration. De même qu'en [27, Prop. 2.3.3(iii)], la fibre de $\mathcal{M}_{\infty}$ en $x$ est duale de

$$
\left(J_{B_{p}}\left(\Pi_{\infty}^{R_{\infty}-\mathrm{an}}\right)\left[\mathfrak{p}_{x}\right]\right)^{T_{p}=\delta} .
$$

Pour conclure on utilise l'isomorphisme $J_{B_{p}}\left(\Pi_{\infty}\left[\mathfrak{p}_{x}\right]^{\text {an }}\right) \simeq J_{B_{p}}\left(\Pi_{\infty}^{R_{\infty}-\text { an }}\right)\left[\mathfrak{p}_{x}\right]$ qui résulte de l'exactitude du foncteur $\Pi \mapsto \Pi^{\text {an }}$ (qui est une conséquence de [55, Thm. 7.1]), de l'exactitude à gauche du foncteur $J_{B_{p}}([26$, Lem. 3.4.7]) et de l'isomorphisme (3.3).

\subsection{Premières propriétés.}

Proposition 3.8. L'inclusion $\Pi_{\infty}^{R_{\infty} \text {-an }} \subset \Pi_{\infty}^{S_{\infty} \text {-an }}$ provenant du morphisme $S_{\infty} \rightarrow R_{\infty}$ est une égalité.

Démonstration. Notons $A$ l'image de $R_{\infty} \llbracket K_{p} \rrbracket$ dans $\operatorname{End}\left(M_{\infty}\right)$. Fixons $e_{1}, \cdots, e_{m}$ une famille de générateurs du $S_{\infty} \llbracket K_{p} \rrbracket$-module de type fini $M_{\infty}$ (voir (i) du Théorème 3.4 ). 
L'application $f \mapsto\left(f\left(e_{1}\right), \cdots, f\left(e_{m}\right)\right)$, où $f \in A \subset \operatorname{End}\left(M_{\infty}\right)$, permet d'identifier $A$ à un sous- $S_{\infty} \llbracket K_{p} \rrbracket$-module de $M_{\infty}^{m}$. L'isomorphisme $S_{\infty} \llbracket K_{p} \rrbracket \simeq \mathcal{O}_{L} \llbracket \mathbb{Z}_{p}^{q} \times K_{p} \rrbracket$ ainsi que [46, Prop. V.2.2.4] (combiné au fait que $K_{p}$ contient un pro-p-sous-groupe analytique d'indice fini) montrent que $S_{\infty} \llbracket K_{p} \rrbracket$ est un anneau noethérien. On en déduit que $A$ est un $S_{\infty} \llbracket K_{p} \rrbracket$ module de type fini. D'après le Lemme 5.2 en appendice appliqué à $S_{\infty} \llbracket K_{p} \rrbracket \rightarrow A$ et $R_{\infty} \llbracket K_{p} \rrbracket \rightarrow A$, on a $\left(S_{\infty} \llbracket K_{p} \rrbracket\right)^{\mathrm{rig}} \otimes_{S_{\infty} \llbracket K_{p} \rrbracket} A \simeq A^{\mathrm{rig}} \simeq R_{\infty} \llbracket K_{p} \rrbracket^{\text {rig }} \otimes_{R_{\infty} \llbracket K_{p} \rrbracket} A$, ce qui prouve, par (3.2) et [38, Prop. 7.2.4.g], que

$$
\begin{aligned}
\left(\Pi_{\infty}^{S_{\infty}-\mathrm{an}}\right)^{\prime} & \simeq\left(S_{\infty}^{\mathrm{rig}} \widehat{\otimes}_{L} D\left(K_{p}, L\right)\right) \otimes_{S_{\infty} \llbracket K_{p} \rrbracket} \Pi_{\infty}^{\prime} \\
& \simeq\left(S_{\infty} \llbracket K_{p} \rrbracket^{\mathrm{rig}} \otimes_{S_{\infty} \llbracket K_{p} \rrbracket} A\right) \otimes_{A} \Pi_{\infty}^{\prime} \\
& \simeq A^{\mathrm{rig}} \otimes_{A} \Pi_{\infty}^{\prime} \\
& \simeq R_{\infty} \llbracket K_{p} \rrbracket^{\mathrm{rig}} \otimes_{R_{\infty} \llbracket K_{p} \rrbracket} \Pi_{\infty}^{\prime} \\
& \simeq\left(R_{\infty}^{\mathrm{rig}} \widehat{\otimes}_{L} D\left(K_{p}, L\right)\right) \otimes_{R_{\infty} \llbracket K_{p} \rrbracket} \Pi_{\infty}^{\prime} \\
& \simeq\left(\Pi_{\infty}^{R_{\infty}-\mathrm{an}}\right)^{\prime} .
\end{aligned}
$$

Notons $\mathcal{W}_{\infty}$ l'espace $\operatorname{Spf}\left(S_{\infty}\right)^{\text {rig }} \times \widehat{T}_{p, L}^{0}$ qui joue ici le même rôle que l'espace des poids pour les variétés de Hecke. On définit en effet une application poids $\omega_{X}$ de $X_{p}(\bar{\rho})$ dans $\mathcal{W}_{\infty}$ comme la composée de l'inclusion de $X_{p}(\bar{\rho})$ dans $\operatorname{Spf}\left(R_{\infty}\right)^{\text {rig }} \times \widehat{T}_{p, L}$ avec l'application de $\operatorname{Spf}\left(R_{\infty}\right)^{\text {rig }} \times \widehat{T}_{p, L}$ vers $\operatorname{Spf}\left(S_{\infty}\right)^{\text {rig }} \times \widehat{T}_{p, L}^{0}=\mathcal{W}_{\infty}$ déduite de la structure de $S_{\infty}$-algèbre de $R_{\infty}$ et de la restriction $\widehat{T}_{p, L} \rightarrow \widehat{T}_{p, L}^{0}$. Soit $z \in T_{p}$ un élément tel que $|\alpha(z)|_{p}<1$ pour toute racine positive $\alpha$, relativement à $B_{p}$, du groupe $G \times_{F^{+}}\left(F^{+} \otimes_{\mathbb{Q}} \mathbb{Q}_{p}\right)$. On peut par exemple choisir $z=\left(z_{v}\right)_{v \in S_{p}} \in \prod_{v \in S_{p}} T_{v}$, où $z_{v}$ désigne l'élément diagonal $\left(\varpi_{v}^{n-1}, \ldots, \varpi_{v}, 1\right)$. Il définit une application de restriction $\operatorname{Spf}\left(S_{\infty}\right)^{\mathrm{rig}} \times \widehat{T}_{p, L} \rightarrow \mathcal{W}_{\infty} \times \mathbb{G}_{m, L}$, déduite de l'inclusion $z^{\mathbb{Z}} \subset T_{p}$ et de l'isomorphisme d'espaces analytiques rigides $\widehat{\mathbb{Z}} \simeq \mathbb{G}_{m}$. On note alors

$$
f: \operatorname{Spf}\left(R_{\infty}\right)^{\mathrm{rig}} \times \widehat{T}_{p, L} \longrightarrow \mathcal{W}_{\infty} \times \mathbb{G}_{m, L}
$$

sa composée avec le morphisme $\operatorname{Spf}\left(R_{\infty}\right)^{\text {rig }} \times \widehat{T}_{p, L} \rightarrow \operatorname{Spf}\left(S_{\infty}\right)^{\text {rig }} \times \widehat{T}_{p, L}$ provenant de la structure de $S_{\infty}$-algèbre de $R_{\infty}$. Finalement on note $g$ la projection $\mathcal{W}_{\infty} \times \mathbb{G}_{m, L} \rightarrow \mathcal{W}_{\infty}$.

Notons $Y$ le sous-groupe fermé de $T_{p}$ engendré par $T_{p}^{0}$ et $z$. Ce sous-groupe vérifie les conditions de la discussion précédant [26, Prop. 3.2.27]. On déduit alors de loc. cit., de [26, Prop. 3.2.23] et de la Proposition 5.3 de l'appendice que $J_{B_{p}}\left(\Pi_{\infty}^{R_{\infty} \text {-an }}\right)^{\prime}$ est également un $\mathcal{O}\left(\mathcal{W}_{\infty} \times \mathbb{G}_{m, L}\right)$-module coadmissible en identifiant $\mathcal{W}_{\infty} \times \mathbb{G}_{m, L}$ à $\operatorname{Spf}\left(S_{\infty}\right)^{\text {rig }} \times \hat{Y}$. Comme précédemment, il s'agit de l'espace des sections globales d'un faisceau cohérent $\mathcal{N}_{\infty}$ de $\mathcal{O}_{\mathcal{W}_{\infty} \times \mathbb{G}_{m, L}}$-modules. Notons $Z_{z}(\bar{\rho})$ le support schématique de ce faisceau cohérent dans $\mathcal{W}_{\infty} \times \mathbb{G}_{m, L}$. L'application $f$ de $X_{p}(\bar{\rho})$ dans $\mathcal{W}_{\infty} \times \mathbb{G}_{m, L}$ se factorise par $Z_{z}(\bar{\rho})$, on note encore $f$ l'application $f: X_{p}(\bar{\rho}) \rightarrow Z_{z}(\bar{\rho})$ obtenue ainsi. De même, on note encore $g$ la restriction de $g$ à $Z_{z}(\bar{\rho})$. On obtient ainsi un diagramme commutatif

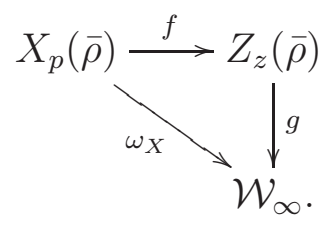

Les espaces $X_{p}(\bar{\rho})$ et $Z_{z}(\bar{\rho})$ sont quasi-Stein puisque ce sont des fermés analytiques de $\operatorname{Spf}\left(R_{\infty}\right) \times_{L} \widehat{T}_{p, L}$ et $\mathcal{W}_{\infty} \times_{L} \mathbb{G}_{m, L}$ qui sont eux-mêmes quasi-Stein. 
Rappelons qu'une hypersurface de Fredholm de $\mathcal{W}_{\infty} \times \mathbb{G}_{m, L}$ est un fermé analytique de la forme $Z(F)$ pour $F \in 1+T \mathcal{O}\left(\mathcal{W}_{\infty}\right)\{\{T\}\}$, où

$$
Z(F)=\left\{(x, t) \in \mathcal{W}_{\infty} \times \mathbb{G}_{m, L} \mid F\left(x, t^{-1}\right)=0\right\},
$$

et $\mathcal{O}\left(\mathcal{W}_{\infty}\right)\{\{T\}\}$ désigne l'anneau des séries $\sum_{n \geq 0} a_{n} T^{n}$ convergeant sur $\mathcal{W}_{\infty} \times \mathbb{A}_{L}^{1}$ (dans $\left[24, \S 4\right.$, la notion d'hypersurface de Fredholm est définie en remplaçant $\mathbb{G}_{m, L}$ par $\mathbb{A}_{L}^{1}$, mais cela ne change rien puisque l'ensemble des zéros d'une telle série $F$ est contenu dans l'ouvert $\mathcal{W}_{\infty} \times \mathbb{G}_{m, L}$ de $\left.\mathcal{W}_{\infty} \times \mathbb{A}_{L}^{1}\right)$.

Lemme 3.9. Le fermé analytique sous-jacent à $Z_{z}(\bar{\rho})$ est une hypersurface de Fredholm de $\mathcal{W}_{\infty} \times \mathbb{G}_{m, L}$. De plus, il existe un recouvrement admissible $\left(U_{i}^{\prime}\right)_{i \in I}$ de $Z_{z}(\bar{\rho})$ par des affinoïdes $U_{i}^{\prime}$ tels que $g$ induise une application finie surjective de $U_{i}^{\prime}$ sur un ouvert affinoïde $W_{i}$ de $\mathcal{W}_{\infty}$ et que $U_{i}^{\prime}$ soit une composante connexe de $g^{-1}\left(W_{i}\right)$. Enfin, pour un tel recouvrement, pour tout $i \in I$, le module $\Gamma\left(U_{i}^{\prime}, \mathcal{N}_{\infty}\right)$ est un $\mathcal{O}_{\mathcal{W}_{\infty}}\left(W_{i}\right)$-module projectif de type fini.

Démonstration. D'après la Proposition 5.3 de l'appendice, il existe un recouvrement admissible de $\mathcal{W}_{\infty}$ par des ouverts affinoïdes $U_{1} \subset U_{2} \subset \cdots \subset U_{h} \subset \cdots$, des $A_{h}=\mathcal{O}_{\mathcal{W}_{\infty}}\left(U_{h}\right)$ modules de Banach de type $(\operatorname{Pr}), V_{h}$, munis d'endomorphismes $A_{h}$-compacts $z_{h}$, un sousgroupe compact ouvert $N_{0} \subset N_{p}$ et un diagramme commutatif

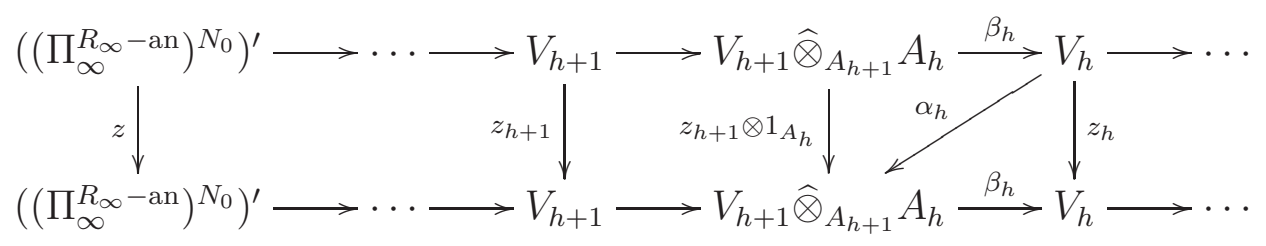

de sorte que les lignes horizontales induisent un isomorphisme topologique

$$
\left(\left(\Pi_{\infty}^{R_{\infty}-\mathrm{an}}\right)^{N_{0}}\right)^{\prime} \simeq \lim _{h} V_{h}
$$

(en ce qui concerne les notions de $A_{h}$-module de Banach de type $(P r)$ et d'endomorphisme $A_{h}$-compact, nous renvoyons à $\left.[12, \S 2]\right)$.

Notons $F_{h}$ la série caractéristique de $z_{h}$ (cf. par exemple la fin de $[12, \S 2]$ ). Il s'agit d'un élément de $A_{h}\{\{T\}\}$. Les factorisations $z_{h}=\beta_{h} \circ \alpha_{h}$ et $z_{h+1} \otimes 1_{A_{h}}=\alpha_{h} \circ \beta_{h}$, ainsi que [12, Lemma 2.12] montrent que $F_{h}$ coïncide avec la série caractéristique de $z_{h+1} \otimes 1_{A_{h}}$. On déduit alors de [12, Lemma 2.13] que l'image de $F_{h+1}$ dans $A_{h}\{\{T\}\}$ coïncide avec $F_{h}$. On note $F$ l'élément $\left(F_{h}\right)_{h \geq 1}$ de $\mathcal{O}\left(\mathcal{W}_{\infty}\right)\{\{T\}\}$.

Montrons que le fermé analytique sous-jacent à $Z_{z}(\bar{\rho})$ est exactement l'ensemble des points $(y, \lambda) \in \mathcal{W}_{\infty} \times \mathbb{G}_{m, L}$ tels que $F\left(y, \lambda^{-1}\right)=0$. Fixons un tel $x=(y, \lambda) \in \mathcal{W}_{\infty} \times \mathbb{G}_{m, L}$ et $h$ tel que $y \in U_{h}$. Comme l'espace $\mathcal{W}_{\infty} \times \mathbb{G}_{m, L}$ est quasi-Stein, [40, Satz 2.4.3] montre que la fibre de $\mathcal{N}_{\infty}$ en $x$ est non nulle si et seulement si $\Gamma\left(U_{h} \times \mathbb{G}_{m, L}, \mathcal{N}_{\infty}\right) \otimes_{\mathcal{O}\left(U_{h} \times \mathbb{G}_{m, L}\right)} k(x) \neq 0$, où $k(x)$ désigne le corps résiduel de $W_{\infty} \times_{L} \mathbb{G}_{m, L}$ en $x$. Les lemmes 5.5 et 5.6 en appendice montrent que l'on a un isomorphisme

$$
\Gamma\left(U_{h} \times_{L} \mathbb{G}_{m, L}, \mathcal{N}_{\infty}\right) \simeq \lim _{h^{\prime} \geq h}\left(V_{h^{\prime}} \widehat{\otimes}_{A_{h^{\prime}}} A_{h}\right)
$$

On déduit alors de l'existence des flèches $\alpha_{h^{\prime}}$ et $\beta_{h^{\prime}}$, ainsi que du diagramme (3.5), que la fibre de $\mathcal{N}_{\infty}$ en $x$ est non nulle si et seulement si $V_{h} /\left(\left(z_{h}-\lambda\right) V_{h}+\mathfrak{p}_{y} V_{h}\right) \neq 0$, où $\mathfrak{p}_{y}$ est l'idéal maximal de $A_{h}$ associé au point $y$. Or d'après [51, Cor. 22.9], l'application $1-\lambda^{-1} z_{h}$ est un endomorphisme de Fredholm d'indice 0 de $V_{h} / \mathfrak{p}_{y} V_{h}$ ( $z_{h}$ étant un endomorphisme $A_{h}$-compact de $V_{h}$, il induit un endomorphisme compact de $\left.V_{h} / \mathfrak{p}_{y} V_{h}\right)$, ce qui signifie que 
son noyau et son conoyau sont de même dimension finie (cf. [51, §22] pour les notions d'endomorphisme de Fredholm et d'indice). Ainsi $(y, \lambda) \in X_{p}(\bar{\rho})$ si et seulement si le noyau de $1-\lambda^{-1} z_{h}$ agissant sur $V_{h} / \mathfrak{p}_{y} V_{h}$ est non nul, ce qui équivaut à demander que $\lambda^{-1}$ soit un zéro de la série caractéristique de $z_{h}$ sur $V_{h} / \mathfrak{p}_{y} V_{h}$ (voir [12, Prop. 3.2] par exemple). On conclut alors en remarquant que cette série caractéristique est l'image de $F_{h} \in A_{h}\{\{T\}\}$ dans $k(y)\{\{T\}\}$ par réduction modulo $\mathfrak{p}_{y}$ ([12, Lemma 2.13]).

D'après [12, Thm. 4.6], il existe un recouvrement admissible du fermé analytique de $Z_{z}(\bar{\rho})$ par des ouverts affinoïdes $U_{i}^{\prime}$ tels que l'application $g: Z_{z}(\bar{\rho}) \rightarrow \mathcal{W}_{\infty}$ induise une application finie surjective $U_{i}^{\prime} \rightarrow W_{i}$ avec $W_{i}$ un ouvert affinoïde $\operatorname{de} \mathcal{W}_{\infty}$ et $U_{i}^{\prime}$ soit une composante connexe de $g^{-1}\left(W_{i}\right)$. Fixons $i \in I$. La discussion des cinq premiers paragraphes de $[12, \S 5]$ montre alors que $U_{i}^{\prime}$ est un fermé analytique de $W_{i} \times_{L} \mathbb{G}_{m, L}$ défini par un idéal engendré par un polynôme $Q(T) \in 1+T \mathcal{O}_{\mathcal{W}_{\infty}}\left(W_{i}\right)[T]$ et tel que $F(T)=Q(T) S(T)$ où $S(T)$ est une série de Fredholm dans $1+T \mathcal{O}_{\mathcal{W}_{\infty}}\left(W_{i}\right)\{\{T\}\}$ telle que $(Q, S)=1$. On obtient ainsi, d'après le Lemme 5.5,

$$
\Gamma\left(U_{i}^{\prime}, \mathcal{N}_{\infty}\right)=\lim _{U_{h}^{\leftarrow} \supset W_{i}}\left(\left(V_{h} \widehat{\otimes}_{A_{h}\{\{T\}\}} \mathcal{O}_{\mathcal{W}_{\infty}}\left(W_{i}\right)\{\{T\}\}\right) / Q(T)\right)
$$

où $T$ agit sur $V_{h}$ via $z_{h}^{-1}$. Or on peut décomposer $V_{h} \widehat{\otimes}_{A_{h}} \mathcal{O}_{\mathcal{W}_{\infty}}\left(W_{i}\right)$ en une somme directe $N_{i} \oplus F_{i}$ où $N_{i}$ est un $\mathcal{O}_{\mathcal{W}_{\infty}}\left(W_{i}\right)$-module projectif de $\operatorname{rang} \operatorname{deg} Q$ sur lequel $Q^{*}\left(z_{h}\right)$ s'annule et $Q^{*}\left(z_{h}\right)$ est inversible sur $F_{i}$ (suivant [12, §3], $Q^{*}(T)=T^{\operatorname{deg} Q} Q\left(T^{-1}\right)$ ). Ainsi chaque terme du système projectif (3.6) est un $\mathcal{O}_{\mathcal{W}_{\infty}}\left(W_{i}\right)$-module projectif de $\operatorname{rang} \operatorname{deg} Q$ et l'existence des flèches $\alpha_{h}$ ainsi que le fait que $Q^{*}\left(z_{h}\right)$ soit inversible sur $F_{i}$ montrent que les flèches de transition du système (3.6) sont des isomorphismes. Ainsi $\Gamma\left(U_{i}^{\prime}, \mathcal{N}_{\infty}\right)$ est un $\mathcal{O}_{\mathcal{W}_{\infty}}\left(W_{i}\right)$-module projectif de type fini.

Proposition 3.10. Il existe un recouvrement admissible affinoïde $\left(U_{i}\right)_{i \in I}$ de $X_{p}(\bar{\rho})$ tel que pour tout $i$, il existe $W_{i}$ ouvert affinoïde de $\mathcal{W}_{\infty}$ tel que $\omega_{X}$ induise, en restriction à chaque composante irréductible de $U_{i}$, un morphisme fini surjectif sur $W_{i}$ et que $\mathcal{O}_{X_{p}(\bar{\rho})}\left(U_{i}\right)$ soit isomorphe à une $\mathcal{O}_{\mathcal{W}_{\infty}}\left(W_{i}\right)$-algèbre d'endomorphismes d'un $\mathcal{O}_{\mathcal{W}_{\infty}}\left(W_{i}\right)$-module projectif de rang fini.

Démonstration. On fixe $\left(U_{i}^{\prime}\right)_{i \in I}$ un recouvrement affinoïde admissible de $Z_{z}(\bar{\rho})$ comme dans le Lemme 3.9, on pose $U_{i}=f^{-1}\left(U_{i}^{\prime}\right)$ et $W_{i}=g\left(U_{i}^{\prime}\right)$. La famille $\left(U_{i}\right)_{i \in I}$ est alors un recouvrement ouvert admissible de $X_{p}(\bar{\rho})$. Nous allons prouver que chaque $U_{i}$ est affinoïde et que $\mathcal{O}_{X_{p}(\bar{\rho})}\left(U_{i}\right)$ est isomorphe à une sous- $\mathcal{O}_{\mathcal{W}_{\infty}}\left(W_{i}\right)$-algèbre de $\operatorname{End}_{\mathcal{O}_{W_{\infty}}\left(W_{i}\right)}\left(\Gamma\left(U_{i}^{\prime}, \mathcal{N}_{\infty}\right)\right)$. Ceci implique en particulier que $\mathcal{O}_{X_{p}(\bar{\rho})}\left(U_{i}\right)$ est un $\mathcal{O}_{\mathcal{W}_{\infty}}\left(W_{i}\right)$-module fini et que l'application $\mathcal{O}_{\mathcal{W}_{\infty}}\left(W_{i}\right) \rightarrow \mathcal{O}_{X_{p}(\bar{\rho})}\left(U_{i}\right)$ est injective. Comme un morphisme fini d'espaces rigides est un morphisme propre, en particulier fermé, on en déduit que $\omega_{X}$ induit une application finie surjective de $U_{i}$ sur $W_{i}$. La surjectivité en restriction à chaque composante irréductible est alors une conséquence de [16, Lem. 6.2.10].

Posons $M=J_{B_{p}}\left(\Pi_{\infty}^{R_{\infty}-\text { an }}\right)^{\prime}$. D'après le Lemme 5.6 en appendice, on a un isomorphisme entre $\mathcal{O}_{X_{p}(\bar{\rho})}\left(U_{i}\right)$ et $\mathcal{O}\left(X_{p}(\bar{\rho})\right) \widehat{\otimes}_{\mathcal{O}\left(Z_{z}(\bar{\rho})\right)} \mathcal{O}_{Z_{z}(\bar{\rho})}\left(U_{i}^{\prime}\right)$. On déduit donc du Lemme 5.5 un isomorphisme de $\mathcal{O}_{Z_{z}(\bar{\rho})}\left(U_{i}^{\prime}\right)$-modules

$$
\Gamma\left(U_{i}, \mathcal{M}_{\infty}\right) \simeq \mathcal{O}_{X_{p}(\bar{\rho})}\left(U_{i}\right) \widehat{\otimes}_{\mathcal{O}\left(X_{p}(\bar{\rho})\right)} M \simeq \Gamma\left(U_{i}^{\prime}, \mathcal{N}_{\infty}\right)
$$

Ainsi, $\Gamma\left(U_{i}, \mathcal{M}_{\infty}\right)$ est en particulier un $\mathcal{O}_{\mathcal{W}_{\infty}}\left(W_{i}\right)$-module projectif de type fini par le Lemme 3.9. Comme $X_{p}(\bar{\rho})$ est le support de $\mathcal{M}_{\infty}$, l'action de $\mathcal{O}_{X_{p}(\bar{\rho})}\left(U_{i}\right)$ sur $\Gamma\left(U_{i}, \mathcal{M}_{\infty}\right)$ est fidèle, en particulier $\mathcal{O}_{X_{p}(\bar{\rho})}\left(U_{i}\right)$ est isomorphe à une sous- $\mathcal{O}_{\mathcal{W}_{\infty}}\left(W_{i}\right)$-algèbre de l'algèbre des endomorphismes $\mathcal{O}_{W_{\infty}}\left(W_{i}\right)$-linéaires de $\Gamma\left(U_{i}, \mathcal{M}_{\infty}\right)$. 
Corollaire 3.11. L'espace $X_{p}(\bar{\rho})$ est équidimensionnel de dimension

$$
g+\left[F^{+}: \mathbb{Q}\right] \frac{n(n+1)}{2}+|S| n^{2}
$$

et n'a pas de composante immergée. De plus le morphisme $f: X_{p}(\bar{\rho}) \rightarrow Z_{z}(\bar{\rho})$ est fini et l'image d'une composante irréductible de $X_{p}(\bar{\rho})$ par $f$ est une composante irréductible de $Z_{z}(\bar{\rho})$.

Démonstration. Considérons un recouvrement de $X_{p}(\bar{\rho})$ par des ouverts affinoïdes $U$ comme dans la Proposition 3.10. Pour tout $U$, posons $V=\omega_{X}(U)$, alors $B=\mathcal{O}_{X_{p}(\bar{\rho})}(U)$ est une $\mathrm{A}=\mathcal{O}_{\mathcal{W}_{\infty}}(V)$-algèbre agissant fidèlement sur un $A$-module projectif de type fini $M$. Ceci implique que si $y$ est un point de $U$ d'image $x$ dans $V$, l'anneau $\widehat{B}_{y}$ est isomorphe à un sous- $\widehat{A}_{x}$-module d'un $\widehat{A}_{x}$-module libre de type fini (on utilise le fait que l'anneau $\widehat{A}_{x}$ est complet donc hensélien). Si p est un idéal premier associé de $\widehat{B}_{y}$, il existe par définition un plongement de $\widehat{B}_{y} / \mathfrak{p}$ dans $\widehat{B}_{y}$. Comme $\widehat{A}_{x}$ est intègre, on en déduit que le $\widehat{A}_{x}$-module $\widehat{B}_{y} / \mathfrak{p}$ est sans $\widehat{A}_{x}$-torsion, c'est donc un $\widehat{A}_{x}$-module de $\operatorname{dimension} \operatorname{dim}\left(\widehat{A}_{x}\right)$. La Proposition 16.1.9. de [31] montre alors que $\widehat{B}_{y} / \mathfrak{p}$ est un $\widehat{B}_{y}$-module de dimension $\operatorname{dim}\left(\widehat{A}_{x}\right)$. En particulier, $\widehat{B}_{y}$ est équidimensionnel et sans composante immergée, de dimension

$$
\begin{aligned}
\operatorname{dim}\left(\widehat{A}_{x}\right) & =\operatorname{dim}\left(\mathcal{W}_{\infty}\right) \\
& =q+n\left[F^{+}: \mathbb{Q}\right] \\
& =g+n^{2}|S|+\left[F^{+}: \mathbb{Q}\right] \frac{n(n-1)}{2}+n\left[F^{+}: \mathbb{Q}\right] \\
& =g+n^{2}|S|+\left[F^{+}: \mathbb{Q}\right] \frac{n(n+1)}{2}
\end{aligned}
$$

Par ailleurs, l'application $\omega_{X}$ de $U$ dans $V$ est surjective (voir la preuve de la Proposition 3.10). Comme $f$ est par ailleurs fini, l'image d'une composante irréductible de $X_{p}(\bar{\rho})$ par $\omega_{X}$ est une composante irréductible de $\mathcal{W}_{\infty}$. On en déduit que l'image d'une telle composante par $f$ est nécessairement de dimension supérieure ou égale à $\operatorname{dim}\left(\mathcal{W}_{\infty}\right)=$ $\operatorname{dim}\left(Z_{z}(\bar{\rho})\right)$, il s'agit donc d'une composante irréductible de $Z_{z}(\bar{\rho})$.

Corollaire 3.12. L'image d'une composante irréductible de $X_{p}(\bar{\rho})$ par le morphisme $\omega_{X}$ est un ouvert de Zariski de $\mathcal{W}_{\infty}$.

Démonstration. Le corollaire 3.11 montre qu'il suffit de prouver que l'image d'une composante irréductible de $Z_{z}(\bar{\rho})$ par $g$ est un ouvert Zariski de $\mathcal{W}_{\infty}$. C'est alors une conséquence de la preuve de [16, Cor. 6.4.4] puisque $Z_{z}(\bar{\rho})$ est une hypersurface de Fredholm par le Lemme 3.9, et que d'après [24, Thm. 4.2.2], une composante irréductible d'une hypersurface de Fredholm est une hypersurface de Fredholm.

3.4. Série principale localement $\mathbb{Q}_{p}$-analytique de $G_{p}$. Le groupe $G_{p}$ est le groupe des $\mathbb{Q}_{p}$-points du groupe $\mathbb{Q}_{p}$-algébrique $\prod_{v \in S_{p}} \operatorname{Res}_{F_{v}^{+} / \mathbb{Q}_{p}} \mathrm{GL}_{n, F_{v}^{+}}$, qui est un groupe réductif quasi-déployé. Le sous-groupe $B_{p}$ est le groupe des $\mathbb{Q}_{p}$-points du $\mathbb{Q}_{p}$-sous-groupe de Borel $\prod_{v \in S_{p}} \operatorname{Res}_{F_{v}^{+} / \mathbb{Q}_{p}} B_{F_{v}^{+}}$. Dans la suite, on appelle sous-groupe parabolique standard le groupe des $\mathbb{Q}_{p}$-points d'un sous-groupe parabolique de $\prod_{v \in S_{p}} \operatorname{Res}_{F_{v}^{+} / \mathbb{Q}_{p}} \mathrm{GL}_{n, F_{v}^{+}}$contenant $\prod_{v \in S_{p}} \operatorname{Res}_{F_{v}^{+} / \mathbb{Q}_{p}} B_{F_{v}^{+}}$(un tel groupe étant automatiquement défini sur $\mathbb{Q}_{p}$ ). Si $P$ est un sous-groupe parabolique standard, on note $\bar{P}$ le groupe des $\mathbb{Q}_{p}$-points du sous-groupe parabolique opposé. 
On note $\mathfrak{g}, \mathfrak{b}, \overline{\mathfrak{b}}, \mathfrak{p}, \overline{\mathfrak{p}}$ les $\mathbb{Q}_{p}$-algèbres de Lie des groupes $G_{p}, B_{p}, \bar{B}_{p}, P_{p}$ et $\bar{P}_{p}$, lorsque $P_{p}$ est un sous-groupe parabolique de $G_{p}$ contenant $B_{p}$ et $\bar{P}_{p}$ le parabolique opposé (relativement à $T_{p}$ ). Si $P_{p}$ est un tel sous-groupe parabolique, on note $L_{P}$ son sous-groupe de Levi contenant $T_{p}$. Si h est une $\mathbb{Q}_{p}$-algèbre de Lie, on note $\mathfrak{h}_{L}=\mathfrak{h} \otimes_{\mathbb{Q}_{p}} L$. Comme $L$ est choisie de sorte que $\operatorname{dim}_{L} \operatorname{Hom}\left(F^{+} \otimes_{\mathbb{Q}} \mathbb{Q}_{p}, L\right)=\left[F^{+}: \mathbb{Q}\right]$, on a une décomposition $\mathfrak{g}_{L} \simeq \bigoplus_{\tau \in \operatorname{Hom}\left(F^{+}, L\right)} \mathfrak{g}_{\tau}$, où $\mathfrak{g}_{\tau}=\mathfrak{g} \otimes_{F^{+}, \tau} L$. Le groupe de Weyl de $\mathfrak{g}_{L}$ se décompose alors canoniquement en un produit $\prod_{\tau \in \operatorname{Hom}\left(F^{+}, L\right)} W_{\tau}$, où $W_{\tau}$ est le groupe de Weyl de $\mathfrak{g}_{\tau}$.

Les résultats et preuves des $\$ 2$ et $\$ 3$ de [10] restent valables mutatis mutandis dans ce contexte. On peut définir la catégorie $\mathcal{O}_{\text {alg }}^{\overline{\mathfrak{p}}}$ de [37] comme dans [10, §2] et définir suivant Orlik et Strauch ([50]), pour toute représentation lisse de longueur finie $\pi_{P}$ de $L_{P}$, et tout objet $M$ de la catégorie $\mathcal{O}_{\text {alg }}^{\overline{\mathfrak{p}}}$, une représentation localement analytique admissible de $G_{p}$ notée $\mathcal{F}_{\bar{P}_{p}}^{G_{p}}\left(M, \pi_{P}\right)$.

De même, si $P$ désigne un sous-groupe parabolique standard de $\prod_{v \in S_{p}} \operatorname{Res}_{F_{v}^{+} / \mathbb{Q}_{p}} \mathrm{GL}_{n, F_{v}^{+}}$ et $M$ un objet de $\mathcal{O}_{\text {alg }}^{\bar{p}}$, on dit que $P$ est maximal pour $M$, si $M$ n'appartient pas à la sous-catégorie pleine $\mathcal{O}_{\text {alg }}^{\bar{q}}$ pour tout sous-groupe parabolique standard $Q$ contenant strictement $P$.

On a alors le résultat suivant.

Théorème 3.13 ([50], [10]). (i) La construction $\left(M, \pi_{P}\right) \mapsto \mathcal{F}_{\bar{P}_{p}}^{G_{p}}\left(M, \pi_{P}\right)$ est fonctorielle et exacte en les deux arguments.

(ii) Si $M$ est un $U\left(\mathfrak{g}_{L}\right)$-module simple, $P$ est maximal pour $M$ et $\pi_{P}$ est irréductible, la représentation $\mathcal{F}_{\bar{P}_{p}}^{G_{p}}\left(M, \pi_{P}\right)$ est irréductible.

Rappelons que $(-)^{\vee}$ désigne le foncteur de dualité sur la catégorie $\mathcal{O}_{\text {alg }}^{\overline{\mathfrak{b}}}$ (cf.[37, §3.2] et $[37, \S 9.3])$

Si $H \subset G_{p}$ est un sous-groupe et $\pi$ une représentation de $P_{p}$ sur un $L$-espace vectoriel, on note $\operatorname{Ind}_{H}^{G_{p}}(\pi)$ l'induite lisse non normalisée de $\pi$, c'est-à-dire le $L$-espace vectoriel des fonctions localement constantes $f: G_{p} \rightarrow \pi$ telles que $f(h g)=h \cdot f(g)$ pour tout $h \in H$ et $g \in G_{p}$. Il est muni de l'action lisse de $G_{p}$ par translation à droite. On note $\operatorname{ind}_{H}^{G_{p}}(\pi)$ le sous-espace stable par $G_{p}$ des fonctions à support compact.

3.5. Densité des points classiques. Rappelons que, pour $K$ extension finie de $\mathbb{Q}_{p}$, on a défini à la fin du $\$ 2.2$ un automorphisme $\iota_{K}$ de l'espace des caractères continus du groupe $T(K)$. On désigne par $\iota$ la version globale de cet automorphisme, c'est-à-dire que l'on pose $\iota=\left(\iota_{F_{v}^{+}}\right)_{v \in S_{p}}$, automorphisme de $\widehat{T}_{p, L}$.

Soit $x=(y, \delta) \in X_{p}(\bar{\rho})(L) \subset\left(\operatorname{Spf}\left(R_{\infty}\right)^{\text {rig }} \times \widehat{T}_{p, L}\right)(L)$. Notons $\mathfrak{p}_{y}$ l'idéal maximal de $R_{\infty}\left[\frac{1}{p}\right]$ correspondant au point $y \in \operatorname{Spf}\left(R_{\infty}\right)^{\mathrm{rig}}(L)=\operatorname{Hom}\left(R_{\infty}\left[\frac{1}{p}\right], L\right)$ et posons, pour le moment, $\Pi=\Pi_{\infty}\left[\mathfrak{p}_{y}\right]$. De la caractérisation des points de $X_{p}(\bar{\rho})$ donnée par la Proposition 3.7, on déduit que

$$
\left.\operatorname{Hom}_{T_{p}}\left(\delta, J_{B_{p}}\left(\Pi^{\mathrm{an}}\right)\right)\right) \neq 0 .
$$

Supposons que $\delta$ soit un caractère localement algébrique de $T_{p}$ de poids dominant $\lambda \in$ $\prod_{v \in S_{p}}\left(\mathbb{Z}^{n}\right)^{\left[F_{v}^{+}: \mathbb{Q}_{p}\right]}$. Par définition, la représentation $\Pi^{\text {an }}$ est très fortement admissible (cf. [28, Def. 0.12]), on peut donc appliquer le Théorème 4.3 de [11] qui donne un isomorphisme

$$
\operatorname{Hom}_{G_{p}}\left(\mathcal{F}_{\bar{B}_{p}}^{G_{p}}\left(\left(U\left(\mathfrak{g}_{L}\right) \otimes_{U\left(\overline{\mathfrak{b}}_{L}\right)}(-\lambda)\right)^{\vee}, \delta_{B_{p}}^{-1} \delta \delta_{\lambda}^{-1}\right), \Pi^{\mathrm{an}}\right) \simeq \operatorname{Hom}_{T_{p}}\left(\delta, J_{B_{p}}\left(\Pi^{\mathrm{an}}\right)\right) .
$$


L'apparition du caractère module $\delta_{B_{P}}^{-1}$ par rapport à loc. cit. vient du fait que nous avons choisi la paramétrisation d'Emerton pour le foncteur $J_{B_{p}}$.

Notons $L(\lambda)$ le $U\left(\mathfrak{g}_{L}\right)$-module simple de plus haut poids $\lambda$ relativement à $\mathfrak{b}_{L}$. Comme $\lambda$ est dominant, il s'agit d'un $L$-espace vectoriel de dimension finie. De plus, l'action de $\mathfrak{g}$ sur $L(\lambda)$ s'étend de façon unique en une représentation algébrique du groupe $G_{p}$. Par ailleurs, il existe une injection de $U\left(\mathfrak{g}_{L}\right)$-modules $L(\lambda)^{\prime} \hookrightarrow\left(U\left(\mathfrak{g}_{L}\right) \otimes_{U\left(\overline{\mathfrak{b}}_{L}\right)}(-\lambda)\right)^{\vee}$. Par le (i) du Théorème 3.13, cette injection induit une surjection continue entre représentations localement analytiques admissibles de $G_{p}$

$$
\mathcal{F}_{\bar{B}_{p}}^{G_{p}}\left(\left(U\left(\mathfrak{g}_{L}\right) \otimes_{U\left(\overline{\mathfrak{b}}_{L}\right)}(-\lambda)\right)^{\vee}, \delta_{B_{p}}^{-1} \delta \delta_{\lambda}^{-1}\right) \rightarrow \mathcal{F}_{G_{p}}^{G_{p}}\left(L(\lambda)^{\prime}, \operatorname{Ind}_{\bar{B}_{p}}^{G_{p}}\left(\delta_{B_{p}}^{-1} \delta \delta_{\lambda}^{-1}\right)\right) .
$$

Lorsque la représentation $\operatorname{Ind} \frac{G_{p}}{\bar{B}_{p}}\left(\delta_{B_{p}}^{-1} \delta \delta_{\lambda}^{-1}\right)$ est irréductible, elle est isomorphe à $\operatorname{Ind}_{B_{p}}^{G_{p}}\left(\delta \delta_{\lambda}^{-1}\right)$ en utilisant les isomorphismes

$$
\operatorname{Ind}_{\bar{B}_{p}}^{G_{p}}(\chi) \simeq \operatorname{Ind}_{B_{p}}^{G_{p}}\left(\chi^{w_{0}}\right) \text { et } \operatorname{Ind}_{B_{p}}^{G_{p}}(\chi) \simeq \operatorname{Ind}_{B_{p}}^{G_{p}}\left(\chi^{w_{0}} \delta_{B_{p}}\right)
$$

entre induites paraboliques lisses, $w_{0}$ désignant l'élément du groupe de Weyl de $G_{p}$ de longueur maximale (relativement à l'ensemble de racines simples défini par le choix de $\left.B_{p}\right)$.

Au final on obtient une injection

$$
\operatorname{Hom}_{G_{p}}\left(L(\lambda) \otimes_{L} \operatorname{Ind}_{\bar{B}_{p}}^{G_{p}}\left(\delta_{B_{p}}^{-1} \delta \delta_{\lambda}^{-1}\right), \Pi\right) \hookrightarrow \operatorname{Hom}_{T_{p}}\left(\delta, J_{B_{p}}\left(\Pi^{\mathrm{an}}\right)\right) .
$$

Définition 3.14. Un point $x=(y, \delta) \in\left(\operatorname{Spf}\left(R_{\infty}\right)^{\text {rig }} \times \widehat{T}_{p, L}\right)(L)$ est dit classique de poids $\lambda$ si le membre de gauche de (3.8) est non nul.

Si $x=(y, \delta) \in\left(\operatorname{Spf}\left(R_{\infty}\right)^{\text {rig }} \times \widehat{T}_{p, L}\right)(L)$, et $v \in S_{p}$, on note $y_{v}$ la composante en $v$ de l'image de $y$ dans $\mathfrak{X}^{\square}\left(\bar{\rho}_{p}\right) \simeq \prod_{v \in S_{p}} \mathfrak{X}^{\square}\left(\bar{\rho}_{\tilde{v}}\right)$, on note alors $r_{y, v}$ la classe d'isomorphisme de représentation de $\mathcal{G}_{F_{\tilde{v}}}$ correspondant à $y_{v}$. De même on note $\delta_{v}$ la composante en $v$ de $\delta$ via la décomposition $\widehat{T}_{p} \simeq \prod_{v \in S_{p}} \widehat{T}_{v}$.

Proposition 3.15. Soit $(y, \iota(\delta)) \in X_{p}(\bar{\rho})(L)$ un point classique de poids $\lambda$. Supposons que pour tout $v \in S_{p}$, la représentation $\operatorname{Ind}_{B_{v}}^{G_{v}}\left(\delta_{v} \delta_{\lambda_{v}}^{-1}\right)$ soit absolument irréductible. Alors la représentation $r_{y, v}$ est cristalline de poids de Hodge-Tate

$$
\left(k_{\tau, i}=\lambda_{\tau, i}-(i-1)\right)_{\tau \in \operatorname{Hom}\left(F^{+}, L\right), 1 \leq i \leq n} .
$$

De plus, l'ensemble des valeurs propres du Frobenius cristallin de $r_{y, v}$, est

$$
\left\{\varphi_{v, i}=\delta_{v, i}\left(\varpi_{v}\right) \cdot \prod_{\tau \in \operatorname{Hom}\left(F_{v}^{+}, L\right)} \delta_{k_{\tau, i}}\left(\varpi_{v}\right)^{-1} \mid 1 \leq i \leq n\right\} .
$$

Si de plus le raffinement $\left(\varphi_{v, i}\right)_{1 \leq i \leq n}$ est non critique, alors $\left(y_{v}, \delta_{v}\right) \in X_{\text {tri }}^{\square}\left(\bar{\rho}_{\tilde{v}}\right)$.

Démonstration. Si $x=(y, \iota(\delta))$ est un point classique de poids dominant $\lambda$, le caractère $\chi=\iota(\delta) \delta_{\lambda}^{-1}$ est lisse et non ramifié. De plus, $\chi$ se décompose naturellement en un produit tensoriel $\bigotimes_{v \in S_{p}} \chi_{v}$, où $\chi_{v}$ est un caractère lisse et non ramifié de $T_{v}$. Comme la représentation $\operatorname{Ind}_{B_{v}}^{G_{v}}\left(\chi_{v}\right)$ est absolument irréductible, on a $\chi_{v, i} \chi_{v, j}^{-1} \notin\left\{|\cdot|_{F_{v}}^{j-i+1}\right\}$ pour $i \neq j$ (voir par exemple [44]). Soit $\mathcal{H}(\lambda)$ l'algèbre de Hecke $\operatorname{End}_{G_{p}}\left(\operatorname{ind}_{K_{p}}^{G_{p}} L(\lambda)\right)$. Il existe un unique caractère $\theta_{\chi}: \mathcal{H}(\lambda) \rightarrow L$ tel que

$$
L(\lambda) \otimes_{L} \operatorname{Ind}_{B_{p}}^{G_{p}}(\chi) \simeq \operatorname{ind}_{K_{p}}^{G_{p}}(L(\lambda)) \otimes_{\mathcal{H}(\lambda)} L\left(\theta_{\chi}\right) .
$$


L'existence d'un tel caractère résulte par exemple de l'isomorphisme du bas de la page 670 de [56] et de la remarque suivant la Conjecture page 673 de loc. cit..

Par réciprocité de Frobenius, on a un isomorphisme

$$
\operatorname{Hom}_{K_{p}}\left(L(\lambda), \Pi_{\infty}\right) \simeq \operatorname{Hom}_{G_{p}}\left(\operatorname{ind}_{K_{p}}^{G_{p}} L(\lambda), \Pi_{\infty}\right),
$$

qui fournit ainsi une action de l'algèbre commutative $\mathcal{H}(\lambda)$ sur l'espace $\operatorname{Hom}_{K_{p}}\left(L(\lambda), \Pi_{\infty}\right)$, que l'on note $\Pi_{\infty}(\lambda)$. Comme le point $x$ est classique, il existe un morphisme non nul de $L(\lambda) \otimes_{L} \operatorname{Ind}_{P_{p}}^{G_{p}}(\chi)$ dans $\Pi_{\infty}\left[\mathfrak{p}_{y}\right]$, l'isomorphisme (3.9) implique alors qu'il existe un sousespace non nul de $\Pi_{\infty}(\lambda)\left[\mathfrak{p}_{y}\right]$ sur lequel $\mathcal{H}(\lambda)$ agit via $\theta_{\chi}$. Comme le point $x$ est classique de poids $\lambda$, on a $\Pi_{\infty}(\lambda)\left[\mathfrak{p}_{y}\right] \neq 0$. Le Lemme 4.16 de [15], appliqué à a représentation $\sigma=L(\lambda)$, implique alors que pour $v \in S_{p}$, la représentation $r_{y, v}$ de $\mathcal{G}_{F_{\tilde{v}}}$ est cristalline de poids $\mathbf{k}_{v}=\left(k_{\tau, i}\right)_{\tau \in \operatorname{Hom}\left(F_{v}^{+}, L\right), 1 \leq i \leq n}$ où $k_{\tau, i}=\lambda_{\tau, i}-(i-1)$ et que le Frobenius cristallin linéarisé de $r_{y, v}$ a pour valeurs propres $\left(\varphi_{i, v}\right)$ où $\varphi_{i, v}=\chi_{i}\left(\varpi_{v}\right)\left|\varpi_{v}\right|_{v}^{i-n}$. Plus précisément les décompositions $G_{p}=\prod_{v \in S_{p}} G_{v}$ et $K_{p}=\prod_{v \in S_{p}} K_{v}$ permettent de décomposer $\mathcal{H}(\lambda)$ en produit tensoriel $\bigotimes_{v \in S_{p}} \mathcal{H}\left(\lambda_{v}\right)$ où $\mathcal{H}\left(\lambda_{v}\right)$ désigne l'algèbre des endomorphismes de $\operatorname{ind}_{K_{v}}^{G_{v}} L\left(\lambda_{v}\right)$. Le Théorème 4.1 de [15] permet alors de construire un unique morphisme de $L$-algèbres $\eta_{v}: \mathcal{H}\left(\lambda_{v}\right) \rightarrow R_{\bar{\rho}_{\tilde{v}}}^{\square, k_{v}-\text { cr }}\left[\frac{1}{p}\right]$ qui interpole la correspondance de Langlands comme normalisée dans le $\$ 2.2$. On peut alors, en raisonnant exactement comme dans la preuve de [15, Lem. 4.16], prouver que l'action de $R_{\bar{\rho}_{\tilde{v}}}^{\square} \operatorname{sur} \Pi_{\infty}(\lambda)$ se factorise par $R_{\bar{\rho}_{\tilde{v}}}^{\square, k_{v} \text {-cr }}$ et que l'action de $\mathcal{H}\left(\lambda_{v}\right)$ se déduit alors de cette action de $R_{\bar{p}_{\bar{v}}}^{\square, k_{v}-\mathrm{cr}}\left[\frac{1}{p}\right]$ via $\eta_{v}$.

Tout ceci implique au final que $r_{y, v}$ est trianguline et a un paramètre égal à

$$
\delta_{\lambda_{v}} \chi_{v} \cdot\left(1, \varepsilon^{-1} \circ \operatorname{rec}_{F_{v}^{+}}, \ldots, \varepsilon^{1-n} \circ \operatorname{rec}_{F_{v}^{+}}\right) \cdot \delta_{B_{v}}^{-1}=\delta_{v} .
$$

La dernière assertion est immédiate car, si le raffinement est non critique, le caractère $\delta_{v}$ est un paramètre de $r_{y, v}$ d'après [4, Prop. 2.4.7].

Il est commode d'introduire la notion suivante.

Définition 3.16. Un point $x=(y, \delta) \in X_{p}(\bar{\rho})(L) \subset\left(\operatorname{Spf}\left(R_{\infty}\right)^{\mathrm{rig}} \times \widehat{T}_{p, L}\right)(L)$ est dit très classique de poids $\lambda$ si la restriction de $\delta$ à $T_{p}^{0}$ est un caractère algébrique de poids entier dominant $\lambda$ et si

$$
L(\lambda) \otimes_{L} \operatorname{Ind} \frac{G_{p}}{G_{p}}\left(\delta \delta_{B_{p}}^{-1} \delta_{\lambda}^{-1}\right)
$$

est le seul sous-quotient irréductible de $\operatorname{Ind}_{\bar{B}_{p}}^{G_{p}}\left(\delta \delta_{B_{p}}^{-1}\right)^{\text {an }}$ sur lequel existe une norme $G_{p^{-}}$ invariante.

Remarque 3.17. La représentation $\operatorname{Ind}_{\bar{B}_{p}}^{G_{p}}\left(\delta \delta_{B_{p}}^{-1}\right)^{\text {an }}$ a les mêmes sous-quotients irréductibles que

$$
\mathcal{F}_{\bar{B}_{p}}^{G_{p}}\left(\left(U\left(\mathfrak{g}_{L}\right) \otimes_{U\left(\overline{\mathfrak{b}}_{p}\right)_{L}}(-\lambda)\right)^{\vee}, \delta \delta_{\lambda}^{-1} \delta_{B_{p}}^{-1}\right) .
$$

Il résulte de cette remarque et de (3.7) qu'un point très classique est en particulier classique. L'assertion inverse étant fausse!

Théorème 3.18. Les points classiques forment une partie Zariski-dense et d'accumulation de $X_{p}(\bar{\rho})$. Plus précisément, pour tout point $x$ de $X_{p}(\bar{\rho})$ de poids algébrique et $X$ une composante irréductible de $X_{p}(\bar{\rho})$ contenant $x$, il existe un voisinage affinoïde $U$ de $x$ dans $X$ et une partie $W$ de $\widehat{T}_{p, L}^{0}$ tels que $\omega_{X}^{-1}\left(\operatorname{Spf}\left(S_{\infty}\right)^{\mathrm{rig}} \times W\right) \cap U$ soit Zariski-dense dans $U$ et constitué de points très classiques, à paramètre régulier non critique. 
Démonstration. Soit $x \in X_{p}(\bar{\rho})$ de poids algébrique. La Proposition 3.10 nous permet de considérer $U$ un voisinage affinoïde et connexe de $x$ dans la composante $X$ fixée, tel que $\omega_{X}(U)$ est un ouvert affinoïde et la restriction de $\omega_{X}$ à $U$ est une application finie de $U$ sur $\omega_{X}(U)$. Comme $U$ est affinoïde, les $n\left|S_{p}\right|$ fonctions analytiques rigides $(y, \delta) \mapsto\left(\delta \delta_{B_{p}}^{-1}\right)\left(\gamma_{\tilde{v}, i}\right)$ sont bornées sur $U$ (rappelons que l'on a défini $\gamma_{\tilde{v}, i}$ comme l'image d'une uniformisante $\varpi_{v}$ par le cocaractère $\beta_{i}$ de $\mathbb{G}_{m, F_{v}^{+}}$dans $\left.\mathrm{GL}_{n, F_{\tilde{v}}} \simeq G_{F_{v}^{+}}\right)$. Fixons donc $C>0$ tel que

$$
-C \leq v_{F_{v}^{+}}\left(\left(\delta_{v} \delta_{B_{v}}^{-1}\right)\left(\gamma_{\tilde{v}, i}\right)\right) \leq C
$$

pour tout $v \in S_{p}, 1 \leq i \leq n$ et $(y, \delta) \in U$. Soit $Z$ l'ensemble des caractères algébriques dominants $\delta \in \widehat{T}_{p, L}^{0}$ de poids $\lambda$ tels que pour tout $\tau: F^{+} \hookrightarrow \overline{\mathbb{Q}}_{p}$,

$$
\begin{aligned}
& \lambda_{1, \tau}-\lambda_{2, \tau}>2(C+1), \\
& \lambda_{i, \tau}-\lambda_{i+1, \tau}>\lambda_{i-1, \tau}-\lambda_{i, \tau}+(C+1), \text { pour } i \geq 2 .
\end{aligned}
$$

Nous allons prouver que tous les points de $\omega_{X}^{-1}\left(\operatorname{Spf}\left(S_{\infty}\right)^{\text {rig }} \times Z\right)$ sont classiques et non critiques.

En effet, l'ensemble $Z$ s'accumule en tout caractère algébrique de $\widehat{T}_{p, L}^{0}$, on en conclut que si $V$ est un ouvert irréductible de $\widehat{T}_{p, L}^{0}$ contenant un poids algébrique, alors $V \cap Z$ est Zariski-dense dans $V$, ce qui implique que $\omega_{X}(U) \cap\left(\operatorname{Spf}\left(S_{\infty}\right)^{\text {rig }} \times Z\right)$ est Zariski-dense dans $\omega_{X}(U)$. Par finitude et surjectivité de $\left.\omega_{X}\right|_{U}$, on en déduit que $\omega_{X}^{-1}\left(\operatorname{Spf}\left(S_{\infty}\right)^{\text {rig }} \times Z\right) \cap U$ est Zariski-dense dans l'ouvert irréductible $U$. En effet, si ce n'était pas le cas, par connexité de $U$, le plus petit fermé analytique contenant $\omega_{X}^{-1}\left(\operatorname{Spf}\left(S_{\infty}\right)^{\text {rig }} \times Z\right) \cap U$ serait de dimension $<\operatorname{dim}(U)$, donc son image par $\omega_{X}$ également. Or, par finitude de $\omega_{X}: U \rightarrow \omega_{X}(U)$, cette image est un fermé analytique contenant $\left(\operatorname{Spf}\left(S_{\infty}\right)^{\text {rig }} \times Z\right) \cap \omega_{X}(U)$, ce qui contredit la Zariski-densité de cet ensemble dans $\omega_{X}(U)$ et le fait que $\operatorname{dim}(U)=\operatorname{dim}\left(\omega_{X}(U)\right)$.

Soit donc $x \in \omega_{X}^{-1}\left(\operatorname{Spf}\left(S_{\infty}\right)^{\text {rig }} \times Z\right) \cap U$. Reprenons les notations de la discussion suivant la Définition 3.14 et de la preuve de la Proposition 3.15 .

Nous affirmons que pour tout sous-groupe de Levi standard $L_{I}$ de $\mathrm{GL}_{n}$, et pour tout $v \in S_{p}$, l'induite parabolique lisse $\operatorname{Ind}_{\bar{B}_{v} \cap L_{I, v}}^{L_{I, v}}\left(\chi_{v} \delta_{B_{v}}^{-1}\right)$ est irréductible. En effet, d'après [61, Thm. 4.2], il suffit pour cela de vérifier que pour tout $i<j$ on a

$$
v_{F_{v}^{+}}\left(\chi_{v, j}\left(\varpi_{v}\right) \chi_{v, i}\left(\varpi_{v}\right)^{-1}\left|\varpi_{v}\right|_{v}^{j-i}\right)>1
$$

c'est-à-dire

$$
v_{F_{v}^{+}}\left(\delta_{v, j}\left(\varpi_{v}\right) \delta_{v, i}\left(\varpi_{v}\right)^{-1}\left|\varpi_{v}\right|_{v}^{j-i}\right)>1+\sum_{\tau \in \operatorname{Hom}\left(F_{v}^{+}, L\right)}\left(\lambda_{\tau, j}-\lambda_{\tau, i}\right),
$$

ce qui est une conséquence de la condition (3.11). Ainsi, pour tout sous-groupe parabolique standard $P$ de $G_{p}$, si $L_{P}$ désigne l'unique sous-groupe de Levi de $P$ contenant $T_{p}$, la représentation $\operatorname{Ind}_{L_{P} \cap \bar{B}_{p}}^{L_{P}}\left(\chi \delta_{B_{p}}^{-1}\right)$, isomorphe à $\bigotimes_{v \in S_{p}} \operatorname{Ind}_{L_{P_{v}} \cap \bar{B}_{v}}^{\mathrm{GL}_{n}\left(F_{+}^{+}\right)}\left(\chi_{v} \delta_{B_{v}}^{-1}\right)$, est irréductible.

Pour prouver que $x$ est très classique, il suffit de prouver que $L(\lambda) \otimes_{L} \operatorname{Ind}_{B_{p}}^{G_{p}}(\chi)$ est le seul sous-quotient irréductible de $\operatorname{Ind} \frac{G_{p}}{\bar{B}_{p}}\left(\delta \delta_{B_{p}}^{-1}\right)$ an admettant une norme invariante.

Il résulte du Théorème 3.13 que les sous-quotients irréductibles de la série principale localement analytique $\operatorname{Ind} \frac{G_{p}}{\bar{B}_{p}}\left(\delta \delta_{B_{p}}^{-1}\right)^{\text {an }}$ sont exactement les $\mathcal{F}_{\bar{P}}^{G_{p}}(M, \pi)$, où $M$ est un sousquotient irréductible de $U\left(\mathfrak{g}_{L}\right) \otimes_{U\left(\overline{\mathfrak{b}}_{L}\right)}(-\lambda), \bar{P}$ le sous-groupe parabolique maximal pour $M$ et $\pi$ la représentation lisse irréductible $\operatorname{Ind} \frac{L_{P}}{\bar{B}_{p} \cap L_{P}}\left(\chi \delta_{\bar{B}_{p}}^{-1}\right)$. Si $M$ est un tel sous-quotient, alors il existe $w$ dans le groupe de Weyl $W$ de $\mathfrak{g}_{L}$ tel que $M$ est isomorphe à $M_{-w \cdot \lambda}$, le 
$U\left(\mathfrak{g}_{L}\right)$-module simple de plus haut poids $-w \cdot \lambda$ (relativement au sous-groupe de Borel $\left.\overline{\mathfrak{b}}_{L}\right)$. Nous allons prouver que si $\mathcal{F}_{\bar{P}}^{G_{p}}\left(M_{-w \cdot \lambda}, \pi\right)$ est muni d'une norme stable par $G_{p}$, alors $w=1$. Si $P$ est un sous-groupe parabolique de $G_{p}$ contenant $B_{p}$, notons $L_{P}$ le facteur de Levi de $P$ qui contient $T$ et $N_{P}$ le radical unipotent de $P$. De plus on note $Z_{P}$ le centralisateur de $L_{P}$ dans $G_{p}$. On note $Z_{P}^{+}$, comme en $[10,(12)]$, le sous-monoïde de $Z_{P}$ constitué des $g \in Z_{P}$ tels que $g\left(N_{P} \cap K_{p}\right) g^{-1} \subset N_{P} \cap K_{p}$. Alors, d'après le corollaire 3.5 de [10], si $\mathcal{F}_{\bar{P}_{p}}^{G_{p}}\left(M_{-w \cdot \lambda}, \pi_{P}\right)$ admet une norme $G_{p}$-invariante, on doit avoir

$$
\delta_{w \cdot \lambda}(z) \chi(z) \delta_{B_{p}}^{-1}(z) \in \mathcal{O}_{L}
$$

pour tout $z \in Z_{P}^{+}$. Décomposons $P_{p}$ en un produit $P=\prod_{v \in S_{p}} P_{v}$ de sous-groupes paraboliques selon la décomposition $G_{p}=\prod_{v \in S_{p}} \mathrm{GL}_{n}\left(F_{v}^{+}\right)$. Pour tout $v \in S_{p}$, le groupe $P_{v}$ est le groupe des $F_{v}^{+}$-points d'un sous-groupe parabolique standard $P_{I_{v}}$ de $\mathrm{GL}_{n}$, pour $I_{v} \subset \Delta$. Si, pour tout $v \in S_{p}$, on choisit $1 \leq i_{v} \leq n-1$ tel que $i_{v} \notin I_{v}$, et que l'on pose $\gamma_{v, i_{v}}=\beta_{i_{v}}\left(\varpi_{v}\right)$, l'élément $\left(\gamma_{v, i_{v}}\right)_{v \in S_{p}}$ de $G_{p}$ est en fait dans $Z_{P}^{+}$.

D'après [37, pages 186-187], pour $v \in S_{p}$, l'ensemble $I_{v}$ est la plus grande partie de $\Delta$ telle que $w_{\tau} \cdot \lambda_{\tau}$ est un poids dominant du groupe algébrique $L_{P_{v}} \times_{F_{v}} L$ (relativement au Borel $\left.\left(B_{v} \cap L_{P_{v}}\right) \times_{F_{v}^{+}} L\right)$ pour $\tau \in \operatorname{Hom}\left(F_{v}^{+}, L\right)$. Si $w_{v} \neq 1$, on a $I_{v} \neq\{1, \ldots, n-1\}$ et, d'après la proposition 5.4 appliquée à $\lambda+\rho$, il est possible de trouver une racine simple $\alpha_{v, i_{v}} \notin I_{v}$ telle que

$$
\left\langle w \cdot \lambda-\lambda, \beta_{v, i_{v}}\right\rangle \leq-\min _{\tau, i}\left(\lambda_{\tau, i}-\lambda_{\tau, i+1}\right)
$$

Mais alors, puisque

$$
\delta_{\lambda}\left(\gamma_{v, i_{v}}\right)=\varpi_{v}\left\langle\sum_{\tau \in \operatorname{Hom}\left(F_{v}^{+}, L\right)} \lambda_{\tau}, \beta_{i_{v}}\right\rangle,
$$

on a avec (3.10) et (3.11) :

$$
\begin{aligned}
\left|\delta_{w \cdot \lambda}\left(\gamma_{v, i_{v}}\right) \chi\left(\gamma_{v, i_{v}}\right) \delta_{B_{v}}\left(\gamma_{v, i_{v}}\right)^{-1}\right|_{L} & =\left|\delta_{w \cdot \lambda-\lambda}\left(\gamma_{v, i_{v}}\right) \delta_{v}\left(\gamma_{v, i_{v}}\right) \delta_{B_{v}}\left(\gamma_{v, i_{v}}\right)^{-1}\right|_{L} \\
& \geq\left|\varpi_{v}\right|_{L}^{C-\min _{i, \tau}\left(\lambda_{\tau, i}-\lambda_{\tau, i+1}\right)}>1
\end{aligned}
$$

Ainsi, en posant $z=\left(\gamma_{v, i_{v}}\right)_{v \in S_{p}}$, on a bien $z \in Z_{P}^{+}$, tel que

$$
\left|\delta_{w \cdot \lambda-\lambda}(z) \chi(z) \delta_{B_{p}}^{-1}(z)\right|_{L}>1
$$

ce qui implique par (3.12) que $\mathcal{F}_{\bar{P}_{p}}^{G_{p}}\left(M_{-w \cdot \lambda}, \pi_{P}\right)$ ne peut avoir de norme $G_{p}$-invariante à moins que $w=1$. Ceci prouve que le point $x$ est très classique. Par ailleurs, les inégalités (3.11) impliquent également que le raffinement de $r_{y, v}$ déduit de $\delta_{v}$ est non critique (on peut par exemple comparer ces inégalités aux conditions de [35, Lem. 2.9]). Enfin, le paramètre $\iota^{-1}(\delta)$ est régulier, ou de façon équivalente on a $\chi_{i, v} \neq \chi_{j, v}|\cdot|{ }_{v}^{j-i \pm 1}$ pour tous $i \neq j$ et $v \in S_{p}$, ce qui est une conséquence du fait que les représentations $\operatorname{Ind}_{L_{P} \cap \bar{B}_{p}}^{G_{p}}(\chi)$ sont toutes irréductibles.

Nous avons prouvé que l'ensemble des points classiques non critiques est un ensemble d'accumulation dans $X_{p}(\bar{\rho})$. Pour conclure qu'il est dense au sens de Zariski dans $X_{p}(\bar{\rho})$, il suffit de montrer que toute composante irréductible de $X_{p}(\bar{\rho})$ contient un point de poids algébrique. Or c'est une conséquence du Corollaire 3.12 puisque les poids algébriques forment une partie dense au sens de Zariski dans $\mathcal{W}_{\infty}$.

Corollaire 3.19. L'espace analytique $X_{p}(\bar{\rho})$ est réduit. 
Démonstration. Il suffit de prouver que chaque composante irréductible de $X_{p}(\bar{\rho})$ contient un point $x$ dont l'anneau local est réduit. En effet, soit $\mathcal{I}$ le nilradical de $X_{p}(\bar{\rho})$ (cf. $[9$, $\S 9.5 .1])$. Il s'agit d'un faisceau cohérent d'idéaux sur $X_{p}(\bar{\rho})$. Comme d'après le Corollaire 3.11, l'espace $X_{p}(\bar{\rho})$ est sans composante immergée, le support du faisceau $\mathcal{I}$ est une union de composantes irréductibles. Si chaque composante irréductible contient un point réduit, c'est-à-dire un point dont l'anneau local complété est réduit, alors $\mathcal{I}=0$ et $X_{p}(\bar{\rho})$ est réduit. D'après le Théorème 3.18 , toute composante irréductible de $X_{p}(\bar{\rho})$ contient un point très classique, il suffit donc de prouver que $X_{p}(\bar{\rho})$ est réduit en tout point très classique.

On raisonne alors comme dans [17, Prop. 3.9], l'espace des poids étant ici remplacé par $\mathcal{W}_{\infty}$. Soient $x \in X_{p}(\bar{\rho})$ un point très classique, disons de poids $\lambda$, et $z$ son image dans $\mathcal{W}_{\infty}$

Soit $U=\operatorname{Sp}(B)$ un voisinage affinoïde de $x$ comme dans la Proposition 3.10, et $V=$ $\operatorname{Sp}(A)$ un ouvert affinoïde de $\mathcal{W}_{\infty}$ tel que $\omega_{X}$ induise une application finie surjective de $U$ sur $V$ et que $U$ soit une composante connexe de $\omega_{X}^{-1}(V)$. On note $M=\Gamma\left(U, \mathcal{M}_{\infty}\right)$. Il s'agit d'un $A$-module projectif de type fini et l'action de $B$ sur $M$ identifie $B$ à une sous- $A$-algèbre de $\operatorname{End}_{A}(M)$.

On déduit du Théorème 3.18, quitte à rétrécir $U$, l'existence d'un ensemble $Z$ de poids algébriques dominants de $\widehat{T}_{p, L}^{0}$ tels que $\left(\operatorname{Spf}\left(S_{\infty}\right)^{\text {rig }} \times Z\right) \cap V$ soit dense au sens de Zariski dans $V$ et que tous les points de $\omega_{X}^{-1}\left(\operatorname{Spf}\left(S_{\infty}\right)^{\text {rig }} \times Z\right) \cap U$ soient très classiques et de paramètre régulier. On peut également, quitte à rétrécir $U$ et $V$, supposer que $V$ est isomorphe à un produit $V_{1} \times V_{2}$, où $V_{1}$ et $V_{2}$ sont des ouverts affinoïdes connexes de $\operatorname{Spf}\left(S_{\infty}\right)^{\text {rig }}$ et de $\widehat{T}_{p, L}^{0}$. Nous allons prouver que pour tout $\lambda \in Z$, il existe un ouvert de Zariski $V_{\lambda}$, dense au sens de Zariski, de $V \cap\left(\operatorname{Spf}\left(S_{\infty}\right)^{\text {rig }} \times\left\{\delta_{\lambda}\right\}\right)=V_{1} \times\left\{\delta_{\lambda}\right\}$ tel que si $z \in V_{\lambda}$, le $B$-module $M \otimes_{A} k(z)$ est semi-simple. Comme $\bigcup_{\lambda \in Z} V_{\lambda}$ est alors une partie dense au sens de Zariski de $V$ (ce qui se déduit de la forme de $V$ ), on conclut comme dans la preuve de la Proposition 4.9 de [17].

Prouvons donc l'existence d'un tel $V_{\lambda}$. Comme $B$ est la sous- $A$-algèbre de $\operatorname{End}_{A}(M)$ engendrée par $A$, l'image de $R_{\infty}$ et la sous-algèbre engendrée par $T_{p}^{+}$, il suffit de construire $V_{\lambda}$ tel que pour $z \in V_{\lambda}$, le $k(z)$-espace vectoriel $M \otimes_{A} k(z)$ est semi-simple à la fois comme $R_{\infty}$-module et comme $k(z)\left[T_{p}^{+}\right]$-module.

Si $z \in \operatorname{Spf}\left(S_{\infty}\right)^{\text {rig }} \times\left\{\delta_{\lambda}\right\}$, notons $\mathfrak{p}_{z} \subset S_{\infty}\left[\frac{1}{p}\right]$ l'idéal maximal correspondant et $k(z)$ son corps résiduel. Notons $\Sigma=\operatorname{Hom}_{k(z)}\left(M \otimes_{A} k(z), k(z)\right)$. Comme $M \otimes_{A} k(z)$ est l'ensemble des sections de $\mathcal{M}_{\infty}$ sur l'ensemble fini $U \cap \omega_{X}^{-1}(z)$, la représentation $\Sigma$ est isomorphe à un facteur direct de $J_{B_{p}}\left(\Pi_{\infty}^{R}-\text { an }\left[\mathfrak{p}_{z}\right]\right)^{T_{p}^{0}=\delta_{\lambda}}$. On peut donc écrire $\Sigma \simeq \delta_{\lambda} \otimes_{L} \Sigma_{\infty}$, où $\Sigma_{\infty}$ est une représentation lisse non ramifiée de dimension finie de $T_{p}$.

Le Théorème 4.3 de [11] permet alors d'associer à tout morphisme $\Sigma \rightarrow J_{B_{p}}\left(\Pi_{\infty}^{R_{\infty}-\text { an }}\left[\mathfrak{p}_{z}\right]\right)$ un morphisme de représentations $G_{p}$-analytiques

$$
\mathcal{F}_{\bar{B}_{p}}^{G_{p}}\left(\left(U\left(\mathfrak{g}_{L}\right) \otimes_{U\left(\overline{\mathfrak{b}}_{L}\right)}(-\lambda)\right)^{\vee}, \Sigma_{\infty}\left(\delta_{B_{p}}^{-1}\right)\right) \rightarrow \Pi_{\infty}\left[\mathfrak{p}_{z}\right]
$$

Comme deux caractères lisses de $T_{p}$ ont une extension lisse non triviale si et seulement si ils sont égaux, on en conclut que les facteurs de Jordan-Hölder de $\Sigma_{\infty}$ coïncident avec les constituants du socle de $\Sigma_{\infty}$. Remarquons de plus qu'une extension lisse entre deux caractères non ramifiés de $T_{p}$ est non ramifiée, donc $\Sigma_{\infty}$ est une représentation de dimension finie du groupe $T_{p} / T_{p}^{0}$. Or par construction de $\lambda, U$ et $V$, si $\chi$ est un caractère 
lisse du socle de $\Sigma_{\infty}$, le seul sous-quotient de

$$
\mathcal{F}_{\bar{B}_{p}}^{G_{p}}\left(\left(U\left(\mathfrak{g}_{L}\right) \otimes_{U\left(\overline{\mathfrak{b}}_{L}\right)}(-\lambda)\right)^{\vee}, \chi \delta_{B_{p}}^{-1}\right)
$$

admettant une norme $G_{p}$-invariante est son quotient localement algébrique. On en déduit donc que les seuls sous-quotients de

$$
\mathcal{F}_{\bar{B}_{p}}^{G_{p}}\left(\left(U\left(\mathfrak{g}_{L}\right) \otimes_{U\left(\overline{\mathfrak{b}}_{L}\right)}(-\lambda)\right)^{\vee}, \Sigma_{\infty}\left(\delta_{B_{p}}^{-1}\right)\right)
$$

admettant une norme $G_{p}$-invariante se trouvent dans le quotient localement algébrique $L(\lambda) \otimes_{L} \operatorname{Ind} \frac{G_{p}}{\bar{B}_{p}}\left(\Sigma_{\infty}\left(\delta_{B_{p}}^{-1}\right)\right)$. Ainsi le morphisme (3.13) se factorise par $L(\lambda) \otimes_{L} \operatorname{Ind} \bar{B}_{B_{p}}^{G_{p}}\left(\Sigma_{\infty}\left(\delta_{B_{p}}^{-1}\right)\right)$. On obtient au final un isomorphisme

$$
\operatorname{Hom}_{T_{p}}\left(\Sigma, J_{B_{p}}\left(\Pi_{\infty}^{R_{\infty}-\mathrm{an}}\left[\mathfrak{p}_{z}\right]\right)\right) \simeq \operatorname{Hom}_{G_{p}}\left(L(\lambda) \otimes_{L} \operatorname{Ind}_{\bar{B}_{p}}^{G_{p}}\left(\Sigma_{\infty}\left(\delta_{B_{p}}^{-1}\right)\right), \Pi_{\infty}\left[\mathfrak{p}_{z}\right]\right)
$$

Comme Ind $\bar{B}_{\bar{B}_{p}}^{G_{p}}\left(\Sigma_{\infty}\left(\delta_{B_{p}}^{-1}\right)\right)$ est une extension de longueur finie de représentations non ramifiées irréductibles, il existe un $\mathcal{H}:=L\left[K_{p} \backslash G_{p} / K_{p}\right]$-module de dimension finie $\mathcal{M}^{\text {lc }}$ et un isomorphisme $G_{p}$-équivariant entre $\operatorname{Ind}_{\bar{B}_{p}}^{G_{p}}\left(\Sigma_{\infty}\left(\delta_{B_{p}}^{-1}\right)\right)$ et $\operatorname{ind}_{K_{p}}^{G_{p}}(1) \otimes_{\mathcal{H}} \mathcal{M}^{\text {lc }}$. En utilisant par ailleurs [56, Lem. 1.4], on a donc au final un isomorphisme

$$
\operatorname{Hom}_{T_{p}}\left(\Sigma, J_{B_{p}}\left(\Pi_{\infty}^{R_{\infty}-\mathrm{an}}\left[\mathfrak{p}_{z}\right]\right)\right) \simeq \operatorname{Hom}_{\mathcal{H}(\lambda)}\left(\mathcal{M}^{\mathrm{lc}}, \Pi_{\infty}(\lambda)\left[\mathfrak{p}_{z}\right]\right)
$$

Or d'après [15, Lemma 4.16] dont la preuve s'étend essentiellement verbatim à notre situation, l'image de $R_{\infty}$ dans l'anneau des endomorphismes de $\Pi_{\infty}(\lambda)$ est un anneau réduit. Rappelons que l'action de $R_{\infty}$ sur $\Pi_{\infty}(\lambda)$ se factorise à travers

$$
R_{\infty}(\lambda):=R_{\bar{\rho}_{p}}^{\square, \mathbf{k}-\mathrm{cr}} \llbracket x_{1}, \ldots, x_{g} \rrbracket
$$

où $\lambda=\left(\lambda_{\tau, i}\right) \in \prod_{v \in S_{p}}\left(\mathbb{Z}^{n}\right)^{\left[F_{v}^{+}: \mathbb{Q}_{p}\right]}$ est lié à $\mathbf{k} \in \prod_{v \in S_{p}}\left(\mathbb{Z}^{n}\right)^{\left[F_{v}^{+}: \mathbb{Q}_{p}\right]}$ par la relation usuelle $\lambda_{\tau, i}=k_{\tau, i}+(i-1)$. Comme $\Pi_{\infty}(\lambda)^{\prime}$ est un $S_{\infty}\left[\frac{1}{p}\right]$-module projectif de type fini par loc. cit., on déduit de [17, Lem. 3.10.(b)] qu'il existe un ouvert $W$ de $\operatorname{Spec}\left(S_{\infty}\left[\frac{1}{p}\right]\right)$ tel que pour tout point fermé $z \in W$, le module $\Pi_{\infty}(\lambda)^{\prime} \otimes k(z)$ soit un $R_{\infty}(\lambda)$-module semi-simple. Posons alors $V_{\lambda}=(W \times\{\lambda\}) \cap V$. Si $z \in W$, la représentation $\Pi_{\infty}(\lambda)\left[\mathfrak{p}_{z}\right]$ est donc un $R_{\infty}$-module semi-simple d'après ce qui précède. De plus, l'action de $\mathcal{H}(\lambda)$ sur $\Pi_{\infty}(\lambda)$ se factorise par l'action de $R_{\infty}(\lambda)$, via le morphisme $\mathcal{H}(\lambda) \rightarrow R_{\bar{\rho}_{p}}^{\square, \mathbf{k} \text {-cr }}$ déjà considéré dans la preuve de la Proposition 3.15. En particulier, comme $\Pi_{\infty}(\lambda)\left[\mathfrak{p}_{z}\right]$ est un $R_{\infty}(\lambda)$-module semi-simple de dimension finie sur $L$, c'est également un $\mathcal{H}(\lambda)$-module semi-simple. On en déduit donc que tout morphisme de $\mathcal{M}^{\text {lc }}$ dans $\Pi_{\infty}(\lambda)\left[\mathfrak{p}_{z}\right]$ se factorise à travers le plus grand quotient semi-simple de $\mathcal{M}^{\text {lc }}$. Notons $\mathcal{M}_{\mathrm{ss}}^{\text {lc }}$ ce plus grand quotient. Alors ind $K_{K_{p}}^{G_{p}}(1) \otimes_{\mathcal{H}(1)} \mathcal{M}_{\mathrm{ss}}^{\text {lc }}$ est le plus grand quotient semi-simple de $\left.\operatorname{Ind}{\frac{G_{p}}{B_{p}}}_{\left(\Sigma_{\infty}\right.}\left(\delta_{B_{p}}^{-1}\right)\right)$, il est donc de la forme $\operatorname{Ind} \frac{G_{p}}{\bar{B}_{p}}\left(\Sigma_{\mathrm{sS}}\left(\delta_{B_{p}}^{-1}\right)\right)$, où $\Sigma_{\mathrm{ss}}$ est le plus grand quotient semi-simple de $\Sigma_{\infty}$ (utiliser l'exactitude du foncteur de Jacquet sur les représentations lisses). On obtient donc au final

$$
\operatorname{Hom}_{T_{p}}\left(\Sigma, J_{B_{p}}\left(\Pi_{\infty}^{R_{\infty}-\operatorname{an}}\left[\mathfrak{p}_{z}\right]\right)\right) \simeq \operatorname{Hom}_{T_{p}}\left(\delta_{\lambda} \otimes_{L} \Sigma_{\mathrm{ss}}, J_{B_{p}}\left(\Pi_{\infty}^{R_{\infty}-\mathrm{an}}\left[\mathfrak{p}_{z}\right]\right)\right)
$$

Comme $\Sigma$ est une sous-représentation de $J_{B_{p}}\left(\Pi_{\infty}^{R}-\right.$ an $\left.\left[\mathfrak{p}_{z}\right]\right)$, on en conclut que $\Sigma$ est en fait une représentation semi-simple de $T_{p}^{+}$. 
3.6. Composantes irréductibles de $X_{p}(\bar{\rho})$. Notons $\iota$ l'automorphisme

$$
\operatorname{Id}_{\mathfrak{X}_{\overline{\bar{\rho}_{p}}}} \times\left(\iota_{F_{v}^{+}}\right)_{v \in S_{p}}: \mathfrak{X}_{\bar{\rho}_{p}}^{\square} \times \widehat{T}_{p, L} \longrightarrow \mathfrak{X}_{\bar{\rho}_{p}}^{\square} \times \widehat{T}_{p, L} .
$$

Théorème 3.20. L'immersion

$$
X_{p}(\bar{\rho}) \longrightarrow \operatorname{Spf}\left(R_{\infty}\right)^{\text {rig }} \times \widehat{T}_{p, L} \simeq \mathfrak{X}_{\bar{\rho}_{p}}^{\square} \times \mathfrak{X}_{\bar{\rho}^{p}}^{\square} \times \mathbb{U}^{g} \times \widehat{T}_{p, L}
$$

induit un isomorphisme d'espaces analytiques rigides entre $X_{p}(\bar{\rho})$ et une union de composantes irréductibles du sous-espace analytique fermé $\iota\left(X_{\text {tri }}^{\square}\left(\bar{\rho}_{p}\right)\right) \times \mathfrak{X}_{\bar{\rho}^{p}}^{\square} \times \mathbb{U}^{g}$ muni de sa structure de sous-espace analytique fermé réduit.

Démonstration. Nous allons prouver que le sous-espace analytique fermé de l'espace rigide $\mathfrak{X}_{\bar{\rho}_{p}}^{\square} \times \mathfrak{X}_{\bar{\rho}^{p}}^{\square} \times \mathbb{U}^{g} \times \widehat{T}_{p, L}$ sous-jacent à $X_{p}(\bar{\rho})$ est en réalité contenu dans $\iota\left(X_{\text {tri }}^{\square}\left(\bar{\rho}_{p}\right)\right) \times \mathfrak{X}_{\bar{\rho}^{p}}^{\square} \times \mathbb{U}^{g}$. Comme ces deux espaces sont équidimensionnels de même dimension et que $X_{p}(\bar{\rho})$ est réduit, on en conclut que $X_{p}(\bar{\rho})$ s'identifie bien en tant qu'espace analytique rigide à une union de composantes irréductibles de $\iota\left(X_{\text {tri }}^{\square}\left(\bar{\rho}_{p}\right)\right) \times \mathfrak{X}_{\bar{\rho}^{p}}^{\square} \times \mathbb{U}^{g}$, cette union étant munie de sa structure réduite de sous-espace analytique fermé.

Par définition de $\iota$ et des variétés $X_{p}(\bar{\rho})$ et $X_{\text {tri }}^{\square}\left(\bar{\rho}_{p}\right)$, les points très classiques de $X_{p}(\bar{\rho})$ appartiennent aussi à $\iota\left(X_{\text {tri }}^{\square}\left(\bar{\rho}_{p}\right)\right) \times \mathfrak{X}_{\bar{\rho}^{p}}^{\square} \times \mathbb{U}^{g}$. D'après le Théorème 3.18 , ils forment une partie dense au sens de Zariski de $X_{p}(\bar{\rho})$. Comme $\iota\left(X_{\text {tri }}^{\square}\left(\bar{\rho}_{p}\right)\right) \times \mathfrak{X}_{\bar{\rho}^{p}}^{\square} \times \mathbb{U}^{g}$ est une partie analytique fermée de $\mathfrak{X}_{\bar{\rho}_{p}}^{\square} \times \mathfrak{X}_{\bar{\rho}^{p}}^{\square} \times \mathbb{U}^{g} \times \widehat{T}_{p, L}$, on obtient bien l'inclusion recherchée.

Une composante irréductible de l'espace $\iota\left(X_{\text {tri }}^{\square}\left(\bar{\rho}_{p}\right)\right) \times \mathfrak{X}_{\bar{\rho}^{p}}^{\square} \times \mathbb{U}^{g}$ est un produit de la forme $\iota(X) \times \mathfrak{X}^{p} \times \mathbb{U}^{g}$, où $X$ est une composante irréductible de $X_{\text {tri }}^{\square}\left(\bar{\rho}_{p}\right)$ et $\mathfrak{X}^{p}$ une composante irréductible de $\mathfrak{X}_{\bar{\rho}^{p}}^{\square}$. Il nous paraît raisonnable de conjecturer qu'à $\mathfrak{X}^{p}$ fixée, les composantes irréductibles $X$ de $X_{p}(\bar{\rho})$ de la forme $\iota(X) \times \mathfrak{X}^{p} \times \mathbb{U}^{g}$ ne dépendent pas des choix globaux que nous avons faits, c'est-à-dire de $G$, de $U^{p}$ et du système de Taylor-Wiles intervenant dans la construction de $\Pi_{\infty}$.

Définition 3.21. Soit $\mathfrak{X}^{p}$ une composante irréductible de $\mathfrak{X}_{\bar{\rho}^{p}}^{\square}$. On dit qu'une composante irréductible $X$ de $X_{\mathrm{tri}}^{\square}\left(\bar{\rho}_{p}\right)$ est $\mathfrak{X}^{p}$-automorphe si $\iota(X) \times \mathfrak{X}^{p} \times \mathbb{U}^{g}$ est une composante irréductible de $X_{p}(\bar{\rho})$.

Conjecture 3.22. Une composante irréductible $X$ de $X_{\text {tri }}^{\square}\left(\bar{\rho}_{p}\right)$ est $\mathfrak{X}^{p}$-automorphe si et seulement si $X \cap U_{\text {tri }}^{\square}\left(\bar{\rho}_{p}\right)^{\text {reg }}$ contient un point cristallin.

Remarque 3.23. (i) Si $S \backslash S_{p}=\left\{v_{1}\right\}$, où v $v_{1}$ est une place de $F^{+}$comme en [15, § 2.3], et $U^{p}$ est choisi comme dans loc. cit., alors $\mathfrak{X}_{\bar{\rho}^{p}}^{\square}$ est lisse et irréductible.

(ii) Il résulte du Théorème 3.18 (cf. la fin de la preuve) qu'une composante irréductible $\mathfrak{X}^{p}$ automorphe de $X_{\mathrm{tri}}^{\square}\left(\bar{\rho}_{p}\right)$ contient toujours un tel point cristallin. La Conjecture 3.22 peut donc se reformuler comme suit : les composantes irréductibles de $X_{p}(\bar{\rho})$ sont exactement les composantes $\iota(X) \times \mathfrak{X}^{p} \times \mathbb{U}^{g}$ où $\mathfrak{X}^{p}$ est une composante irréductible de $\mathfrak{X}_{\bar{\rho}^{p}}^{\square}$ et $X$ une composante irréductible de $X_{\mathrm{tri}}^{\square}\left(\bar{\rho}_{p}\right)$ telle que $X \cap U_{\mathrm{tri}}^{\square}\left(\bar{\rho}_{p}\right)^{\text {reg }}$ contient un point cristallin de poids de Hodge-Tate deux-à-deux distincts.

Dans ce qui suit, si $\lambda \in\left(\mathbb{Z}^{n}\right)^{\operatorname{Hom}\left(F_{v}^{+}, L\right)}$, on note $\sigma(\lambda)$ la représentation algébrique de $\mathrm{GL}_{n, F_{v}^{+}}$de plus haut poids $\lambda$, et on note de même sa restriction à $\mathrm{GL}_{n}\left(\mathcal{O}_{F_{v}^{+}}\right)$. Fixons un poids $\mathbf{k} \in \prod_{v \in S_{p}}\left(\mathbb{Z}^{n}\right)^{\operatorname{Hom}\left(F_{v}^{+}, L\right)}$ strictement dominant. On définit alors une représentation $\sigma_{\mathbf{k}}$ de $K_{p}=\prod_{v \in S_{p}} G\left(F_{v}^{+}\right)$en posant

$$
\sigma_{\mathbf{k}}=\bigotimes_{v \in S_{p}} \sigma\left(\left(k_{\tau, i}+i-1\right)_{\tau \in \operatorname{Hom}\left(F_{v}^{+}, L\right), 1 \leq i \leq n}\right) .
$$


La conjecture suivante apparaît essentiellement dans l'article [29].

Conjecture 3.24. Soit $\mathfrak{X}^{p}$ une composante irréductible de $\mathfrak{X}_{\bar{\rho}^{p}}^{\square}$. Soit $\mathbf{k} \in \prod_{v \in S_{p}}\left(\mathbb{Z}^{n}\right)^{\left[F_{v}^{+}: \mathbb{Q}_{p}\right]}$ strictement dominant, l'espace $\operatorname{Spf}\left(R_{\bar{\rho}_{p}}^{\square, \mathbf{k}-\mathrm{cr}}\right)^{\mathrm{rig}} \times \mathfrak{X}^{p} \times \mathbb{U}^{g}$ est contenu dans le support du $R_{\infty}$-module $\operatorname{Hom}_{K_{p}}\left(\sigma_{\mathbf{k}}, \Pi_{\infty}\right)^{\prime}$.

Remarque 3.25. En fait lorsque $S=S_{p} \sqcup\left\{v_{1}\right\}$ où $v_{1}$ est une place de $F^{+}$vérifiant les conditions de [29, §5.3], d'après [29, Thm. 5.5.2], la Conjecture 3.24 est équivalente à la Conjecture de Breuil-Mézard raffinée dans les cas cristallins énoncée en [29, Conj. 4.1.6].

Proposition 3.26. La conjecture 3.24 implique la conjecture 3.22.

Démonstration. Soit $\mathfrak{U}^{p}$ l'ensemble des points lisses de $\mathfrak{X}^{p}$. Comme $\mathfrak{X}^{p}$ est réduit, il s'agit d'un ouvert de Zariski, Zariski-dense dans $\mathfrak{X}^{p}$ par la Proposition 2.3. Soit $X$ une composante irréductible de $X_{\text {tri }}^{\square}\left(\bar{\rho}_{p}\right)$. Supposons que $X \cap U_{\text {tri }}^{\square}\left(\bar{\rho}_{p}\right)^{\text {reg }}$ contienne un point $x$ cristallin. Son image $\omega(x) \in \widehat{T}_{p, L}^{0}$ est donc un caractère algébrique. D'après le Théorème 2.6, l'application $\omega$ est lisse au point $x$. Il existe donc un voisinage affinoïde connexe $U \subset U_{\text {tri }}^{\square}\left(\bar{\rho}_{p}\right)^{\text {reg }} \cap X$ de $x$ dont l'image par $\omega$ est un ouvert affinoïde de $\widehat{T}_{p, L}^{0}$. En effet, d'après [1, Prop. 6.4.22], l'application $\omega$ est plate en $x$. Quitte à se restreindre à des voisinages affinoïdes de $x$ et $\omega(x)$, on peut voir $\omega$ comme l'analytifié d'un morphisme plat entre schémas formels $\mathcal{X} \rightarrow \mathcal{Y}$. D'après [1, Prop. 5.10.14], un tel morphisme se factorise en $\mathcal{X} \stackrel{g}{\rightarrow} \mathcal{U} \stackrel{j}{\rightarrow} \mathcal{Y}$ où $j$ est une immersion ouverte et $g$ est fidèlement plate, on conclut alors en observant qu'une application fidèlement plate est surjective (on peut aussi appliquer [36, Prop. 1.7.8]).

Comme de plus l'ensemble des caractères algébriques strictement dominants dans $\widehat{T}_{p, L}^{0}$ s'accumule en l'ensemble des caractères algébriques, il existe $\mathbf{k}$ strictement dominant tel que $\delta_{\mathbf{k}} \in \omega(U)$. Fixons un tel k. D'après [35, Cor. 2.7], on peut choisir le point $y=\left(y_{1}, y_{2}\right) \in U \times \mathfrak{U}^{p}$ tel que $\omega\left(y_{1}\right)=\delta_{\mathbf{k}}$ et $y_{1} \in \mathfrak{X}_{\bar{\rho}_{p}}^{\mathbf{k}-\text { cr }}$. Soit $\mathfrak{p}$ un ideal maximal de $R_{\infty}\left[\frac{1}{p}\right]$ dont l'intersection avec $R^{\text {loc }}\left[\frac{1}{p}\right]$ soit l'idéal du point $y$.

La Conjecture 3.24 implique alors que $\operatorname{Hom}_{K_{p}}\left(\sigma_{\mathbf{k}}, \Pi_{\infty}[\mathfrak{p}]\right) \neq 0$. On déduit alors de $[15$, Lemma 4.16], dont la preuve s'étend verbatim à notre situation, que l'on a un isomorphisme

$$
\operatorname{Hom}_{K_{p}}\left(\sigma_{\mathbf{k}}, \Pi_{\infty}[\mathfrak{p}]\right) \simeq \operatorname{Hom}_{G_{p}}\left(\sigma_{\mathbf{k}} \otimes_{L} \pi(\mathfrak{p}), \Pi_{\infty}[\mathfrak{p}]\right)
$$

où $\pi(\mathfrak{p})$ désigne la représentation lisse irréductible de $G_{p}$, de la série principale, telle que $\pi(\mathfrak{p}) \otimes|\operatorname{det}|^{\frac{1-n}{2}}$ corresponde au point $\mathfrak{p} \cap R_{\bar{\rho}_{p}}^{\square, \mathbf{k}-\mathrm{cr}}$ par la correspondance de Langlands locale (i.e. par la correspondance de Langlands locale appliquée en chaque place $v$ divisant $p)$. On déduit de ceci et du calcul du module de Jacquet de $\sigma_{\mathbf{k}} \otimes_{L} \pi(\mathfrak{p})$ que tous les points de $\left\{\left(\iota\left(y_{1}\right), y_{2}\right)\right\} \times \mathbb{U}^{g}$ sont dans le support de $\mathcal{M}_{\infty}$. Comme $\{y\} \times \mathbb{U}^{g}$ est un ensemble irréductible de points lisses de $X_{\text {tri }}^{\square}\left(\bar{\rho}_{p}\right) \times \mathfrak{X}_{\bar{\rho}^{p}}^{\square} \times \mathbb{U}^{g}$, il est inclus dans une unique composante irréductible de cet espace. Comme d'après le Théorème 3.20 le support de $\mathcal{M}_{\infty}$ est une union de composantes irréductibles, on en déduit que l'image par $\iota$ de cette composante irréductible est incluse dans $X_{p}(\bar{\rho})$. Remarquons à présent que l'ouvert $U$ est connexe, donc cette composante irréductible contient également le point $x$ de depart. Comme $x$ est également un point lisse de $X_{\text {tri }}^{\square}\left(\bar{\rho}_{p}\right) \times \mathfrak{X}_{\bar{\rho}^{p}}^{\square} \times \mathbb{U}^{g}$, l'ensemble $X \times \mathfrak{X}^{p} \times \mathbb{U}^{g}$ est l'unique composante irréductible contenant ce point, on a donc au final $\iota(X) \times \mathfrak{X}^{p} \times \mathbb{U}^{g} \subset X_{p}(\bar{\rho})$.

Proposition 3.27. La conjecture 3.22 implique la conjecture 3.24. 
Démonstration. Soit $\mathfrak{X}^{p}$ une composante irréductible de $\mathfrak{X}_{\bar{\rho}^{p}}^{\square}$ et $\delta_{\mathbf{k}}$ un caractère algébrique strictement dominant de $T_{p}$. Soit $\mathfrak{X}_{p}$ une composante irréductible de $\mathfrak{X}_{\bar{\rho}_{p}}^{\square, \mathbf{k}-\text { cr }}$. D'après [19, Lem. 4.4], on peut choisir

$$
x_{p}=\left(x_{v}\right)_{v \in S_{p}} \in \mathfrak{X}_{p} \subset \mathfrak{X}_{\bar{\rho}_{p}, \mathbf{k}-\mathrm{cr}} \simeq \prod_{v \in S_{p}} \mathfrak{X}_{\bar{\rho}_{\tilde{v}}}^{\square, k_{v}-\mathrm{cr}}
$$

tel que la représentation cristalline de $\mathcal{G}_{F_{\tilde{v}}}$ associée à $x_{v}$ soit générique pour tout $v \in S_{p}$ au sens de la Définition 2.8. Soit $\delta$ le caractère $\delta_{\mathbf{k}} \eta$ de $T_{p}$ où $\eta$ est le caractère lisse non ramifié de la forme $\prod_{v \in S_{p}} \eta_{v}$ avec $\eta_{v}$ l'unique caractère lisse non ramifié de $T_{v}$ envoyant $\left(x_{1}, \ldots, x_{n}\right)$ sur $\prod_{i=1}^{n} \varphi_{i}^{v\left(x_{i}\right)}$ où $\left(\varphi_{1}, \ldots, \varphi_{n}\right)$ est une suite de valeurs propres du Frobenius cristallin de $x_{v}$. Le Lemme 2.11 implique en particulier que le point $\left(x_{p}, \delta\right)$ appartient à $U_{\text {tri }}^{\square}(\bar{r})^{\text {reg }}$. Sous la Conjecture 3.22, on a donc $\left\{\left(x_{p}, \delta\right)\right\} \times \mathfrak{X}^{p} \times \mathbb{U}^{g} \subset X_{p}(\bar{\rho})$. Notons temporairement

$$
\mathcal{F}_{\bar{B}_{p}}^{G_{p}}(\delta):=\mathcal{F}_{\bar{B}_{p}}^{G_{p}}\left(U\left(\mathfrak{g}_{L}\right) \otimes_{U\left(\overline{\mathfrak{b}}_{L}\right)}(-\lambda), \eta \delta_{B_{p}}^{-1}\right),
$$

où $\lambda=\left(k_{\tau, i}+i-1\right)_{\tau \in \operatorname{Hom}\left(F_{v}^{+}, L\right), 1 \leq i \leq n}$. Fixons $x \in\left\{\left(x_{p}, \delta\right)\right\} \times \mathfrak{X}^{p} \times \mathbb{U}^{g}$. Comme $x \in X_{p}(\bar{\rho})$, il existe un morphisme non trivial de $\mathcal{F}_{\bar{B}_{p}}^{G_{p}}(\delta)$ vers $\Pi_{\infty}\left[\mathfrak{p}_{x}\right]^{\text {an }}$. Pour prouver que le point $y$, image de $x$ dans $\operatorname{Spf}\left(R_{\infty}\right)^{\text {rig }}$ est dans le support de $\operatorname{Hom}_{K_{p}}\left(\sigma_{\delta}, \Pi_{\infty}\right)^{\prime}$, il suffit de prouver que ce morphisme se factorise par le quotient localement algébrique de $\mathcal{F}_{\bar{B}_{p}}^{G_{p}}(\delta)$. Or si ce n'était pas le cas, la description des facteurs de Jordan-Hölder de $\mathcal{F}_{\bar{B}_{p}}^{G_{p}}(\delta)$ (cf. [11, Cor. 4.6]) implique qu'il existerait un caractère localement algébrique non dominant dans le socle de la $T_{p}$-représentation $J_{B_{p}}\left(\Pi_{\infty}\left[\mathfrak{p}_{x}\right]^{\text {an }}\right)$ et donc un point de $X_{p}(\bar{\rho})$ dont la projection dans $X_{\mathrm{tri}}^{\square}\left(\bar{\rho}_{p}\right)$ est de la forme $\left(x_{p}, \delta^{\prime}\right)$ avec $\delta^{\prime}$ un caractère localement algébrique de poids non dominant, ce qui contredit le Lemme 2.11 .

\section{Application aux variétés de Hecke}

4.1. L'espace des représentations galoisiennes de pente finie. Rappelons que l'on a défini au $\S 2.4$ le Banach $p$-adiques $\widehat{S}\left(U^{p}, L\right)$ de niveau modéré $U^{p}$.

Soit $S, \bar{\rho}$ et $\mathcal{S}$ comme aux $\S 2.4$ et $\S 3.2$, et $\mathfrak{X}_{\bar{\rho}, \mathcal{S}}=\operatorname{Spf}\left(R_{\bar{\rho}, \mathcal{S}}\right)^{\text {rig. }}$. On désigne $\operatorname{par} Y\left(U^{p}, \bar{\rho}\right)$ la sous-variété analytique rigide de $\mathfrak{X}_{\bar{\rho}, \mathcal{S}} \times \widehat{T}_{p, L}$ support du faisceau cohérent $\mathcal{M}_{U^{p}}$ défini par $J_{B_{p}}\left(\widehat{S}\left(U^{p}, L\right)_{\mathfrak{m}}^{\mathrm{an}}\right)$. Il s'agit de la définition de la variété de Hecke ([16]) proposée dans [27]. Les méthodes de $[17, \S 3.8]$ permettent de montrer qu'il s'agit d'un espace rigide analytique réduit.

Notons $R_{\bar{\rho}_{S}}^{\square}$ l'algèbre $\widehat{\otimes}_{v \in S} R_{\bar{\rho}_{\bar{v}}}^{\square}$ et $R_{\bar{\rho}_{S}}^{\bar{\square}}$ l'algèbre $\widehat{\otimes}_{v \in S} R_{\bar{\rho}_{\bar{v}}}^{\bar{\Sigma}}$ et $\mathfrak{X}_{\bar{\rho}_{S}}^{\square}=\operatorname{Spf}\left(R_{\bar{\rho}_{S}}^{\bar{\square}}\right)^{\text {rig. On a }}$ alors une décomposition en produit $\mathfrak{X}_{\bar{\rho}_{S}}^{\square} \simeq \mathfrak{X}_{\bar{\rho}_{p}}^{\square} \times \mathfrak{X}_{\bar{\rho}^{p}}^{\square}$ et on définit

$$
X_{\text {tri }}^{\square}\left(\bar{\rho}_{S}\right)=X_{\text {tri }}^{\square}\left(\bar{\rho}_{p}\right) \times \mathfrak{X}_{\bar{\rho}^{p}}^{\square} \subset \mathfrak{X}_{\bar{\rho}_{S}}^{\square} \times \widehat{T}_{p, L} .
$$

On désigne par $X_{\text {tri }}^{\square}\left(\bar{\rho}_{S}\right)^{\text {aut }}$ le sous-espace analytique rigide fermé de $X_{\text {tri }}^{\square}\left(\bar{\rho}_{p}\right) \times \mathfrak{X}_{\bar{\rho}^{p}}^{\square}$ union des composantes irréductibles $X \times \mathfrak{X}^{p}$, où $X$ parcourt l'ensemble des composantes irréductibles $\mathfrak{X}^{p}$-automorphes (cf. Déf. 3.21) et $\mathfrak{X}^{p}$ parcourt l'ensemble des composantes irréductibles de $\mathfrak{X}_{\bar{\rho}^{p}}^{\square}$. L'espace $X_{\text {tri }}^{\square}\left(\bar{\rho}_{S}\right)^{\text {aut }}$ dépend donc des choix faits dans le Théorème 3.4 .

Ce même choix donne lieu à un morphisme local de $\mathcal{O}_{L}$-algèbres locales $R_{\overline{\bar{\rho}} S}^{\overline{\bar{\square}}} \rightarrow R_{\bar{\rho}, \mathcal{S}}$ obtenu par composition de l'inclusion naturelle $R_{\overline{\bar{\rho}}_{S}}^{\bar{C}_{S}} \hookrightarrow R_{\infty}$ avec le morphisme surjectif 
$R_{\infty} \rightarrow R_{\bar{\rho}, \mathcal{S}}$ du Théorème 3.4. Passant aux espaces analytiques rigides associés, on obtient une application $\mathfrak{X}_{\bar{\rho}, \mathcal{S}} \rightarrow \mathfrak{X}_{\bar{\rho}_{S}}^{\square}$.

Le but de cette partie est de comparer les espaces $Y\left(U^{p}, \bar{\rho}\right)$ et

$$
X(\bar{\rho})_{\mathrm{fs}}:=\iota\left(X_{\text {tri }}^{\square}\left(\bar{\rho}_{S}\right)\right) \times_{\mathfrak{X}_{\bar{\rho}}^{\square}} \mathfrak{X}_{\bar{\rho}, \mathcal{S}} .
$$

Ce dernier espace est introduit dans [33] (avec fs pour « finite slope »).

Remarque 4.1. On a $R_{\bar{\rho}, \mathcal{S}}=R_{\bar{\rho}, S} \widehat{\otimes}_{R_{\bar{\rho}_{S}}} R_{\bar{\rho}_{S}}^{\bar{\square}}$. En particulier on a

$$
\mathfrak{X}_{\bar{\rho}, \mathcal{S}}=\operatorname{Spf}\left(R_{\bar{\rho}, S}\right)^{\text {rig }} \times_{\operatorname{Spf}\left(R_{\bar{\rho}_{S}}\right)^{\text {rig }}} \operatorname{Spf}\left(R_{\bar{\rho}_{S}}^{\bar{\square}}\right)^{\text {rig }} .
$$

Chaque $R_{\bar{\rho}_{\tilde{v}}}^{\bar{v}}$ est le plus grand quotient réduit et sans $p$-torsion de $R_{\bar{\rho}_{\tilde{v}}}^{\square}$. On déduit alors de la preuve de [43, Lem. 3.4.12] que $R_{\bar{\rho}_{S}}^{\bar{D}}$ est le plus grand quotient réduit et sans p-torsion de $R_{\bar{\rho}_{S}}^{\square}$. Ainsi $\operatorname{Spf}\left(R_{\bar{\rho}_{S}}^{\square}\right)^{\text {rig }}$ et $\operatorname{Spf}\left(R_{\bar{\rho}_{S}}^{\overline{\rho_{S}}}\right)^{\text {rig }}$ ont même espace sous-jacent, et donc les espaces analytiques rigides $\operatorname{Spf}\left(R_{\bar{\rho}, S}\right)^{\text {rig }}$ et $\mathfrak{X}_{\bar{\rho}, \mathcal{S}}$ ont même espace sous-jacent.

Nous aurons également besoin des notations suivantes. L'espace analytique rigide $\mathfrak{X}_{\bar{\rho} p}^{\square}$, étant un produit d'espaces analytiques rigides réduits, est réduit. L'ensemble de ses points lisses forme donc un ouvert au sens de Zariski qui est non vide et Zariski-dense. Notons $\mathfrak{X}_{\bar{\rho}^{p}}^{\square \text {,reg }}$ cet ouvert. Soit

$$
X(\bar{\rho})_{\mathrm{fs}}^{\mathrm{reg}}=\mathfrak{X}_{\bar{\rho}, \mathcal{S}} \times_{\mathfrak{X}_{\bar{\rho} p}^{\square} \times \mathfrak{X}_{\bar{\rho} p}^{\square}}\left(\iota\left(U_{\text {tri }}^{\square}\left(\bar{\rho}_{p}\right)^{\mathrm{reg}}\right) \times \mathfrak{X}_{\bar{\rho}^{p}}^{\square, \mathrm{reg}}\right) .
$$

Il s'agit d'un ouvert au sens de Zariski de $X(\bar{\rho})_{\mathrm{fs}}$. On définit alors $Y\left(U_{p}, \bar{\rho}\right)^{\text {reg }}$ le sousespace analytique ouvert (au sens de Zariski) de $Y\left(U^{p}, \bar{\rho}\right)$ dont l'espace sous-jacent est donné par $Y\left(U^{p}, \bar{\rho}\right) \cap X(\bar{\rho})_{\mathrm{fs}}^{\text {reg }}$ et muni de la structure d'espace analytique rigide induite par celle de $Y\left(U^{p}, \bar{\rho}\right)$.

Remarquons que comme $X_{\text {tri }}^{\square}\left(\bar{\rho}_{S}\right)$ est un sous-espace analytique fermé de $\mathfrak{X}_{\bar{\rho}_{S}}^{\square} \times \widehat{T}_{p, L}$, l'espace $X(\bar{\rho})_{\mathrm{fs}}$ est un sous-espace analytique fermé de $\mathfrak{X}_{\bar{\rho}, \mathcal{S}} \times \widehat{T}_{p, L}$.

Théorème 4.2. Dans $\mathfrak{X}_{\bar{\rho}, \mathcal{S}} \times \widehat{T}_{p, L}$, on a une égalité d'ensembles fermés analytiques $Y\left(U^{p}, \bar{\rho}\right)=\mathfrak{X}_{\bar{\rho}, \mathcal{S}} \times_{\mathfrak{X}_{\bar{\rho}}^{\square}} \iota\left(X_{\text {tri }}^{\square}\left(\bar{\rho}_{S}\right)^{\text {aut }}\right)$.

Démonstration. L'isomorphisme $R_{\bar{\rho}, \mathcal{S}} \simeq R_{\infty} / \mathfrak{a}$ se traduit, en termes d'espaces analytiques

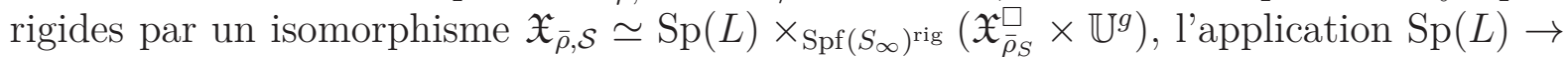
$\operatorname{Spf}\left(S_{\infty}\right)^{\text {rig }}$ provenant de la spécialisation $y_{i} \mapsto 0$, ce qui nous permet d'identifier $\mathfrak{X}_{\bar{\rho}, \mathcal{S}}$ à un sous-espace analytique rigide fermé de $\mathfrak{X}_{\infty} \times \widehat{T}_{p, L}$. Par exactitude à gauche du foncteur $J_{B_{p}}$ ainsi que du foncteur de passage aux vecteurs localement analytiques, on a $J_{B_{p}}\left(\Pi_{\infty}^{R_{\infty}-\mathrm{an}}\right)[\mathfrak{a}] \simeq J_{B_{p}}\left(\Pi_{\infty}[\mathfrak{a}]^{\text {an }}\right)$. L'isomorphisme (3.4) montre alors que le faisceau cohérent $\mathcal{M}_{U^{p}}$ sur $\mathfrak{X}_{\bar{\rho}, \mathcal{S}} \times \widehat{T}_{p, L}^{0}$ est l'image inverse du faisceau cohérent $\mathcal{M}_{\infty}$ sur $\mathfrak{X}_{\infty} \times \widehat{T}_{p, L}^{0}$. On en conclut que le support de $\mathcal{M}_{U^{p}}$ en tant que partie fermée analytique coïncide avec l'image inverse du support de $\mathcal{M}_{\infty}$, c'est-à-dire est le fermé analytique sous-jacent

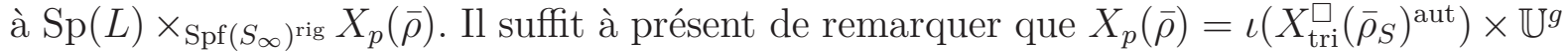
par le Théorème 3.20 et que

$$
\begin{aligned}
\operatorname{Sp}(L) \times_{\operatorname{Spf}\left(S_{\infty}\right)^{\text {rig }}}\left(\iota\left(X_{\text {tri }}^{\square}\left(\bar{\rho}_{S}\right)^{\text {aut }}\right) \times \mathbb{U}^{g}\right) & \simeq \operatorname{Sp}(L) \times_{\operatorname{Spf}\left(S_{\infty}\right)^{\text {rig }}} \mathfrak{X}_{\bar{\rho}_{S}}^{\square} \times_{\mathfrak{X}_{\bar{\rho}}^{\square}}\left(\iota\left(X_{\text {tri }}^{\square}\left(\bar{\rho}_{S}\right)^{\text {aut }}\right) \times \mathbb{U}^{g}\right) \\
& \simeq\left(\operatorname{Sp}(L) \times_{\operatorname{Spf}\left(S_{\infty}\right)^{\text {rig }}} \mathfrak{X}_{\infty}\right) \times_{\mathfrak{X}_{\bar{\rho}_{S}}^{\square}} \iota\left(X_{\text {tri }}^{\square}\left(\bar{\rho}_{S}\right)^{\text {aut }}\right) \\
& \simeq \mathfrak{X}_{\bar{\rho}, \mathcal{S}} \times_{\mathfrak{X}_{\bar{\rho}}^{\square}} \iota\left(X_{\text {tri }}^{\square}\left(\bar{\rho}_{S}\right)^{\text {aut }}\right) .
\end{aligned}
$$


Corollaire 4.3. La conjecture 4.16 de [33] est vraie lorsque $\bar{\rho}$ vérifie les hypothèses des \$2.4 et 3.2.

Nous allons désormais montrer que la Conjecture 3.22 implique une partie des conjectures de [11]. Pour cela, plaçons dans le contexte de loc. cit. et supposons donc que $F_{v}^{+}=\mathbb{Q}_{p}$ pour tout $v \in S_{p}$. Remarquons qu'il n'y a pas de difficulté non typographique à étendre [11], ainsi que le Corollaire 4.4 ci-dessous, au cas général.

Soit $\pi$ une représentation automorphe de $G$ qui vérifie les conditions suivantes :

(i) $\pi^{U^{p}} \neq 0$, en particulier $\pi$ est non ramifiée hors de $S$;

(ii) pour $v \in S_{p}, \rho_{\pi, \tilde{v}}:=\left.\rho_{\pi}\right|_{\mathcal{G}_{\tilde{v}}}$ est cristalline générique au sens de [11, Déf. 5.2 ${ }^{3}$;

(iii) $\bar{\rho}_{\pi}$ est isomorphe à $\bar{\rho}$.

On note $\mathfrak{p}_{\pi}$ l'idéal maximal de $R_{\bar{\rho}, S}[1 / p]$ correspondant à $\rho_{\pi}$.

Corollaire 4.4. Supposons que $p \geq 2 n+2$ et $\left.\bar{\rho}\right|_{\mathcal{G}_{F\left(\zeta_{p}\right)}}$ est absolument irréductible. Alors la partie « existence 》 de la Conjecture 6.5 de [11] est vraie dans le cas cristallin. Plus précisément, pour tout caractère $\eta=\prod_{v \in S_{p}} \eta\left(w_{\tilde{v}}^{\text {alg }}, w_{\tilde{v}}\right)$ avec $w_{\tilde{v}}^{\text {alg }} \leq w_{\tilde{v}}^{\text {alg }}\left(w_{\tilde{v}}\right)$ (les notations sont celles de [11]), on a

$$
\operatorname{Hom}_{T_{p}}\left(\eta, J_{B_{p}}\left(\widehat{S}\left(U_{p}, L\right)^{\text {an }}\left[\mathfrak{p}_{\pi}\right]\right)\right) \neq 0 .
$$

Remarque 4.5. La condition (ii) peut être remplacée par la condition plus faible suivante :

(ii)' pour tout $v \in S_{p}, \rho_{\pi, \tilde{v}}$ est cristalline, pour tout plongement de $F_{v}^{+}$dans L, les poids de Hodge-Tate de $\rho_{\pi, \tilde{v}}$ sont deux-à-deux distincts, et si $\delta_{v}$ est un paramètre de $\rho_{\pi, \tilde{v}}$, on a $\delta_{v, i} \neq \delta_{v, j}$ pour $i \neq j$. En effet, par compatibilité local-global (cf. [14, Thm. 1.1]) on sait que $\pi_{v}$ est isomorphe à une représentation lisse de la forme $\operatorname{Ind}_{B_{v}}^{\mathrm{GL}_{n}\left(F_{v}^{+}\right)}\left(\eta_{v}\right)$ avec $\eta_{v, i} \eta_{v, j}^{-1} \neq|\cdot|_{F_{v}^{+}}^{i-j+1}$. C'est une conséquence du fait que $\pi_{v}$ est une représentation tempérée de $\mathrm{GL}_{n}\left(F_{v}^{+}\right)$(cf. [13, Thm. 1.2]) ainsi que de la classification des représentations tempérées non ramifiées ([44, §2.1 et 2.2$])$.

Démonstration. Soit $\left(\rho_{\pi}, \iota(\delta)\right) \in Y\left(U^{p}, \bar{\rho}\right)$ avec $\rho_{\pi}$ automorphe vérifiant les hypothèses (i) à (iv) de $[11, \S 6]$, en particulier $\rho_{\pi}$ est cristalline aux places de $S_{p}$ et $\delta$ localement algébrique strictement dominant, i.e. de la forme $\delta=\prod_{v \in S_{p}}\left(\delta_{\mathbf{k}_{v}} \eta_{v}\right)$ avec $\delta_{k_{v}}$ strictement dominant (au sens du début du $\S 2.2$ avec $K=F_{v}^{+}$) et $\eta_{v}$ un caractère lisse de $T_{v}$. Rappelons que, si $k_{v}=\left(k_{\tau, i}\right)_{1 \leq i \leq n, \tau: F_{v}^{+} \hookrightarrow L} \in\left(\mathbb{Z}^{n}\right)^{\operatorname{Hom}\left(F_{v}^{+}, L\right)}$, alors il existe $w_{v}=\left(w_{\tau}\right)_{\tau: F_{v}^{+} \hookrightarrow L} \in$ $S_{n}^{\operatorname{Hom}\left(F_{v}^{+}, L\right)}$ tel que $\delta_{w_{v}\left(k_{v}\right)} \eta_{v}$ est un paramètre de $\left.\rho_{\pi}\right|_{G_{F_{\tilde{v}}}}$ où $w_{v}\left(k_{v}\right)=\left(k_{\tau, w_{\tau}^{-1}(i)}\right)_{1 \leq i \leq n ; \tau: F_{v}^{+} \hookrightarrow L}$ (cf. par exemple [11, Lem. 6.4] où l'hypothèse $F_{v}^{+}=\mathbb{Q}_{p}$ ne joue aucun rôle). En particulier, le point $\left(\rho_{\pi},\left(\left.\rho_{\pi}\right|_{G_{F_{v}}}, \iota_{F_{v}}\left(\delta_{w_{v}\left(k_{v}\right)} \eta_{v}\right)\right)_{v \in S_{p}},\left(\left.\rho_{\pi}\right|_{G_{F_{\tilde{v}}}}\right)_{v \in S_{p}}\right)$ est dans

$$
\mathfrak{X}_{\bar{\rho}, \mathcal{S}} \times_{\mathfrak{X}_{\bar{\rho}}^{\square}}\left(\iota\left(U_{\text {tri }}^{\square}\left(\bar{\rho}_{p}\right)^{\text {reg }}\right) \times \mathfrak{X}_{\bar{\rho}^{p}}^{\square}\right) \subset \mathfrak{X}_{\bar{\rho}, \mathcal{S}} \times_{\mathfrak{X}_{\bar{\rho}}^{\square}} X_{\text {tri }}^{\square}\left(\bar{\rho}_{S}\right) .
$$

Si l'on suppose la Conjecture 3.22, il est donc dans $\mathfrak{X}_{\bar{\rho}, \mathcal{S}} \times_{\mathfrak{X}_{\overline{\rho_{S}}}^{\square}} X_{\text {tri }}^{\square}\left(\bar{\rho}_{S}\right)^{\text {aut }}$. Par le Théorème 4.2 , on en déduit que le point $\left(\rho_{\pi}, \iota\left(\prod_{v \in S_{p}} \delta_{w_{v}\left(k_{v}\right)} \eta_{v}\right)\right)$ est dans $Y\left(U^{p}, \bar{\rho}\right)$. Par [11, Prop. 8.1] appliqué avec $\Sigma_{p}=S_{p}=\{v \mid p\}$ (loc. cit. suppose $F_{v}^{+}=\mathbb{Q}_{p}$, mais ce point est inutile dans la preuve ici aussi), on en déduit que $Y\left(U^{p}, \bar{\rho}\right)$ contient tous les points $\left(\rho_{\pi}, \iota\left(\prod_{v \in S_{p}} \delta_{w_{v}^{\prime}\left(k_{v}\right)} \eta_{v}\right)\right)$ pour tout $w_{v}^{\prime}=\left(w_{\tau}^{\prime}\right)_{\tau: F_{v}^{+} \hookrightarrow L}$ dans $S_{n}^{\operatorname{Hom}\left(F_{v}^{+}, L\right)}$ tel que $w_{\tau}^{\prime} \leq w_{\tau}$ pour tous $\tau$ et $v$ (pour

3. Attention, ce n'est pas la Définition 2.8 du présent article. 
l'ordre de Bruhat sur $S_{n}$ ). En particulier, lorsque $F_{v}^{+}=\mathbb{Q}_{p}$ pour tout $v \in S_{p}$, on voit que la Conjecture 3.22 implique que tous les points compagnons de $\left(\rho_{\pi}, \iota(\delta)\right)$ prévus par [11, Conj. 6.5] pour $\Sigma_{p}=S_{p}$ (aux changements de notations près) sont bien dans $Y\left(U^{p}, \bar{\rho}\right)$.

4.2. Un résultat de modularité infinitésimale. Soit $\mathbf{k} \in \prod_{v \in S_{p}}\left(\mathbb{Z}^{n}\right)^{\left[F_{v}^{+}: \mathbb{Q}_{p}\right]}$ un poids strictement dominant. Fixons $\pi$ une représentation automorphe de $G$ vérifiant les conditions suivantes :

(i) $\pi^{U^{p}} \neq 0$;

(ii) pour $v \in S_{p}, \rho_{\pi, \tilde{v}}:=\left.\rho_{\pi}\right|_{\mathcal{G}_{\tilde{v}}}$ est cristalline de poids $\mathbf{k}_{v}$ et admet un raffinement non critique;

(iii) pour $v \in S_{p}$, les valeurs propres du Frobenius cristallin de $\rho_{\pi, \tilde{v}}$ sont deux-à-deux distinctes ;

(iv) $\bar{\rho}_{\pi}$ est isomorphe à $\bar{\rho}$.

Rappelons que l'on note $\bar{R}_{\infty}^{\mathrm{k}}$ l'image de $R_{\infty}$ dans l'anneau des endomorphismes $S_{\infty^{-}}$ linéaires de $\operatorname{Hom}_{K_{p}}\left(\sigma_{\mathbf{k}}, \Pi_{\infty}\right)$. D'après [15, Lem. 4.17], le $R_{\infty}$-module $\operatorname{Hom}_{K_{p}}\left(\sigma_{\mathbf{k}}, \Pi_{\infty}\right)^{\prime}$ est un $\bar{R}_{\infty}^{\mathrm{k}}[1 / p]$-module projectif de type fini. Comme par ailleurs $\operatorname{Hom}_{K_{p}}\left(\sigma_{\mathbf{k}}, \Pi_{\infty}\right)^{\prime}$ est un $S_{\infty}[1 / p]$-module projectif de type fini, l'anneau $\bar{R}_{\infty}^{\mathbf{k}}[1 / p]$ est une $S_{\infty}[1 / p]$-algèbre finie et plate.

La représentation $\rho_{\pi}$ correspond alors à un point de $\mathfrak{X}_{\bar{\rho}, \mathcal{S}}$. Notons $y$ son image dans $\operatorname{Spf}\left(R_{\infty}\right)^{\text {rig }}$ ainsi que $\left(y_{p}, y^{p}, y_{\infty}\right)$ ses coordonnées dans la décomposition

$$
\operatorname{Spf}\left(R_{\infty}\right)^{\text {rig }} \simeq \mathfrak{X}_{\bar{\rho}}^{\square, \mathbf{k}} \times \mathfrak{X}_{\bar{\rho}^{p}}^{\square} \times \mathbb{U}^{g} .
$$

D'après [13, Thm. 1.2], la représentation $\pi$ est tempérée en toute place de $F^{+}$. En particulier, on déduit de [3, Lem. 1.3.2.(1)] que le point $y^{p}$ est un point lisse de $\mathfrak{X}_{\bar{\rho}^{p}}^{\square}$, il appartient donc à une unique composante irréductible de cet espace. Fixons $\chi=\bigotimes_{v \in S_{p}} \chi_{v}$ un caractère lisse non ramifié de $T_{p}$ tel que $\delta_{v}:=\delta_{\mathbf{k}_{v}} \chi_{v}$ est un paramètre non critique de $\rho_{\pi, \tilde{v}}$ pour tout $v \in S_{p}$, et donc, en posant $\delta=\bigotimes_{v \in S_{p}} \delta_{v}$, que $\left(\rho_{\pi}, \delta\right) \in Y\left(U^{p}, \bar{\rho}\right)^{\mathrm{reg}}$. Si $F_{v}^{+}=\mathbb{Q}_{p}$, on déduit de [4, Prop. 6.4.8] et de la remarque 4.5 qu'il existe toujours un tel caractère et la partie non critique de l'hypothèse (ii) ci-dessus est superflue. En particulier, pour tout $v \in S_{p}$, on a $\left(\rho_{\pi, \tilde{v}}, \delta_{v}\right) \in U_{\mathrm{tri}}^{\square}\left(\bar{\rho}_{\pi, \tilde{v}}\right)^{\mathrm{reg}}$.

Lemme 4.6. Le schéma $\operatorname{Spec}\left(\bar{R}_{\infty}^{\mathbf{k}}[1 / p]\right)$ est fini étale au-dessus d'un ouvert de Zariski de $\operatorname{Spec}\left(S_{\infty}[1 / p]\right)$. De plus, le point y provenant de $\pi$ appartient à cet ouvert.

Démonstration. Comme $\bar{R}_{\infty}^{\mathrm{k}}[1 / p]$ est une $S_{\infty}[1 / p]$-algèbre finie et plate et que $S_{\infty}[1 / p]$ est intègre, il suffit de montrer qu'il existe un point fermé de $\operatorname{Spec}\left(\bar{R}_{\infty}^{\mathrm{k}}[1 / p]\right)$ où le morphisme $\operatorname{Spec}\left(\bar{R}_{\infty}^{\mathbf{k}}[1 / p]\right) \rightarrow \operatorname{Spec}\left(S_{\infty}[1 / p]\right)$ est étale. Pour prouver les deux assertions simultanément, il suffit de prouver que tous les points fermés de $\operatorname{Spec}\left(\bar{R}_{\infty}^{\mathrm{k}}[1 / p]\right)$ au-dessus de l'idéal d'augmentation $\mathfrak{a}$ de $S_{\infty}[1 / p]$ sont étales, autrement dit que l'anneau $\bar{R}_{\infty}^{\mathrm{k}}[1 / p] / \mathfrak{a}$ est réduit. Or, $\operatorname{Hom}_{K_{p}}\left(\sigma_{\mathbf{k}}, \Pi_{\infty}\right)^{\prime} / \mathfrak{a} \simeq \operatorname{Hom}_{K_{p}}\left(\sigma_{\mathbf{k}}, \Pi_{\infty}[\mathfrak{a}]\right)$ est l'espace de formes automorphes $S\left(U^{p} K_{p}, \sigma\right)_{\bar{\rho}}$. Comme $\operatorname{Hom}_{K_{p}}\left(\sigma_{\mathbf{k}}, \Pi_{\infty}\right)^{\prime}$ est un $\bar{R}_{\infty}^{\mathbf{k}}[1 / p]$-module localement libre de type fini et fidèle, $\bar{R}_{\infty}^{\mathbf{k}}[1 / p] / \mathfrak{a}$ s'identifie à la sous- $L$-algèbre des endomorphismes $L$-linéaires de $S\left(U^{p} K_{p}, \sigma\right)_{\bar{\rho}}$ engendrée par les opérateurs de Hecke hors de $S$ qui est bien semi-simple.

On note $X_{\text {tri }}^{\square}\left(\bar{\rho}_{p}\right)_{\mathbf{k}}$ la fibre $\omega^{-1}\left(\delta_{\mathbf{k}}\right)$ et $U_{\text {tri }}^{\square}\left(\bar{\rho}_{p}\right)_{\mathbf{k}}^{\text {reg }}$ son intersection avec $U_{\text {tri }}^{\square}\left(\bar{\rho}_{p}\right)^{\text {reg }}$. De même, on note $X_{p}(\bar{\rho})^{\text {reg }}$ et $X_{p}(\bar{\rho})_{\mathbf{k}}^{\text {reg }}$ les intersections respectives de $X_{p}(\bar{\rho})$ avec l'espace $\iota\left(U_{\text {tri }}^{\square}(\bar{\rho})^{\text {reg }}\right) \times \mathfrak{X}_{\bar{\rho}^{p}}^{\square, \text { reg }} \times \mathbb{U}^{g}$ et $\iota\left(U_{\text {tri }}^{\square}(\bar{\rho})_{\mathbf{k}}^{\text {reg }}\right) \times \mathfrak{X}_{\bar{\rho}^{p}}^{\square, \text { reg }} \times \mathbb{U}^{g}$. 
Proposition 4.7. (i) L'ensemble $U_{\mathbf{k}}^{\text {ét }} \subset X_{p}(\bar{\rho})_{\mathbf{k}}^{\mathrm{reg}}$ des points de $X_{p}(\bar{\rho})_{\mathbf{k}}^{\mathrm{reg}}$ où l'application $\omega_{X}$ de $X_{p}(\bar{\rho})$ dans $\mathcal{W}_{\infty}$ est étale forme un ouvert dense de Zariski de $X_{p}(\bar{\rho})_{\mathbf{k}}^{\mathrm{reg}}$.

(ii) Si $z \in Y\left(U^{p}, \bar{\rho}\right)$ est le point associé à $(\pi, \iota(\delta))$, alors l'image de $z$ dans $X_{p}(\bar{\rho})$ appartient $\grave{a} U_{\mathbf{k}}^{\text {ét }}$.

Démonstration. Par changement de base au dessus de l'inclusion canonique d'une fibre $\left\{\iota\left(\delta_{\mathbf{k}}\right)\right\} \times \operatorname{Spf}\left(S_{\infty}\right)^{\text {rig }} \hookrightarrow \mathcal{W}_{\infty}$, on sait qu'en un point de $X_{p}(\bar{\rho})_{\mathbf{k}}^{\text {reg }}$ où l'application $\omega_{X}$ est étale, l'application $X_{p}(\bar{\rho})_{\mathbf{k}}^{\text {reg }} \rightarrow \operatorname{Spf}\left(S_{\infty}\right)^{\text {rig }}$ (déduite par changement de base) est a fortiori étale. Comme l'action de $S_{\infty}$ sur $\Pi_{\infty}$ se factorise par $R_{\infty}$, on a un diagramme commutatif

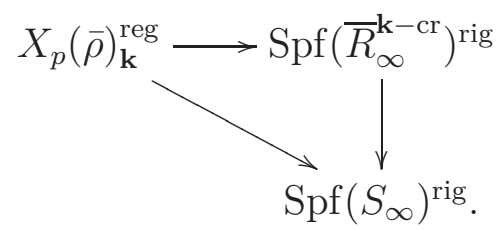

En effet, si $v \in S_{p}$, d'après [35, Prop. 2.6], le relèvement $\rho_{\tilde{v}}$ de $\bar{\rho}_{\tilde{v}}$ correspondant à un point de $X_{p}(\bar{\rho})_{\mathbf{k}}^{\mathrm{reg}}$ est potentiellement semi-stable. De plus comme $\rho_{\tilde{v}}$ est trianguline de paramètre $\delta_{v} \in \mathcal{T}_{\text {reg }}^{n}$, le quotient de deux valeurs propres distinctes de son Frobenius cristallin n'appartient pas à $\left\{1, q_{v}\right\}$, donc $\rho_{\tilde{v}}$ est nécessairement de monodromie nulle, c'est-à-dire cristalline.

En utilisant le Théorème 3.20 ainsi que (le raisonnement de) [35, Prop. 4.8], on montre que la flèche horizontale supérieure est étale. En particulier, l'ensemble recherché est contenu dans l'ensemble des points de $\operatorname{Spf}\left(\bar{R}_{\infty}^{\mathrm{k}-\mathrm{cr}}\right)^{\text {rig }}$ où la flèche verticale en (4.1) est étale. Remarquons que ce dernier ensemble coïncide avec l'ensemble des points fermés de $\operatorname{Spec}\left(\bar{R}_{\infty}^{\mathrm{k}-\mathrm{cr}}[1 / p]\right)$ où $\operatorname{Spec}\left(\bar{R}_{\infty}^{\mathrm{k}-\mathrm{cr}}[1 / p]\right)$ est étale au-dessus de $\operatorname{Spec}\left(S_{\infty}[1 / p]\right)$. Définissons alors $U_{\mathbf{k}}^{\text {ét }} \subset X_{p}(\bar{\rho})_{\mathbf{k}}^{\text {reg }}$ comme l'image réciproque de l'ensemble des points fermés de $\operatorname{Spec}\left(\bar{R}_{\infty}^{\mathbf{k}-\mathrm{cr}}[1 / p]\right)$ pour lesquels l'application $\operatorname{Spec}\left(\bar{R}_{\infty}^{\mathrm{k}-\mathrm{cr}}[1 / p]\right) \rightarrow \operatorname{Spec}\left(S_{\infty}[1 / p]\right)$ est étale. Le Lemme 4.6 (avec l'intégrité de $S_{\infty}[1 / p]$ ) montre alors que pour prouver (i), il suffit de prouver qu'aux points de $U_{\mathbf{k}}^{\text {ét }}$, l'application $\omega_{X}$ de $X_{p}(\bar{\rho})$ dans $\mathcal{W}_{\infty}$ est étale.

Soit $x \in U_{\mathbf{k}}^{\text {ét }}$. Considérons le diagramme suivant

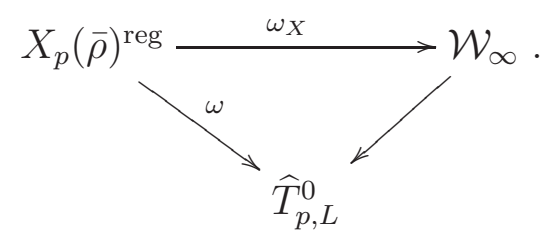

Les deux flèches diagonales sont lisses. Celle de droite car il s'agit d'une projection de fibre lisse. Celle de gauche car il s'agit d'une projection de fibre lisse, composée avec l'application poids $X_{\text {tri }}^{\square}\left(\bar{\rho}_{p}\right) \rightarrow \widehat{T}_{p, L}^{0}$ qui est lisse au point $y_{p}$ d'après le (iii) du Théorème 2.6 et le fait que $X_{p}(\bar{\rho})^{\text {reg }}$ est un ouvert de $\iota\left(U_{\text {tri }}^{\square}\left(\bar{\rho}_{p}\right)^{\text {reg }}\right) \times \mathfrak{X}_{\bar{\rho}^{p}}^{\square} \times \mathbb{U}^{g}$ (ce qui se déduit des Théorèmes 2.6 (ii) et 3.20). On déduit alors du Lemme 5.7 de l'appendice que l'application horizontale est lisse si et seulement si l'application obtenue par changement de base entre les fibres au-dessus de $\omega(y)$ est lisse. Or, par changement de base, $\omega_{X}$ devient la flèche diagonale du diagramme (4.1) et par définition de $U_{\mathrm{k}}^{\text {ét }}$ l'application horizontale est lisse après changement de base, ce qui nous permet de conclure.

Le point (ii) est alors une application immédiate de (i) et du fait que le point classique $z \in Y\left(U^{p}, \bar{\rho}\right) \subset \iota\left(X_{\text {tri }}^{\square}\left(\bar{\rho}_{p}\right)\right) \times \mathfrak{X}_{\bar{\rho}^{p}}^{\square} \times \mathbb{U}^{g}$ est en fait dans $\iota\left(U_{\text {tri }}^{\square}\left(\bar{\rho}_{p}\right)^{\text {reg }}\right) \times \mathfrak{X}_{\bar{\rho}^{p}}^{\square \text { reg }} \times \mathbb{U}^{g}$ (cf. la discussion précédant le Lemme 4.6). 
On peut également s'intéresser à la structure « schématique » de l'espace $\mathfrak{X}_{\bar{\rho}, \mathcal{S}} \times_{\mathfrak{X}_{\bar{\rho}}^{\square}} X_{\text {tri }}^{\square}\left(\bar{\rho}_{S}\right)$. Dans cette direction, on a le résultat suivant.

Théorème 4.8. Les espaces analytiques rigides $\mathfrak{X}_{\bar{\rho}, \mathcal{S}} \times_{\mathfrak{X}_{\bar{\rho}_{S}}^{\square}} \iota\left(X_{\mathrm{tri}}^{\square}\left(\bar{\rho}_{S}\right)^{\text {aut,reg }}\right)$ et $Y\left(U^{p}, \bar{\rho}\right)^{\text {reg }}$ sont isomorphes.

Démonstration. Comme ces deux espaces rigides analytiques ont le même espaces sousjacent, il suffit de prouver qu'ils sont tous les deux réduits. L'espace $Y\left(U^{p}, \bar{\rho}\right)$ est le sous-espace fermé de $\operatorname{Spf}\left(R_{\infty}\right)^{\text {rig }} \times \widehat{T}_{p, L}$ défini par l'idéal annulateur $\mathcal{I}_{U^{p}}$ du faisceau cohérent $\mathcal{M}_{U^{p}}$ défini par $J_{B_{p}}\left(\widehat{S}\left(U^{p}, L\right)_{\bar{\rho}}^{\text {an }}\right)$. Comme ce faisceau est annulé par l'idéal a, le faisceau d'idéaux $\mathcal{I}_{U^{p}}$ contient le sous-faisceau d'idéaux engendré par a et par l'idéal annulateur de $\mathcal{M}_{\infty}$. Ceci implique que l'inclusion $Y\left(U^{p}, \bar{\rho}\right) \subset X(\bar{\rho})_{\text {fs }}$ est en fait un morphisme d'espaces analytiques rigides. D'après le Théorème 4.2 les espaces $Y\left(U^{p}, \bar{\rho}\right)^{\text {reg }}$ et $\mathfrak{X}_{\bar{\rho}, \mathcal{S}} \times_{\mathfrak{X}_{\bar{\rho}_{S}}^{\square}} \iota\left(X_{\text {tri }}^{\square}\left(\bar{\rho}_{S}\right)^{\text {aut,reg }}\right)$ ont même espace sous-jacent. Il suffit donc de prouver que l'espace

$$
\mathfrak{X}_{\bar{\rho}, \mathcal{S}} \times_{\mathfrak{X}_{\bar{\rho}}^{\square}} \iota\left(X_{\text {tri }}^{\square}\left(\bar{\rho}_{S}\right)^{\text {aut,reg }}\right)=\operatorname{Sp}(L) \times_{\operatorname{Spf}\left(S_{\infty}\right)^{\text {rig }} X_{p}(\bar{\rho})^{\text {reg }}}
$$

est réduit. Il résulte du Corollaire 3.11 et de $[27, \S 3.2]$ que les espaces $X_{p}(\bar{\rho})$ et $Y\left(U^{p}, \bar{\rho}\right)$ sont tous deux équidimensionnels de dimensions respectives $g+\left[F^{+}: \mathbb{Q}\right] \frac{n(n+1)}{2}+|S| n^{2}$ et $\left[F^{+}: \mathbb{Q}\right] n$. Ainsi $\operatorname{dim}\left(X_{p}(\bar{\rho})\right)-\operatorname{dim}\left(Y\left(U^{p}, \bar{\rho}\right)\right)$ est égal à $q$ où $q$ est aussi le cardinal d'une suite de générateurs $y_{1}, \ldots, y_{q}$ de $\mathfrak{a}$. Par ailleurs, comme $X_{p}(\bar{\rho})$ est régulier aux points de $X_{p}(\bar{\rho})^{\mathrm{reg}}$, il est en particulier de Cohen-Macaulay en ces points, on déduit donc de [31, Cor. 16.5.6] et de l'égalité ci-dessus que la suite $y_{1}, \ldots, y_{q}$ est une suite régulière sur $X_{p}\left(\bar{\rho}_{p}\right)^{\text {reg }}$ et que $\operatorname{Sp}(L) \times_{\operatorname{Spf}\left(S_{\infty}\right)^{\text {rig }}} X_{p}(\bar{\rho})^{\text {reg }}$ est de Cohen-Macaulay. Ceci implique en particulier que cet espace analytique rigide est sans composante immergée (cf. [31, Prop. 16.5.4]). Pour conclure qu'il est réduit, il suffit donc de prouver que chaque composante irréductible contient un point réduit. C'est une conséquence de la Proposition 4.7 et du fait que pour toute composante irréductible $X$ de $X_{p}(\bar{\rho}), X \cap X_{p}(\bar{\rho})^{\text {reg }}$ contient un point de poids algébrique strictement dominant d'après le Corollaire 3.12.

Remarque 4.9. Le point (ii) de la Proposition 4.7 ainsi que le Théorème 4.8 donnent une autre preuve du Théorème 4.10 de [18].

Si $v \in S_{p}$, désignons par $\mathcal{R}_{v}$ le raffinement de $\rho_{\pi, \tilde{v}}$ associé au caractère $\chi_{v}=\left.\chi\right|_{T_{v}}$ de $T_{v}$ et $\mathcal{R}=\left(\mathcal{R}_{v}\right)_{v \in S_{p}}$. Soit $X_{\rho_{\pi, \tilde{v}}}$ le foncteur des déformations de $\rho_{\pi, \tilde{v}}$ et $X_{\rho_{\pi, \tilde{v}}, \mathcal{R}_{v}}$ le foncteur des déformations de la paire $\left(\rho_{\pi, \tilde{v}}, \mathcal{R}_{v}\right)$ tel que défini dans $[4, \S 2.5]$. D'après [4, Prop. 2.5.8], la transformation naturelle $X_{\rho_{\pi, \tilde{v}}, \mathcal{R}_{v}} \rightarrow X_{\rho_{\pi, \tilde{v}}}$ est relativement représentable, et on note $R_{\rho_{\pi, \tilde{v}}, \mathcal{R}_{v}}^{\square}$ l'algèbre locale complète pro-représentant le foncteur $X_{\rho_{\pi, \tilde{v}}, \mathcal{R}_{v}} \times_{X_{\rho_{\pi, \tilde{v}}}} \operatorname{Spf}\left(R_{\rho_{\pi, \tilde{v}}}^{\bar{\square}}\right)$.

Notons alors $R_{\rho_{\pi}, S, \mathcal{R}}$ l'anneau pro-représentant le foncteur des déformations du couple $\left(\rho_{\pi}, \mathcal{R}\right)$ défini comme en $[4, \S 2.6]$. Plus précisément il s'agit de l'anneau $R_{\rho_{\pi}, S} \widehat{\otimes}_{R_{\rho_{\pi, p}}^{\square}} R_{\rho_{\pi, p}, \mathcal{R}}^{\square}$ avec $R_{\rho_{\pi, p}, \mathcal{R}}^{\square}=\widehat{\bigotimes}_{v \in S_{p}} R_{\rho_{\pi, \tilde{v}}, \mathcal{R}_{v}}^{\square}$, où $\rho_{\pi, S}$ désigne le séparé complété du localisé de $R_{\bar{\rho}_{\pi}}[1 / p]$ en l'idéal maximal associé à $\rho_{\pi}$.

Lemme 4.10. L'anneau $R_{\rho_{\pi}, S}$ est aussi le séparé complété du localisé de $R_{\bar{\rho}_{\pi}, \mathcal{S}}[1 / p]$ en l'idéal associé à $\rho_{\pi}$.

Démonstration. Comme $R_{\bar{\rho}_{\pi}, \mathcal{S}}=R_{\bar{\rho}_{\pi}, S} \otimes_{R_{\bar{\rho}_{\pi, S}}} R_{\bar{\rho}_{\pi, S}}^{\bar{C}}$, il suffit de vérifier que pour $v \in S$, le séparé complété du localisé de $R_{\bar{\rho}_{\pi, \tilde{v}}}$ au point correspondant à $\rho_{\pi, \tilde{v}}$ est réduit. Pour $v \in S \backslash S_{p}$, c'est une conséquence de la discussion précédant le Lemme 4.7. Quant au 
cas $v \in S_{p}$, il suffit de vérifier que le problème de déformations de $\rho_{\pi, \tilde{v}}$ est non obstrué, autrement dit que $H_{\text {cont }}^{2}\left(\mathcal{G}_{F_{\tilde{v}}}, \operatorname{ad}\left(\rho_{\pi, \tilde{v}}\right)\right)=0$. Par dualité de Tate, il suffit de prouver que $H^{0}\left(\mathcal{G}_{F_{\tilde{v}}}, \operatorname{ad}\left(\rho_{\pi, \tilde{v}}(1)\right)\right)=0$. Or il suffit de vérifier ceci au niveau des $(\varphi, \Gamma)$-modules sur $\mathcal{R}_{L, F_{\tilde{v}}}$, c'est alors une conséquence du fait que $\rho_{\pi, \tilde{v}}$ est trianguline de paramètre dans $\mathcal{T}_{\text {reg }}^{n}$.

Corollaire 4.11. Si $p>2 n+1$, la Conjecture 7.6.12 $(R=T)$ de [4] est vraie au point $y=\left(\rho_{\pi}, \iota(\delta)\right)$ de $Y\left(U^{p}, \bar{\rho}\right)$. Plus précisément, on a un isomorphisme d'anneaux locaux complets

$$
R_{\rho_{\pi}, S, \mathcal{R}} \stackrel{\sim}{\rightarrow} \widehat{\mathcal{O}}_{Y\left(U^{p}, \bar{\rho}\right), y} .
$$

Démonstration. La condition sur les valeurs de $\chi_{i}\left(\varpi_{v}\right)$ et le fait que $\chi$ corresponde à un raffinement non critique de $\pi$, implique que l'image de $z$ dans $X_{\text {tri }}^{\square}\left(\bar{\rho}_{p}\right)$ appartient à l'ouvert lisse $U_{\text {tri }}^{\square}\left(\bar{\rho}_{p}\right)^{\text {reg }}$ et se situe donc sur une unique composante irréductible de $X_{\text {tri }}^{\square}\left(\bar{\rho}_{p}\right)$. Ceci implique que l'anneau local complété de $Y\left(U^{p}, \bar{\rho}\right)$ en $z$ est isomorphe à l'anneau local complété de $X_{\mathrm{fs}}(\bar{\rho})$ en $z$. Or cet anneau local complété est exactement $R_{S, \rho_{\pi}} \widehat{\otimes}_{R_{\rho_{\pi, p}}} R_{\rho_{\pi, p}, \mathcal{R}}^{\square}$ qui est canoniquement isomorphe à $R_{S, \rho_{\pi, p}, \mathcal{R}}$.

Corollaire 4.12. Si A est une L-algèbre locale de dimension finie, toute déformation de $\rho_{\pi}$ sur $A$ vérifiant $\bar{\rho}_{\pi}^{\vee} \circ c \simeq \rho_{\pi}(n-1)$ et cristalline en toute place divisant $p$ est triviale.

Démonstration. D'après le théorème 4.10 de [18] (ou la Remarque 4.9), l'application poids est étale au point $z=(\pi, \chi)$ de $Y\left(U^{p}, \bar{\rho}\right)$. Le résultat est alors une conséquence du corollaire 7.6.14 de [4] et du corollaire 4.11.

Le même raisonnement que [41, Thm. 8.2] montre alors le résultat suivant sur la géométrie de l'espace $\operatorname{Spec}\left(R_{S}\left(\bar{\rho}_{\pi}\right)[1 / p]\right)$ au point $\rho_{\pi}$.

Corollaire 4.13. L'espace $\operatorname{Spec}\left(R_{S}\left(\bar{\rho}_{\pi}\right)[1 / p]\right)$ est lisse et de dimension $\frac{n(n+1)}{2}\left[F^{+}: \mathbb{Q}\right]$ au point $\rho_{\pi}$.

Démonstration. D’après le Lemme 4.10, on a un isomorphisme

$$
R_{\rho_{\pi}, S} \otimes_{R_{\rho_{\pi}, p}^{\square}} R_{\rho_{\pi}, p}^{\square, \mathbf{k}-\mathrm{cr}} \simeq R_{\rho_{\pi}, \mathcal{S}} \otimes_{R_{\rho_{\pi}, p}^{\square}} R_{\rho_{\pi}, p}^{\square, \mathrm{k}-\mathrm{cr}} .
$$

En raisonnant comme dans [4, Prop. 7.6.4], on peut alors déduire du Corollaire 4.11 et de [18, Thm. 4.10] (ou de la Remarque 4.9) que

$$
\operatorname{Ker}\left(\operatorname{Hom}_{L-\operatorname{alg}}\left(R_{\rho_{\pi}, S}, L[X] /\left(X^{2}\right)\right) \rightarrow \operatorname{Hom}_{L-\operatorname{alg}}\left(R_{\rho_{\pi, p}}^{\square, \mathbf{k}-\text { cris }}, L[X] /\left(X^{2}\right)\right)\right)=\{0\} .
$$

En raisonnant exactement comme dans la preuve de [41, Thm. 8.2], on en déduit que $\operatorname{Hom}_{L-\operatorname{alg}}\left(R_{\rho_{\pi}, S}, L[X] /\left(X^{2}\right)\right)$, autrement dit l'espace tangent $\operatorname{de} \operatorname{Spec}\left(R_{\bar{\rho}_{\pi}, S}\right)$ au point $\rho_{\pi}$ est de dimension $\leq \frac{n(n+1)}{2}\left[F^{+}: \mathbb{Q}\right]$. Comme par ailleurs, d'après [21, Cor. 2.2.12], on a $\operatorname{dim}\left(R_{\bar{\rho}_{\pi}, S}\right) \geq 1+\frac{n(n+1)}{2}\left[F^{+}: \mathbb{Q}\right]$, on en déduit le résultat.

Remarque 4.14. Sous des hypothèses plus générales, essentiellement en ne supposant pas $\pi$ non ramifiée en $p$, les deux corollaires précédents ont été prouvés de façon indépendante par Allen dans [2]. 


\section{Appendice}

5.1. Un peu d'analyse fonctionnelle non archimédienne. Soit $H$ un pro- $p$-groupe uniforme. Fixons $A$ une $\mathcal{O}_{L}$-algèbre quotient sans $p$-torsion de l'algèbre de groupe complétée $\mathcal{O}_{L} \llbracket H \rrbracket$ (l'algèbre $A$ n'est donc pas nécessairement commutative). Si $\mathfrak{m}_{A}$ désigne l'idéal maximal de $A$, on note, pour $n \geq 1, A_{n}^{\circ}$ le complété $\mathfrak{m}_{A}$-adique de la $\mathcal{O}_{L}$-algèbre $A\left[\frac{\mathfrak{m}_{A}^{n}}{\varpi}\right] \subset A\left[\frac{1}{p}\right]$ et on pose $A_{n}=A_{n}^{\circ}\left[\frac{1}{p}\right]$. On définit alors, comme dans le cas commutatif $A^{\text {rig }}=\lim _{n} A_{n}$. Notons $D(H)$ l'algèbre des distributions de $H$ et $I$ le noyau de $\mathcal{O}_{L} \llbracket H \rrbracket \rightarrow A$.

Lemme 5.1. La $\mathcal{O}_{L}$-algèbre $A^{\text {rig }}$ est une algèbre de Fréchet-Stein, qui est isomorphe à $D(H) / I D(H)$.

Démonstration. D'après le théorème 5.4 de [55], l'algèbre $D(H)$ est de Fréchet-Stein, munie d'une famille de normes $\left(q_{n}\right)_{n>1}$ définie comme suit. On choisit $\left(h_{1}, \cdots, h_{d}\right)$ une base ordonnée de $H$, et pour tout $\alpha \in \overline{\mathbb{N}}^{d}$, on pose $b^{\alpha}=b_{1}^{\alpha_{1}} \cdots b_{d}^{\alpha_{d}} \in \mathcal{O}_{L}[H]$ où $b_{i}=h_{i}-1$. On pose alors, pour $x=\sum_{\alpha} x_{\alpha} b^{\alpha}, q_{n}(x)=\sup _{\alpha}\left(\left|a_{\alpha}\right| p^{-|\alpha| / n}\right)$. La boule unité de $\mathcal{O}_{L} \llbracket H \rrbracket$ pour la norme $q_{n}$ est donc exactement $\mathcal{O}_{L} \llbracket H \rrbracket_{n}^{\circ}$. Ceci implique que $A_{n}^{\circ}$ est la boule unité de $A$ muni de la semi-norme quotient induite par $q_{n}$. La proposition 3.7 de [55] implique alors que $A^{\text {rig }}$

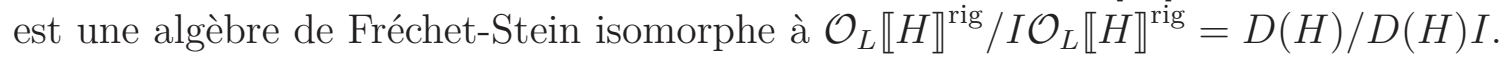

Lemme 5.2. Supposons que $\varphi: A \rightarrow B$ soit un morphisme local fini entre deux algèbres locales noethériennes complète sans p-torsion. Supposons que $B \varphi\left(\mathfrak{m}_{A}\right)=\varphi\left(\mathfrak{m}_{A}\right) B$. Alors le morphisme $A^{\mathrm{rig}} \otimes_{A} B \rightarrow B^{\mathrm{rig}}$ est un isomorphisme.

Démonstration. Remarquons que $A^{\text {rig }} \otimes_{A} B$ est un $A^{\text {rig }}$-module coadmissible, on a donc d'après [55, Cor. 3.1], un isomorphisme $A^{\text {rig }} \otimes_{A} B \simeq \lim _{n} A_{n} \otimes_{A} B$. En raisonnant comme dans la preuve du lemme 7.2.2 de [38], on obtient l'isomorphisme

$$
B^{\mathrm{rig}} \simeq \underset{\lim _{n}}{\lim }\left(A_{n} \widehat{\otimes}_{A} B\right) \text {. }
$$

5.2. Vecteurs localement analytiques. Les résultats présentés ici sont essentiellement issus de $[26, \S 4]$. Nous en donnons ici une formulation un peu plus adaptée à nos besoins.

Soit $K$ une extension finie de $\mathbb{Q}_{p}$ et $G$ le groupe des $K$-points d'un groupe réductif quasi-déployé défini sur $K$. Fixons $B$ (le groupe des $K$-points d') un sous-groupe de Borel de $G$. Soit $N$ le radical unipotent de $B$ et $M$ un sous-groupe de Levi de $B$ de sorte que $B=M N$. On note $\bar{B}$ le sous-groupe de Borel opposé à $B$ relativement à $M$ et $\bar{N}$ son radical unipotent. Soit $M^{0}$ le sous-groupe compact maximal de $M$, il s'agit d'un tore.

Fixons $q \geq 0$ et posons $\widetilde{G}=G \times \mathbb{Z}_{p}^{q}$. Si $H$ est un sous-groupe de $G$, on pose $\widetilde{H}=H \times \mathbb{Z}_{p}^{q}$. On note $\mathcal{W}$ l'espace des caractères continus de $M^{0} \times \mathbb{Z}_{p}^{q}$. On choisit $H$ un sous-pro- $p$ groupe ouvert et uniforme de $G$, tel que la multiplication

$$
(\bar{N} \cap H) \times(M \cap H) \times(N \cap H) \rightarrow H
$$

soit un homéomorphisme. On pose $N_{0}=N \cap H$, ainsi $N_{0}$ est stable sous l'action de $M^{0}$ par conjugaison. Soit $\Pi$ une représentation unitaire admissible de $\widetilde{G}$ sur un $L$-espace de Banach. On suppose par ailleurs que $\left.\Pi\right|_{\widetilde{H}}$ est isomorphe à $\mathcal{C}(\widetilde{H}, L)^{m}$ pour un certain 
$m \geq 0$. On définit une action, dite de Hecke, du monoïde $M^{+}=\left\{z \in M \mid z N_{0} z^{-1} \subset N_{0}\right\}$ sur $\Pi^{N_{0}}$ par la formule

$$
z \cdot v=\sum_{n \in N_{0} / z N_{0} z^{-1}} n z v
$$

De plus on fixe un élément $z \in M^{+}$tel que $\bigcap_{n \geq 0} z^{n} N_{0} z^{-n}=\{1\}$.

Proposition 5.3. Il existe un recouvrement admissible de $\mathcal{W}$ par des ouverts affinoïdes $U_{1} \subset U_{2} \subset \cdots \subset U_{h} \subset \cdots$ et, pour tout $h \geq 1$,

- un $A_{h}=\mathcal{O}_{\mathcal{W}}\left(U_{h}\right)$-module de Banach $V_{h}$ vérifiant la condition $(P r)$ de [12];

- un endomorphisme $A_{h}$-compact, noté $z_{h}$, du $A_{h}$-module $V_{h}$ (la notion d'endomorphisme $A_{h}$-compact d'un espace de type (Pr) est définie dans $\left.[12, \S 2]\right)$;

- des applications $A_{h}$-linéaires continues

$$
\alpha_{h}: V_{h} \longrightarrow V_{h+1} \widehat{\otimes}_{A_{h+1}} A_{h} \text { et } \beta_{h}: V_{h+1} \widehat{\otimes}_{A_{h+1}} A_{h} \rightarrow V_{h}
$$

telles que $\beta_{h} \circ \alpha_{h}=z_{h}$ et $\alpha_{h} \circ \beta_{h}=z_{h+1} \otimes 1_{A_{h}}$;

- un isomorphisme topologique $\mathcal{O}(\mathcal{W})$-linéaire $\left(\left(\Pi^{\text {an }}\right)^{N_{0}}\right)^{\prime} \simeq \lim _{h} V_{h}$ commutant à l'action de $z$ à gauche et de $\left(z_{h}\right)_{h \geq 1}$ à droite.

On peut résumer toutes ces conditions dans le diagramme commutatif suivant

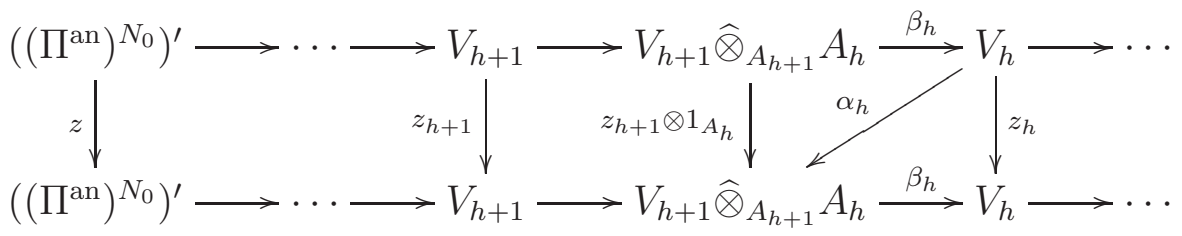

Démonstration. Pour $\frac{1}{p}<r<1$, on note $D_{r}(H, L)$ et $D_{<r}(H, L)$ les algèbres de Banach définies dans $[55, \S 3]$. Si $h \geq 1$, posons en suivant $[23, \S 0.3], r_{h}=\frac{1}{p^{h-1}(p-1)}$ et

$$
B_{h}=D_{p^{r}}(M \cap H, L) .
$$

On note $\Pi_{\widetilde{H}}^{(h)} \subset \Pi$ le sous-espace des vecteurs $r_{h}$-analytiques de $\Pi$ défini dans loc. cit. Il s'agit d'un $L$-espace de Banach, isomorphe à $\mathcal{C}^{(h)}(\widetilde{H}, L)^{m}$ comme représentation de $\widetilde{H}$, avec les notations de [23]. On vérifie facilement que l'action de $z$ sur $\Pi$ envoie $\Pi_{\widetilde{H}}^{(h)}$ dans $\Pi_{z \widetilde{H} z^{-1}}^{(h)}$. Par ailleurs, l'action de Hecke de $z$ préserve $\Pi^{N_{0}}$, on a en fait $z \cdot\left(\Pi_{\widetilde{H}}^{(h)}\right) \subset \Pi_{z \widetilde{H} z^{-1} N_{0}}^{(h)}$. En identifiant

$$
\left(\Pi_{\widetilde{H}}^{(h)}\right)^{N_{0}}=\mathcal{C}^{(h)}(\bar{N} \cap H, L)^{m} \widehat{\otimes}_{L} \mathcal{C}^{(h)}(\widetilde{M} \cap \widetilde{H}, L),
$$

ceci prouve que si $v=\sum_{n \geq 0} a_{n} \otimes b_{n} \in \mathcal{C}^{(h)}(\bar{N} \cap H, L)^{m} \widehat{\otimes}_{L} \mathcal{C}^{(h)}(\widetilde{M} \cap \widetilde{H}, L)$, alors on peut écrire $z(v)=\sum_{n \geq 0} a_{n}^{\prime} \otimes b_{n}^{\prime}$, où $b_{n}^{\prime} \in \mathcal{C}^{(h)}(\widetilde{M} \cap \widetilde{H}, L)$ et $a_{n}^{\prime}$ est la restriction à $\bar{N} \cap H$ d'un élément de $\overline{\mathcal{C}}^{(h)}\left(z(\bar{N} \cap H) z^{-1}, L\right)^{m}$. D'après [23, Thm. 0.5.(ii)] et la condition $\bigcap_{n \geq 0} z^{n} N_{0} z^{-n}=\{1\}$, l'application de restriction $\mathcal{C}^{(h)}\left(z(\bar{N} \cap H) z^{-1}, L\right) \rightarrow \mathcal{C}^{(h)}(\bar{N} \cap H, L)$ se factorise à travers $\mathcal{C}^{(h-1)}(\bar{N} \cap H, L)$, ce qui prouve au final que

$$
z\left(\left(\mathcal{C}^{(h)}(\widetilde{H}, L)^{m}\right)^{N_{0}}\right) \subset \mathcal{C}^{(h-1)}(\bar{N} \cap H, L)^{m} \widehat{\otimes}_{L} \mathcal{C}^{(h)}(\widetilde{M} \cap \widetilde{H}, L) .
$$

Comme le dual de $\mathcal{C}^{(h)}(\widetilde{M} \cap \widetilde{H}, L)$ est l'algèbre $D_{<p^{r} h}(\widetilde{H} \cap \widetilde{M}, L)$ et que pour tout $h$ la flèche d'inclusion $B_{h+1} \rightarrow B_{h}$ se factorise par $D_{<p^{r} h+1}(\widetilde{H}, L)$, on peut poser $V_{h}=W_{h} \widehat{\otimes}_{L} B_{h}$, où $W_{h}=\left(\mathcal{C}^{(h)}(\bar{N} \cap H, L)^{m}\right)^{\prime}$ de telle sorte que l'on ait un isomorphisme topologique de $B_{h}$-modules

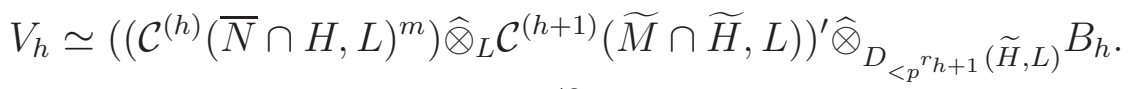


Ainsi $V_{h}$ est un $B_{h}$-module orthonormalisable. On définit alors $\alpha_{h}$ comme la flèche $\alpha_{h}^{\prime} \otimes 1_{B_{h}}$ où $\alpha_{h}^{\prime}$ est l'application duale de l'application

$$
\mathcal{C}^{(h+1)}(\bar{N} \cap H, L)^{m} \widehat{\otimes}_{L} \mathcal{C}^{(h+1)}(\widetilde{M} \cap \widetilde{H}, L) \hookrightarrow \mathcal{C}^{(h)}(\bar{N} \cap H, L)^{m} \widehat{\otimes}_{L} \mathcal{C}^{(h+1)}(\widetilde{M} \cap \widetilde{H}, L)
$$

induite par l'action de Hecke de $z$, et $\beta_{h}$ comme $\beta_{h}^{\prime} \otimes 1_{B_{h}}$ où $\beta_{h}^{\prime}$ est l'application duale de l'application

$$
\mathcal{C}^{(h)}(\bar{N} \cap H, L)^{m} \widehat{\otimes}_{L} \mathcal{C}^{(h+1)}(\widetilde{M} \cap \widetilde{H}, L) \rightarrow \mathcal{C}^{(h+1)}(\bar{N} \cap H, L)^{m} \widehat{\otimes}_{L} \mathcal{C}^{(h+1)}(\widetilde{M} \cap \widetilde{H}, M)
$$

induite de l'inclusion de $\mathcal{C}^{(h)}(\bar{N} \cap H, L)^{m}$ dans $\mathcal{C}^{(h+1)}(\bar{N} \cap L)^{m}$. Cette dernière étant une application compacte, l'application $\beta_{h}$ est $B_{h}$-compacte. On pose alors $z_{h}=\beta_{h} \circ \alpha_{h}$, qui est $B_{h}$-compacte puisque $\beta_{h}$ l'est. Il est alors facile de vérifier la formule pour $\alpha_{h} \circ \beta_{h}$.

Remarquons à présent que l'action du groupe $M^{0}$ par conjugaison préserve le groupe $H$, ainsi que tous les espaces $\Pi_{\widetilde{H}}^{(h)}$. Ainsi chaque $V_{h}$ est muni d'une action $B_{h}$-linéaire de $M^{0}$. En particulier, chaque espace $V_{h}$ est naturellement un $L\left[M^{0}\right]$-module. Comme le groupe $M^{0} /(M \cap H)$ est fini, chaque $V_{h}$ est un $L\left[M^{0}\right] \otimes_{L[M \cap H]} B_{h}$-module de Banach. Comme $L\left[M^{0}\right] \otimes_{L[M \cap H]} B_{h}$ est une $B_{h}$-algèbre finie étale, le $L\left[M^{0}\right] \otimes_{L[M \cap H]} B_{h}$-module de Banach $V_{h}$ s'identifie donc à un facteur direct du $L\left[M^{0}\right] \otimes_{L[M \cap H]} B_{h}$-module orthonormalisable $L[M] \otimes_{L[M \cap H]} V_{h}$, et vérifie donc la propriété $(\operatorname{Pr})$. Comme $M \cap H$ est un pro-p-groupe uniforme commutatif, il est isomorphe à une certaine puissance du groupe $\mathbb{Z}_{p}$, la transformée d'Amice (cf. [54, Thm. 2.2]) fournit alors un isomorphisme de $L$-algèbre de Banach $B_{h} \cong \Gamma\left(U_{h}^{\prime}, \mathcal{O}_{Y}\right)$ où

$$
Y=\widehat{\widetilde{M} \cap \widetilde{H}}
$$

est l'espace de caractères continus de $(M \cap H) \times \mathbb{Z}_{p}^{q}$ et $U_{h}^{\prime}$ est un certain ouvert affinoïde de $Y$, les $U_{h}^{\prime}$ formant un recouvrement admissible de $Y$. L'algèbre $L[M] \otimes_{L[M \cap H]} B_{h}$ est alors isomorphe à l'anneau structural de l'ouvert affinoïde

$$
U_{h} \subset \mathcal{W}=\widehat{M^{0} \times \mathbb{Z}_{p}^{q}}
$$

image réciproque de $U_{h}^{\prime}$, ce qui achève la preuve.

5.3. Un lemme sur les systèmes de racines de type $A$. Soit $K$ une extension finie de $\mathbb{Q}_{p}$ et $L$ et une extension finie de $\mathbb{Q}_{p}$ telle qu'il existe $\left[K: \mathbb{Q}_{p}\right]$ plongements de $K$ dans $L$.

Le foncteur $H \mapsto L \times_{\mathbb{Q}_{p}} \operatorname{Res}_{K / \mathbb{Q}_{p}}(H)$ de la catégorie des groupes algébriques sur $K$ vers la catégorie des groupes algébriques sur $L$ transforme un groupe déployé sur $K$ en un groupe déployé sur $L$.

Proposition 5.4. Soit $\lambda$ un poids strictement dominant de $L \times_{\mathbb{Q}_{p}} \operatorname{Res}_{K / \mathbb{Q}_{p}}\left(\mathrm{GL}_{n, K}\right)$. On fixe $P_{I} \subset \mathrm{GL}_{n, K}$ un sous-groupe parabolique standard, de sous-groupe de Levi $L_{I}$, et $w$ un

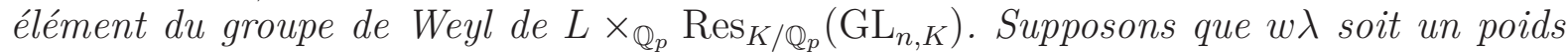
dominant de

$$
L \times_{\mathbb{Q}_{p}} \operatorname{Res}_{K / \mathbb{Q}_{p}}\left(L_{I}\right) \cong \prod_{\tau: K \hookrightarrow L} L_{I, \tau} .
$$

Alors il existe un élément $i \notin I$ tel que

$$
\left\langle w \lambda-\lambda, \tilde{\beta}_{i}\right\rangle \leq-\min _{\tau, i}\left(\lambda_{\tau, i}-\lambda_{\tau, i+1}\right)
$$

où $\tilde{\beta}_{i}$ est la composée

$$
\mathbb{G}_{m, L} \stackrel{\text { diag }}{\longrightarrow} L \times_{\mathbb{Q}_{p}} \operatorname{Res}_{K / \mathbb{Q}_{p}} \mathbb{G}_{m, K} \stackrel{\operatorname{id} \times \operatorname{Res}\left(\beta_{i}\right)}{\longrightarrow} L \times_{\mathbb{Q}_{p}} \operatorname{Res}_{K / \mathbb{Q}_{p}} \mathrm{GL}_{n, K} .
$$


Démonstration. Notons $I=I_{1} \sqcup \cdots \sqcup I_{r}$ la décomposition de $I$ en parties orthogonales. Fixons $\tau$ un plongement de $K$ dans $L$. Comme $w_{\tau} \lambda_{\tau}$ est un poids strictement dominant de $L_{I, \tau}$, on a $\lambda_{w_{\tau}^{-1}\left(i_{1}\right)}>\cdots>\lambda_{w_{\tau}^{-1}\left(i_{s}\right)}$ pour $1 \leq j \leq r$ et $I_{j}=\left\{i_{1}<\cdots<i_{s}\right\}$. Notons $j_{\tau}$ le plus petit $j$ tel que $\left.w_{\tau}^{-1}\right|_{I_{j}}$ ne soit pas l'identité. On a alors nécessairement $\lambda_{\tau, w_{\tau}^{-1}(i)} \leq \lambda_{\tau, i}$ pour $i \in I_{j_{\tau}}$ et il existe $i_{j} \in I_{j_{\tau}}$ tel que $\lambda_{\tau, w_{\tau}^{-1}\left(i_{j}\right)}<\lambda_{\tau, i_{j}}$, ce qui implique alors $\lambda_{\tau, w_{\tau}^{-1}(i)}<\lambda_{\tau, i}$ (et donc $\lambda_{\tau, w_{\tau}^{-1}(i)} \leq \lambda_{\tau, i+1}$ ) pour $i \in I_{j_{\tau}}$ et $i \geq i_{j}$. On a alors

$$
\left\langle w_{\tau} \lambda_{\tau}-\lambda_{\tau}, \beta_{\left|I_{1}\right|+\cdots+\left|I_{j_{\tau}}\right|}\right\rangle=\sum_{i=1}^{\left|I_{j_{\tau}}\right|}\left(\lambda_{w_{\tau}^{-1}(i)}-\lambda_{\tau, i}\right) \leq-\min _{i}\left(\lambda_{\tau, i}-\lambda_{\tau, i+1}\right) .
$$

De plus, on a

$$
\left\langle w_{\tau} \lambda_{\tau}-\lambda_{\tau}, \beta_{\left|I_{1}\right|+\cdots+\left|I_{j^{\prime}}\right|}\right\rangle=0
$$

pour $j^{\prime}<j_{\tau}$. En posant $j=\min _{\tau \in \operatorname{Hom}(K, L)} j_{\tau}$, on vérifie facilement que

$$
\left\langle w \lambda-\lambda, \tilde{\beta}_{j}\right\rangle=\sum_{\tau \in \operatorname{Hom}(K, L)}\left\langle w_{\tau} \lambda_{\tau}-\lambda_{\tau}, \beta_{j}\right\rangle \leq-\min _{\tau, i}\left(\lambda_{\tau, i}-\lambda_{\tau, i+1}\right) .
$$

5.4. Quelques résultats de géométrie rigide. Un espace analytique rigide $X$ est dit quasi-Stein (cf. [40, §2]) si il existe un recouvrement admissible de $X$ par des ouverts affines $U_{1} \subset U_{2} \subset \cdots \cdots U_{n} \subset \cdots$ tel que pour tout $i$, l'image de $\mathcal{O}_{X}\left(U_{i+1}\right)$ dans $\mathcal{O}_{X}\left(U_{i}\right)$ soit dense. On vérifie immédiatement qu'un sous-espace analytique fermé d'un espace quasi-Stein est quasi-Stein.

Lemme 5.5. Soit $\mathcal{M}$ est un faisceau cohérent sur $X$ espace quasi-Stein, muni d'un recouvrement admissible comme ci-dessus. On note $M$ l'espace de ses sections globales que l'on munit de la topologie de la limite projective donnée par la formule $M=\lim _{i} \Gamma\left(U_{i}, \mathcal{M}\right)$. Alors pour tout ouvert admissible $U$ de $X$, l'application

$$
\mathcal{O}_{X}(U) \widehat{\otimes}_{\mathcal{O}(X)} M \rightarrow \Gamma(U, \mathcal{M})
$$

est un isomorphisme topologique.

Démonstration. L'algèbre $\mathcal{O}_{X}(X)$ est une algèbre de Fréchet-Stein, ainsi [55, Cor. 3.1] montre que, pour tout $i$, on a $\Gamma\left(U_{i}, \mathcal{M}\right) \simeq \mathcal{O}_{X}\left(U_{i}\right) \otimes_{\mathcal{O}(X)} M$. Soit $V$ un ouvert affinoïde de $X$. Par admissibilité du recouvrement, il existe $i$ tel que $V \subset U_{i}$, ainsi on en déduit que

$$
\Gamma(V, \mathcal{M}) \simeq \mathcal{O}_{X}(V) \otimes_{\mathcal{O}_{X}\left(U_{i}\right)} \Gamma\left(U_{i}, \mathcal{M}\right) \simeq \mathcal{O}_{X}(V) \otimes_{\mathcal{O}(X)} M .
$$

En utilisant, [9, Prop. 9.1.4.2.(i)], $U$ possède un recouvrement admissible par des ouverts affinoïdes. On conclut en utilisant la formule $\Gamma(U, \mathcal{M}) \simeq \lim _{V \subset U} \Gamma(V, \mathcal{M})$ la limite projective étant prise sur tous les ouverts affinoïdes contenus dans $U$.

Lemme 5.6. Soit $f: X \rightarrow Y$ un morphisme d'espaces analytiques quasi-Stein. Si $V$ est un ouvert admissible de $Y$, alors

$$
\mathcal{O}_{X}\left(f^{-1}(V)\right) \simeq \mathcal{O}(X) \widehat{\otimes}_{\mathcal{O}(Y)} \mathcal{O}_{Y}(V) .
$$

Démonstration. On fixe $\left(U_{i}\right)_{i \geq 1}$ et $\left(V_{i}\right)_{i \geq 1}$ des recouvrements admissibles de $X$ et $Y$ vérifiant la propriété ci-dessus. Par admissibilité du recouvrement $\left(V_{i}\right)_{i \geq 1}$, on peut, quitte à en extraire une sous-suite, supposer que pour tout $i \geq 1$, on a $f\left(U_{i}\right) \subset V_{i}$. Soit $f_{i}: U_{i} \rightarrow V_{i}$ le morphisme induit. La propriété de faisceau nous donne un isomorphisme 
$\mathcal{O}_{X}\left(f^{-1}(V)\right) \simeq \lim _{i} \mathcal{O}_{X}\left(f_{i}^{-1}\left(V \cap V_{i}\right)\right)$. On peut donc supposer $X$ et $Y$ affinoïdes. On utilise alors la propriété de faisceau pour écrire $\mathcal{O}_{X}\left(f^{-1}(V)\right) \simeq \lim _{U \subset V} \mathcal{O}_{X}\left(f^{-1}(U)\right)$, où la limite projective est prise sur les ouverts affinoïde contenus dans $V$. Lorsque $X, Y$ et $U$ sont affinoïdes, on a alors $\mathcal{O}_{X}\left(f^{-1}(U)\right) \simeq \mathcal{O}(X) \widehat{\otimes}_{\mathcal{O}(Y)} \mathcal{O}_{Y}(U)$.

Lemme 5.7. Soit $X, Y$ et $Z$ trois espaces rigides analytiques lisses, ainsi que $f: X \rightarrow Y$, $g: Y \rightarrow Z$ et $h=g \circ f$ des applications rigides analytiques. Si $x \in X$, que $h$ est lisse au point $x$ et $g$ lisse au point $f(x)$. Alors l'application $f$ est lisse au point $x$ si et seulement si l'application $f_{h(x)}$ de $X \otimes k(h(x))$ dans $Y \otimes k(h(x))$ est lisse au point $x$.

Démonstration. Il suffit de prouver que si $f_{h(x)}$ est lisse en $x$ alors il en est de même de $x$, l'implication inverse résultat de la préservation de la lissité par changement de base. Supposons donc $f_{h(x)}$ lisse. Il suffit de prouver que l'application $d f_{x}$ sur les plans tangents $T_{x} X \rightarrow T_{f(x)} Y$ est surjective. Dans le diagramme commutatif ci-dessous, les lignes sont exactes, ce qui résulte de la lissité de $g$ et $h$.

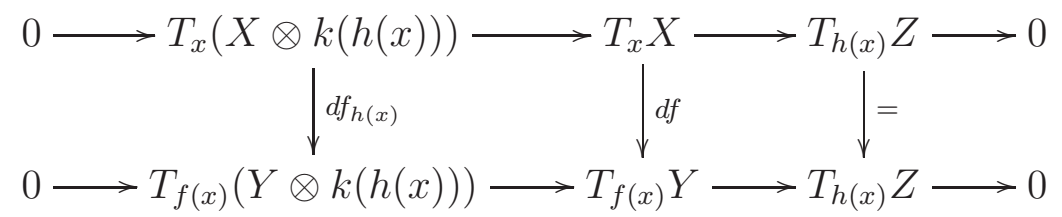

La lissité de $d f_{h(x)}$ implique alors la surjectivité de la flèche verticale de gauche. Comme la flèche verticale de droite est l'identité, le lemme des cinq implique que la flèche verticale du milieu est surjective.

\subsection{Descente de la lissité en géométrie rigide.}

Lemme 5.8. Soit $X, Y, Z$ trois espaces rigides analytiques sur $\mathbb{Q}_{p}$, ainsi que $f: X \rightarrow Y$ et $g: Y \rightarrow Z$ des applications rigides analytiques. Posons $h=g \circ f$. Soit $x \in X, y=f(x)$ et $z=g(y)$. Si $f$ est plat et $h$ est lisse en $x$, alors $g$ est lisse en $y$.

Démonstration. D'après [1, Prop. 5.10.3.(ii)], le morphisme $g$ est plat en $y$. D'après [1, Prop. 6.4.22], il suffit de prouver que $Y \otimes_{Z} k(z)$ est lisse en $y$. On est donc ramené à considérer la situation suivante : $Z=\operatorname{Sp}(k)$ pour $k$ une extension finie de $\mathbb{Q}_{p}, X$ et $Y$ sont deux espaces rigides sur $k$ et $f: X \rightarrow Y$ est un morphisme plat en $x \in X$. Il suffit de prouver que si $X$ est $k$-lisse en $x, Y$ est $k$-lisse en $y=f(x)$. Soit $\mathcal{O}$ l'anneau des entiers de $k$. L'assertion étant locale en $x$, on peut supposer que $X=\operatorname{Spf}(B)^{\text {rig }}$ et $Y=\operatorname{Sp}(A)^{\text {rig }}$ avec $A$ et $B$ deux $\mathcal{O}$-algèbres topologiquement de type fini sur $\mathcal{O}$. On note $\mathfrak{p}_{y} \subset A$ et $\mathfrak{p}_{x} \subset B$ les idéaux premiers correspondants aux points $y$ et $x$. D'après [1, Prop. 6.4.12], $X$ est lisse en $x$ si et seulement si l'anneau local $B_{\mathfrak{p}_{x}}$ est une $k$-algèbre formellement lisse ([1, Prop. 1.14.5]). Comme $k$ est de caractéristique 0, l'anneau local $B_{\mathfrak{p}_{x}}$ est une $k$-algèbre formellement lisse si et seulement si c'est un anneau régulier ([31, Prop. Thm. 19.6.4]). Comme de plus $f$ est plat en $x$, on déduit de [1, Prop. 5.5.1] que l'anneau $B_{\mathfrak{p}_{x}}$ est une $A_{\mathfrak{p}_{y}}$-algèbre plate. Ainsi si $X$ est $k$-lisse en $x$, l'anneau $B_{\mathfrak{p}_{x}}$ est régulier et la Proposition $[31,17.3 .3 .(\mathrm{i})]$ permet alors de conclure que $A_{\mathfrak{p}_{y}}$ est un anneau local régulier. On déduit alors comme précédemment que $Y$ est lisse sur $k$ en $f(x)$.

\section{RÉFÉRENCES}

[1] A. Abbes - «Éléments de géométrie rigide», Progress in Mathematics, 286. Springer Basel AG 2010, 477 p., 2010. 
[2] P. Allen - « Deformations of polarized automorphic Galois representations and adjoint Selmer groups, arXiv : 1411.7661.

[3] T. Barnet-Lamb, T. Gee, D. Geraghty \& R. Taylor - « Potential automorphy and change of weight », Annals of Math. (à paraître).

[4] J. Bellaïche \& G. Chenevier - « Families of Galois representations and Selmer groups », Astérisque (2009), no. 324, p. xii+314.

[5] J. Bergdall - « Paraboline variation over $p$-adic families of $(\varphi, \Gamma)$-modules, arXiv :1410.3412v1.

[6] L. Berger - «Représentations p-adiques et équations différentielles », Invent. Math. 148 (2002), no. 2, p. 219-284.

[7] L. Berger \& P. Colmez - « Familles de représentations de de Rham et monodromie p-adique », Astérisque (2008), no. , p. 303-337.

[8] P. Berthelot - « Cohomologie rigide et cohomologie rigide à supports propres ».

[9] S. Bosch, U. Güntzer \& R. Remmert - « Non-Archimedean analysis. A systematic approach to rigid analytic geometry. », Grundlehren der Mathematischen Wissenschaften, 261. Berlin etc. : Springer Verlag. XII, 436 p. DM 168.00 (1984)., 1984.

[10] C. BreuiL - « Vers le socle localement analytique pour $\mathrm{GL}_{n} »$.

[11] _ _ « Vers le socle localement analytique pour $\mathrm{GL}_{n}$ II », Math. Annalen (à paraître).

[12] K. BuzzARD - «Eigenvarieties. », in L-functions and Galois representations. Based on the symposium, Durham, UK, July 19-30, 2004, Cambridge : Cambridge University Press, 2007, p. 59-120 (English).

[13] A. Caraiani - « Local-global compatibility and the action of monodromy on nearby cycles », Duke Math. J. 161 (2012), no. 12, p. 2311-2413.

[14] _ « Monodromy and local-global compatibility for $\mathrm{l}=\mathrm{p}$ », Algebra Number Theory 8 (2014), no. 7, p. 1597-1646.

[15] A. Caraiani, M. Emerton, T. Gee, D. Geraghty, V. Paškūnas \& S. W. Shin - « Patching and the $p$-adic local langlands correspondence », arXiv :1310.0831.

[16] G. Chenevier - « Familles p-adiques de formes automorphes pour $\mathrm{GL}_{n}$. », J. Reine Angew. Math. 570 (2004), p. 143-217 (French).

[17] _ « Une correspondance de Jacquet-Langlands p-adique. », Duke Math. J. 126 (2005), no. 1, p. 161-194 (French).

[18] _ « On the infinite fern of Galois representations of unitary type », Ann. Sci. Éc. Norm. Supér. (4) 44 (2011), no. 6, p. 963-1019.

[19] — « Sur la densité des représentations cristallines de $\operatorname{Gal}\left(\overline{\mathbb{Q}}_{p} / \mathbb{Q}_{p}\right)$ », Math. Ann. 355 (2013), no. 4, p. 1469-1525.

[20] G. Chenevier \& M. Harris - « Construction of automorphic Galois representations. II. », Camb. J. Math. 1 (2013), no. 1, p. 53-73 (English).

[21] L. Clozel, M. Harris, R. TAylor - «Automorphy for some $l$-adic lifts of automorphic mod $l$ Galois representations », Pub. Math. I.H.É.S. 108 (2008), p. 1-181.

[22] P. Colmez - «Représentations triangulines de dimension $2 »$, Astérisque (2008), no. 319, p. 213258.

[23] P. Colmez \& G. Dospinescu - « Complétés universels de représentations de $\mathrm{GL}_{2}\left(\mathbb{Q}_{p}\right)$ », Algebra and Number Theory, à paraître.

[24] B. Conrad - «Irreducible components of rigid spaces. », Ann. Inst. Fourier 49 (1999), no. 2, p. 473-541 (English).

[25] M. Emerton - « Locally analytic vectors in representations of locally p-adic analytic groups », (2004), A paraître à Memoir of the AMS.

[26] _ - « Jacquet modules of locally analytic representations of $p$-adic reductive groups. I. Construction and first properties », Ann. Sci. École Norm. Sup. (4) 39 (2006), no. 5, p. 775-839.

[27] — - "On the interpolation of systems of eigenvalues attached to automorphic Hecke eigenforms », Invent. Math. 164 (2006), no. 1, p. 1-84. 
[28] _ - «acquet modules of locally analytic representations of $p$-adic reductive groups II. The relation to parabolic induction », (2007), à paraître à J. Inst. Math. de Jussieu.

[29] M. Emerton \& T. GeE - « A geometric perspective on the Breuil-Mézard conjecture », à paraître à J. Inst. Math. de Jussieu.

[30] J.-M. Fontaine - «Représentations -adiques potentiellement semi-stables », Astérisque (1994), no. 223 , p. $321-347$.

[31] A. Grothendieck - «Éléments de géométrie algébrique. IV. Étude locale des schémas et des morphismes de schémas. I», Pub. Math. I.H.É.S. (1964), no. 20, p. 259.

[32] D. HAnsen - « Universal eigenvarieties, trianguline Galois representations, and $p$-adic Langlands functoriality J. reine angew. Math., à paraître.

[33] E. Hellmann - « Families of trianguline representations and finite slope spaces », arXiv :1202.4408.

[34] « « Families of $p$-adic Galois representations and $(\varphi, \Gamma)$-modules », arXiv :1202.3413.

[35] E. Hellmann \& B. Schraen - «Density of potentially crystalline representations of fixed weight », arXiv :1311.3249.

[36] R. Huber - «éEtale cohomology of rigid analytic varieties and adic spaces, Aspects of Math., Springer.

[37] J. E. Humphreys - Representations of semisimple Lie algebras in the BGG category $\mathcal{O}$, Graduate Studies in Mathematics, vol. 94, American Mathematical Society, Providence, RI, 2008.

[38] A. J. DE Jong - « Crystalline Dieudonné module theory via formal and rigid geometry », Publ. Math. I.H.É.S. (1995), no. 82, p. 5-96 (1996).

[39] K. Kedlaya, J. Pottharst \& L. XiaO - « Cohomology of arithmetic families of $(\phi, \Gamma)$-modules », J. Amer. Math. Soc. 27 (2014), 1043-1115.

[40] R. KIEHL - « Theorem A und Theorem B in der nichtarchimedischen Funktionentheorie », Invent. Math. 2 (1967), p. 256-273.

[41] M. Kisin - « Overconvergent modular forms and the Fontaine-Mazur conjecture », Invent. Math. 153 (2003), no. 2, p. 373-454.

[42] _ , «Potentially semi-stable deformation rings », J. Amer. Math. Soc. 21 (2008), no. 2, p. 513546.

[43] _ « Moduli of finite flat group schemes, and modularity », Ann. of Math. (2) 170 (2009), no. 3 , p. $1085-1180$.

[44] S. S. Kudla - « The local Langlands correspondence : the non-Archimedean case », in Motives (Seattle, WA, 1991), Proc. Sympos. Pure Math., vol. 55, Amer. Math. Soc., Providence, RI, 1994, p. $365-391$.

[45] J.-P. LABESSE - « Changement de base CM et séries discrètes », in On the stabilization of the trace formula, Stab. Trace Formula Shimura Var. Arith. Appl., vol. 1, Int. Press, Somerville, MA, 2011, p. $429-470$.

[46] M. Lazard - «Groupes analytiques p-adiques », Pub. Math. I.H.É.S. 26 (1965), p.5-219.

[47] R. Liu - « Cohomology and duality for $(\phi, \Gamma)$-modules over the Robba ring » Int. Math. Res. Not. (2008).

[48] R. LIU - « Triangulation of refined families », arXiv :1202.2188.

[49] S. Orlik \& M. Strauch - « On the irreducibility of locally analytic principal series representations. », à paraître dans Representation Theory, arXiv :math/0612809v2, 2006.

[50] _ _ «On Jordan-Hölder Series of some Locally Analytic Representations », J. Amer. Math. Soc. 28 (2015), 99-157.

[51] P. SchneIder - Nonarchimedean functional analysis, Springer Monographs in Mathematics, Springer Verlag, 2002.

[52] P. Schneider \& J. Teitelbaum - « Banach space representations and Iwasawa theory », Israel J. Math. 127 (2002), p. 359-380.

[53] _ « Locally analytic distributions and $p$-adic representation theory, with applications to $\mathrm{GL}_{2}$ », J. Amer. Math. Soc. 15 (2001), p. 443-468. 
[54] _ «

[55] _ « Algebras of $p$-adic distributions and admissible representations », Invent. Math. 153 (2003), p. 145-196.

[56] _ « Banach-Hecke algebras and p-adic Galois representations », Doc. Math. (2006), no. Extra Vol., p. 631-684.

[57] _ « ¿ Duality for admissible locally analytic representations », Represent. Theory 9 (2005), p. 297-326.

[58] B. Schraen - «Représentations localement analytiques de $\mathrm{GL}_{3}\left(\mathbb{Q}_{p}\right) »$, Ann. Sci. Éc. Norm. Supér. 44 (2011), p. 43-145.

[59] J.-P. SERRE - « Cohomologie galoisienne », Lecture Notes in Mathematics, 5. Springer-Verlag, Berlin, 1994.

[60] J. Thorne - « On the automorphy of $\ell$-adic Galois representations with small residual image », $J$. Institut Math. Jussieu 11 (2012), p. 855-920.

[61] A. V. Zelevinsky - «Induced representations of reductive p-adic groups. II. On irreducible representations of GL(n)», Ann. Sci. École Norm. Sup. (4) 13 (1980), no. 2, p. 165-210.

Christophe Breuil, C.N.R.S., Université Paris-Sud, Faculté D’Orsay, BÂtiment 425, F91405 Orsay Cedex, France, Christophe.Breuil@math.u-Psud.fr

Eugen Hellmann, Mathematisches Institut, Universität Bonn, Endenicher Allee 60, D-53115 Bonn, Germany, hellmann@math.uni-Bonn.De

Benjamin Schraen, C.N.R.S., Laboratoire de Mathématique, Université de Versailles St. Quentin, 45 Avenue des États-Unis, F-78035 Versailles, France, Benjamin.schraen@uvsq.Fr 